University of Tennessee Health Science Center UTHSC Digital Commons

\title{
Biochemical and Structural Characterization of the Atg8/LC3 Lipidation Pathway
}

\author{
Yumei Zheng \\ University of Tennessee Health Science Center
}

Follow this and additional works at: https://dc.uthsc.edu/dissertations

Part of the Medical Sciences Commons

\section{Recommended Citation}

Zheng, Yumei (https://orcid.org/0000-0002-4033-2670), "Biochemical and Structural Characterization of the Atg8/LC3 Lipidation Pathway" (2020). Theses and Dissertations (ETD). Paper 514. http://dx.doi.org/ 10.21007/etd.cghs.2020.0499.

This Dissertation is brought to you for free and open access by the College of Graduate Health Sciences at UTHSC Digital Commons. It has been accepted for inclusion in Theses and Dissertations (ETD) by an authorized administrator of UTHSC Digital Commons. For more information, please contact jwelch30@uthsc.edu. 


\title{
Biochemical and Structural Characterization of the Atg8/LC3 Lipidation Pathway
}

\begin{abstract}
Atg8 and its eukaryotic orthologues LC3 and GARBARAP family proteins (referred here to Atg8 family proteins) play crucial roles in autophagy through their covalent ligation to lipids, typically phosphatidylethanolamine (PE), in a process known as lipidation. Lipidation of Atg8 family proteins regulates numerous facets of the autophagy process, including regulating expansion of the phagophore membrane, recruiting selected cargoes for degradation, and providing an autophagosome membranebound platform mediating dynamic interactions with other regulatory proteins. Atg8 family proteins are ubiquitin-like proteins (UBLs), and their lipidation involves a divergent UBL conjugation cascade including Atg7, Atg3, and Atg12-Atg5-Atg16 acting as E1, E2, and E3 enzymes, respectively. Atg7 initiates Atg8 conjugation by catalyzing their C-terminal adenylation and conjugation to the catalytic cysteine of Atg3. Ultimately, the Atg12-Atg5-Atg16 complex catalyzes Atg8 ligation to a primary amino group on PE or other acceptor lipids. Molecular mechanisms underlying Atg8 lipidation remain poorly understood despite association of Atg3, the E1 Atg7, and the composite E3 Atg12-Atg5-Atg16 with pathologies including cancers, infections, and neurodegeneration. The first part of this dissertation work describes methods for expressing and purifying human LC3 or GABARAP, ATG7, ATG3, and the ATG12-ATG5-ATG16L1 complex for in vitro studies of LC3/GABARAP lipidation; based on these protocol established, we report that an Atg3 element we term E123IR (E1, E2, and E3-interacting region) is an allosteric switch, by studying yeast enzymes. Nuclear magnetic resonance (NMR), biochemical, crystallographic and genetic data collectively indicate that in the absence of the enzymatic cascade, the Atg3E123IR makes intramolecular interactions restraining Atg3's catalytic loop, while E1 and E3 enzymes directly remove this brace to conformationally activate Atg3 and elicit Atg8 lipidation in vitro and in vivo. We propose that Atg3's E123IR protects the E2 UBL thioester bond from wayward reactivity toward errant nucleophiles, while Atg8 lipidation cascad
\end{abstract}

Document Type

Dissertation

Degree Name

Doctor of Philosophy (PhD)

Program

Biomedical Sciences

Research Advisor

Brenda A. Schulman, PhD

Keywords

Atg8, autophagy, E2, ligase, lipidation, ubiquitin-like protein

Subject Categories

Medical Sciences | Medicine and Health Sciences 


\section{Biochemical and Structural Characterization of the Atg8/LC3 Lipidation Pathway}

Author:

Yumei Zheng
Advisor:

Brenda A. Schulman, PhD

A Dissertation Presented for The Graduate Studies Council of

The University of Tennessee Health Science Center

in Partial Fulfillment of the Requirements for the

Doctor of Philosophy degree from

The University of Tennessee

in

Biomedical Sciences: Microbiology, Immunology, and Biochemistry College of Graduate Health Sciences 
Chapter 2 (c) 2017 Elsevier Inc.

All other materials (C) 2020 Yumei Zheng. All rights reserved. 


\section{DEDICATION}

I dedicate my dissertation to my parents Guoqing Zheng and Wei Liu, my loving grandparents, and my incredible husband Dr. Xi-Zhi (Johnny) Guo. I am deeply indebted to their unconditional and endless love, care and supports that have guarded me through not only this rocky journey pursuing a doctoral degree in a foreign country, but also through my entire life.

I dedicate this work to my son Elliott Guo, who has been a surprising gift and the greatest bless. He has empowered me to always stay calm, strong-minded and optimistic facing up unexpected difficulties in science and personal life.

I dedicate this work to my $\mathrm{PhD}$ advisor Dr. Brenda A. Schulman for being the best mentor possible. I am grateful to her tremendous patience and efforts guiding me into the mansion of science, as well as to her passionate and generous personalities that inspire me to always keep my decency both in science and in personal life.

I also dedicate this work to my sweet cat Earl, my affectionate cousin Dr. Chen $\mathrm{Xu}$, my childhood friend Wan-Ting Dong, and my closest colleague Dr. Yu Qiu. I sincerely appreciate their constant supports and warm companionship. 


\section{ACKNOWLEDGEMENTS}

I would like to thank the rest of my dissertation committee members Dr. Charles Rock, Dr. Eric Enemark, Dr. Mondira Kundu and Dr. Julio Cordero-Morales for their great mentorship and invaluable advice. I am grateful to all (previous) Schulman lab members, especially Dr. Danny Scott, Dr. Kuen-Phon Wu, Dr. Nicolas Brown, Dr. Ryan VanderLinden, Dr. Randy Watson, Shein Ei Cho, Shelia Bozeman and David Miller, for their generous helps inside and outside the lab. I am thankful to Dr. Xu Liu and Dr. Daniel Klionsky at University of Michigan, for their crucial experimental data and remarks that composes an essential part of my final dissertation. I am appreciative of Dr. Grace Royappa and Dr. Darcie Miller for their professional technical facilitations. I show gratitude for Dr. Ryan Potts and his lab members for their scientific and mental supports. I thank my friends Dr. Yinan Gong, Dr. Qifan Zhu, Dr. Qianxia (Sherry) Zhang, Dr. Cheng Tian, Qiyue (Ceci) Chen, Xin Lan and Hengyu Jiang, and Thomas lab members for their companionship. 


\begin{abstract}
Atg8 and its eukaryotic orthologues LC3 and GARBARAP family proteins (referred here to Atg8 family proteins) play crucial roles in autophagy through their covalent ligation to lipids, typically phosphatidylethanolamine (PE), in a process known as lipidation. Lipidation of Atg8 family proteins regulates numerous facets of the autophagy process, including regulating expansion of the phagophore membrane, recruiting selected cargoes for degradation, and providing an autophagosome membranebound platform mediating dynamic interactions with other regulatory proteins. Atg8 family proteins are ubiquitin-like proteins (UBLs), and their lipidation involves a divergent UBL conjugation cascade including Atg7, Atg3, and Atg12-Atg5-Atg16 acting as E1, E2, and E3 enzymes, respectively. Atg7 initiates Atg8 conjugation by catalyzing their C-terminal adenylation and conjugation to the catalytic cysteine of Atg3. Ultimately, the Atg12-Atg5-Atg16 complex catalyzes Atg8 ligation to a primary amino group on PE or other acceptor lipids.

Molecular mechanisms underlying Atg8 lipidation remain poorly understood despite association of Atg3, the E1 Atg7, and the composite E3 Atg12-Atg5-Atg16 with pathologies including cancers, infections, and neurodegeneration. The first part of this dissertation work describes methods for expressing and purifying human LC3 or GABARAP, ATG7, ATG3, and the ATG12-ATG5-ATG16L1 complex for in vitro studies of LC3/GABARAP lipidation; based on these protocol established, we report that an Atg3 element we term E123IR (E1, E2, and E3-interacting region) is an allosteric switch, by studying yeast enzymes. Nuclear magnetic resonance (NMR), biochemical, crystallographic and genetic data collectively indicate that in the absence of the enzymatic cascade, the Atg $3{ }^{\text {E123IR }}$ makes intramolecular interactions restraining Atg3's catalytic loop, while E1 and E3 enzymes directly remove this brace to conformationally activate Atg3 and elicit Atg8 lipidation in vitro and in vivo. We propose that Atg3's E123IR protects the E2 UBL thioester bond from wayward reactivity toward errant nucleophiles, while Atg8 lipidation cascade enzymes induce E2 active site remodeling through an unprecedented mechanism to drive autophagy.
\end{abstract}




\section{TABLE OF CONTENTS}

CHAPTER 1. INTRODUCTION ......................................................................................1

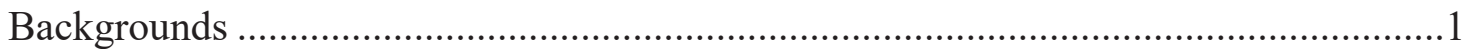

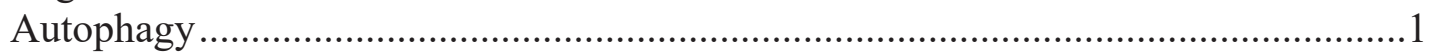

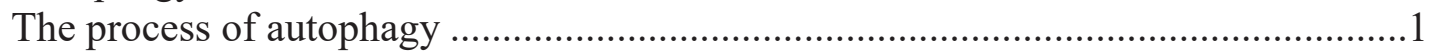

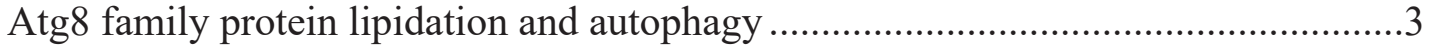

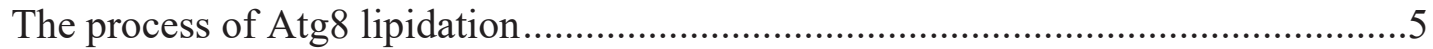

Atg8 family protein lipidation cascade and diseases .........................................

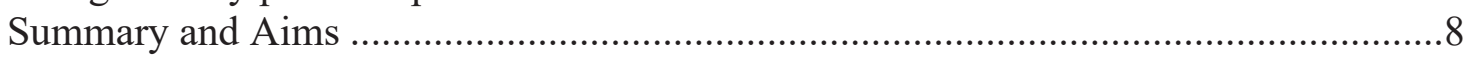

\section{CHAPTER 2. PRODUCTION OF HUMAN ATG PROTEINS FOR}

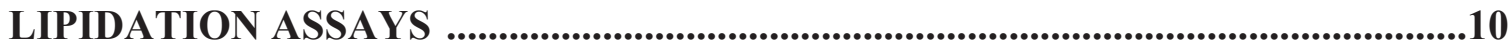

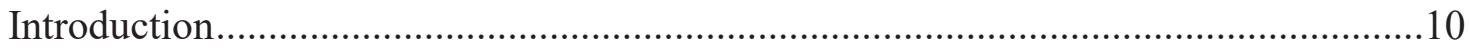

Expression of Human LC3B, ATG7, ATG3, ATG12-ATG5, and ATG16L1 ............11

General overview of protein expression and purification ....................................... 11

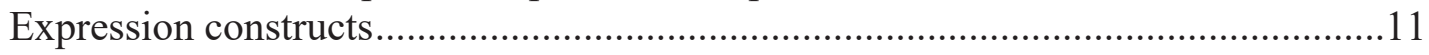

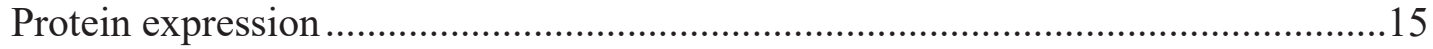

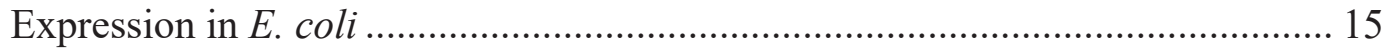

Expression in insect cells ....................................................................... 15

Protein Purification ......................................................................................... 16

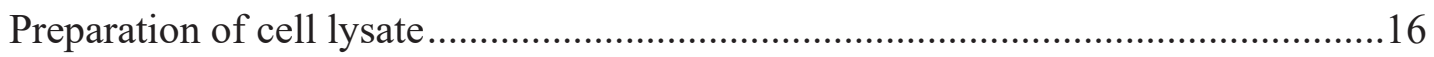

Glutathione-affinity chromatography for LC3B, ATG7, ATG3, and ATG12-

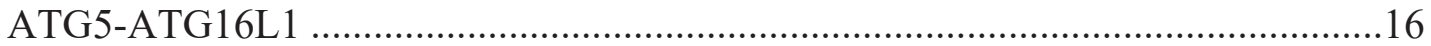

Protease-mediated elution from beads for purification of LC3B, ATG3, and

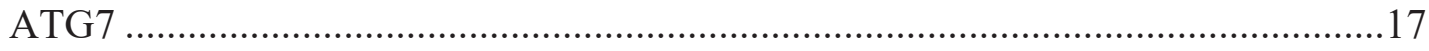

Glutathione-mediated ATG12-ATG5-ATG16L1 elution from GS4B.....................21

Nickel-affinity chromatography, imidazole-mediated elution from nickel beads, and TEV cleavage to obtain ATG12 (52-140)-ATG5-ATG16L1(1-69) complex ...21 Ion exchange chromatography on ATG3 and ATG12(52-140) ATG5-ATG16L1

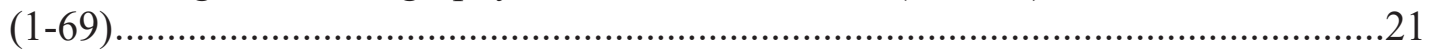

Gel filtration chromatography for LC3B, ATG7, ATG3, and ATG12-ATG5-

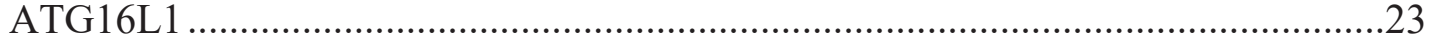

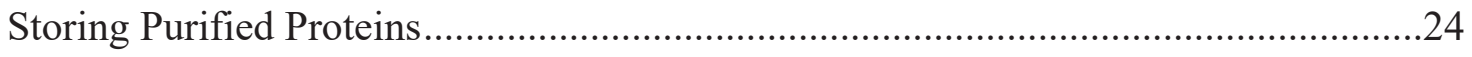

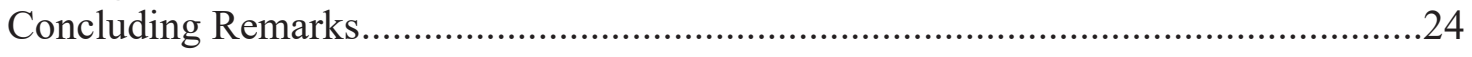

CHAPTER 3. A SWITCH ELEMENT IN THE AUTOPHAGY E2 ATG3 MEDIATES ALLOSTERIC REGULATION ACROSS THE LIPIDATION

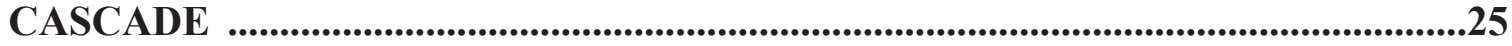

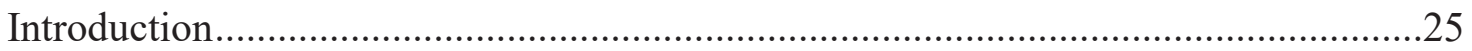

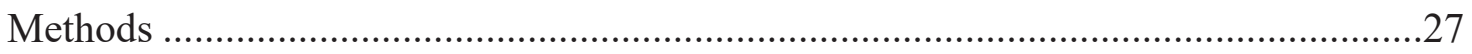

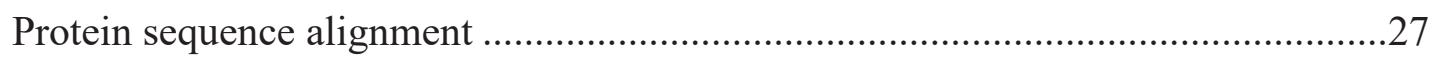

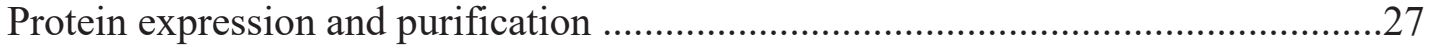

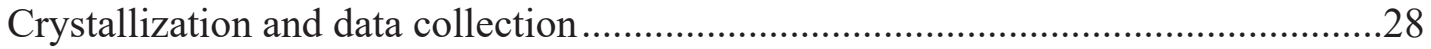

Data collection, processing, and structure refinement .......................................28 


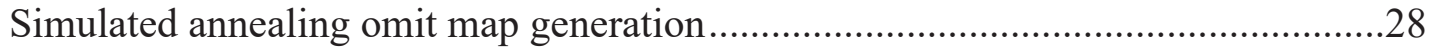

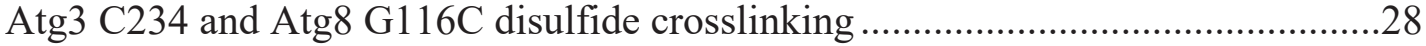

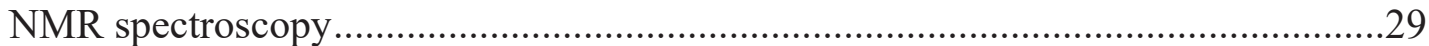

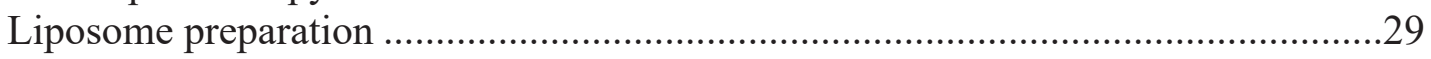

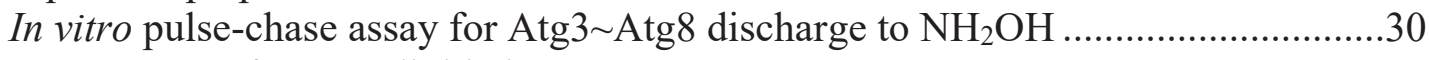

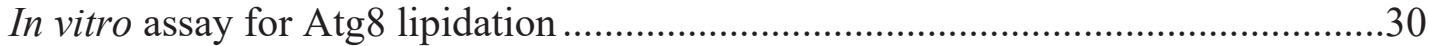

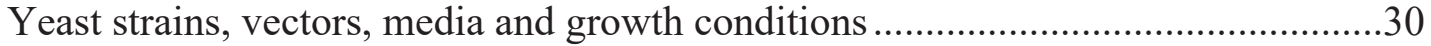

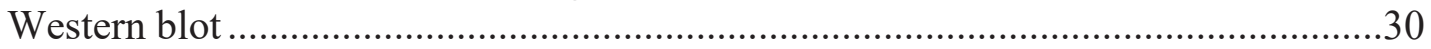

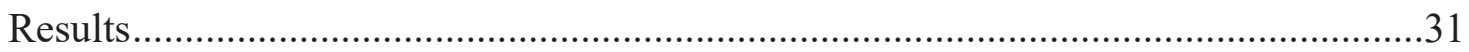

Autophagy E3 activates intrinsic reactivity of Atg3 Atg8 ................................. 31

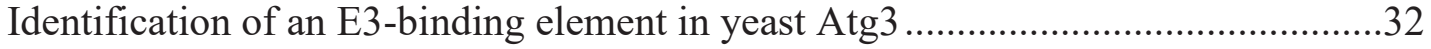

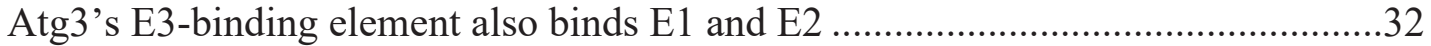

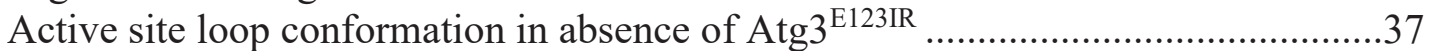

Mutants displacing Atg $3^{\mathrm{E} 123 \mathrm{IR}}$ from Atg $3^{\text {cat }}$ activate ligation ...............................42

Extensive surfaces are required for Atg3 Atg8 activation ..................................44

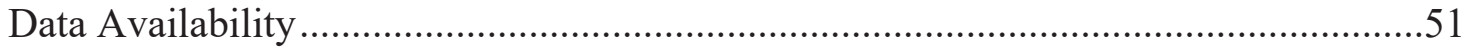

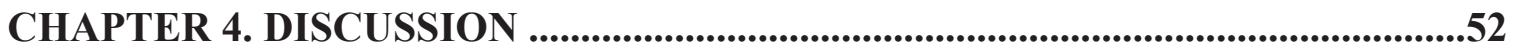

LIST OF REFERENCES ..................................................................................57

VITA 


\section{LIST OF TABLES}

Table 2-1. Plasmids used to produce human ATG proteins for lipidation assays..........13

Table 3-1. Data collection and refinement statistics ................................................41 


\section{LIST OF FIGURES}

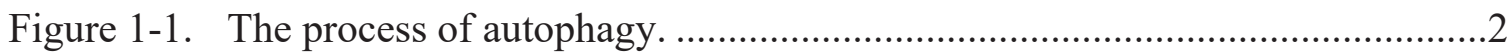

Figure 1-2. Structure comparison of Atg8 and LC3 to other ubiquitin like proteins. .......4

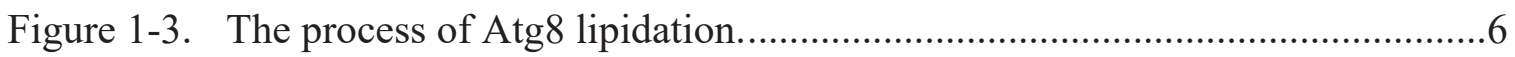

Figure 2-1. Flow-chart indicating the expression system and purification strategies for production of human ATG proteins for lipidation assays. ....................12

Figure 2-2. Graphical representation of plasmids used in this study...........................14

Figure 2-3. 15\% SDS-PAGE showing LC3B (1-120) at different steps of

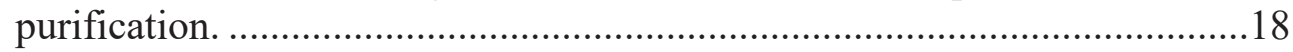

Figure 2-4. 15\% SDS-PAGE showing ATG7 at different steps of purification.............19

Figure 2-5. 15\% SDS-PAGE showing ATG3 at different steps of purification..............20

Figure 2-6. 15\% SDS-PAGE showing ATG12 (52-140)-ATG5-ATG16L1 (1-69)

complex at different stages of purification.............................................22

Figure 3-1. The Atg12-Atg5 module within the autophagy E3 activates intrinsic reactivity of the $A \operatorname{tg} 3 \sim A \operatorname{tg} 8$ intermediate. .............................................26

Figure 3-2. Lack of sequence conservation for Atg3 FR yet functional conservation of FR-binding site on Atg12 within Atg12-Atg5 E3 module....................33

Figure 3-3. E123IR plays essential role in E3-dependent Atg8 ligation activity............34

Figure 3-4. Alanine scan within FR of Atg3 for E3-dependent activation of Atg3 Atg8 intermediate. 35

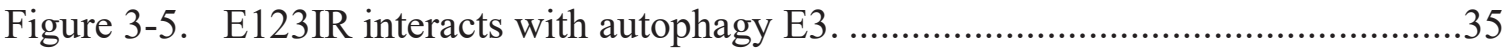

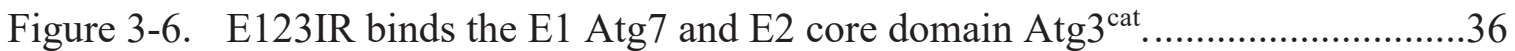

Figure 3-7. Mutations in E123IR hydrophobic residues significantly impair E123IR-

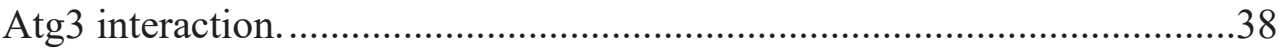

Figure 3-8. Conformational changes upon E123IR removal from Atg3 .......................39

Figure 3-9. Structural remodeling of the Atg3 catalytic core upon displacement or removal of the E123IR element.

Figure 3-10. Mutations in E123IR-binding residues activate Atg3 Atg8 in the absence of $\mathrm{E} 3$ in vitro and in vivo. 
Figure 3-11. Mutations in interface between Atg3's E123IR element and catalytic domain activate the $A \operatorname{tg} 3 \sim \operatorname{Atg} 8$ intermediate.

Figure 3-12. Alanine scan within Atg3 cat and $\mathrm{Atg} 8$ by $\mathrm{NH}_{2} \mathrm{OH}$ discharge assays..........46

Figure 3-13. Extensive surfaces of Atg8 and the Atg3 catalytic domain are required for activation of the Atg3 Atg8 intermediate.

Figure 3-14. Alanine mutants within Atg3cat and Atg8 examined by Atg8-lipidation assays in vitro and in vivo.

Figure 3-15. Modeling of the active conformation of Atg3 Atg8 intermediate. ..............50

Figure 4-1. Schematic model for allosteric regulation of Atg3 activity through E123IR interactions across the lipidation cascade. 


\section{LIST OF ABBREVIATIONS}

AD

ATG

BME

CSP

CTD

DTT

E123IR

ER

FPLC

FR

GABARAP

GST

HECT

HEPES

HR

LC3B

MES

NMR

NTD

PAGE

PEG

RBR

RING

RNF4

SDS

SNARE

STDEV

SUMO

TEV
Adenylation domain

Autophagy related gene

Beta-mercaptoethanol

Chemical shift perturbation

C-terminal domain

Dithiothreitol

$\mathrm{E} 1, \mathrm{E} 2$ and E3 interactive region

Endoplasmic reticulum

Fast protein liquid chromatography

Flexible region

Gamma-aminobutyric acid receptor-associated protein

Glutathione S-transferase

Homologous to the E6-AP Carboxyl Terminus

4-(2-hydroxyethyl)-1-piperazineethanesulfonic acid

Handle region

Microtubule-associated proteins 1A/1B light chain 3B

2-(N-morpholino)ethanesulfonic acid

Nuclear magnet resonance

N-terminal domain

Polyacrylamide

Polyethylene glycol

RING between RING

Really interesting gene

RING finger protein 4

Sodium dodecyl sulfide

N-ethylmaleimide-sensitive factor attachment protein receptor

Standard deviation

Small ubiquitin-like modifier

Tobacco Etch Virus nuclear-inclusion-a endopeptidase 
TRIS

UBE2D1

UBL

UDF

ULK trisaminomethane

Ubiquitin-conjugation enzyme E2 D1

Ubiquitin-like protein

Ubiquitin-fold domain

Unc-51-like kinase 


\section{CHAPTER 1. INTRODUCTION}

\section{Backgrounds}

\section{Autophagy}

Autophagy is a major catabolic pathway by which eukaryotic cells degrade and recycle diverse cellular materials (Yang and Klionsky, 2010). Sectors of the cytosol are first sequestered within a double-membrane-bound autophagosome, and then degraded upon autophagosome fusion with the lysosome in higher eukaryotes or vacuole in yeast (Nakatogawa et al., 2009). Cargoes directed to autophagosomes for degradation include protein assemblies, organelles, misfolded macromolecules, debris, and pathogenic bacteria (Galluzzi et al., 2017; Gatica et al., 2018; Stolz et al., 2014) (Figure 1-1). Autophagy plays important roles in cellular responses upon various forms of stress including starvation, toxicities and pathogenic infections. It is an indispensable cell activity in context of homeostasis, cellular differentiation and development. Dysregulation of autophagy is associated with metabolic, neurologic, and oncologic disorders (Amaravadi et al., 2016; Dikic and Elazar, 2018; Levy et al., 2017; Mizushima et al., 2008)and human diseases, including cancer, Parkinson's disease, Crohn's disease, liposome storage disorders, and so forth (van Beek et al., 2018).

\section{The process of autophagy}

Autophagy is a tightly regulated multi-stage activity, which can be dissected into 6 steps: initiation, phagophore nucleation, phagophore membrane expansion and cargo engulfment, autophagosome fusion with the vacuole or lysosome, cargo degradation, and recycling of the processed materials (Mizushima, 2007). Triggered by a wide range of signaling pathways, autophagy is initiated by activation of the unc-51like kinase (ULK) complex. This step sequentially activates the phosphoinositide 3kinase (PI3K) complex and Atg9 which collectively mediate the phagophore nucleation (Lane et al., 2017). Once the nucleation is completed, membrane from multiple potential sources, for example mitochondria (Lyamzaev et al., 2018; Rambold and Lippincott-Schwartz, 2011), endosomes (Longatti et al., 2012; Puri et al., 2013), endoplasmic reticulum (ER) (Carlos Martín Zoppino et al., 2010), Golgi bodies (Bodemann et al., 2011; van der Vaart et al., 2010), and even plasma membrane (Ravikumar et al., 2010), gathers at the phagophore as forming a double membrane organelle called autophagosome (Carlsson and Simonsen, 2015). This process is followed by other autophagic machinery being recruited to the isolated membrane preparing for the membrane elongation. The key proteins include WD-repeat protein interacting with phospho-inositides (WIPI) proteins (Proikas-Cezanne et al., 2015) and the enzyme cascade required for Atg 8 family protein lipidation (Rubinsztein et al., 2012). Atg8 family is a distinct subgroup of the ubiquitin-like (UBL) proteins. They modify phagophore membrane lipids through a chemical reaction similarly to other 


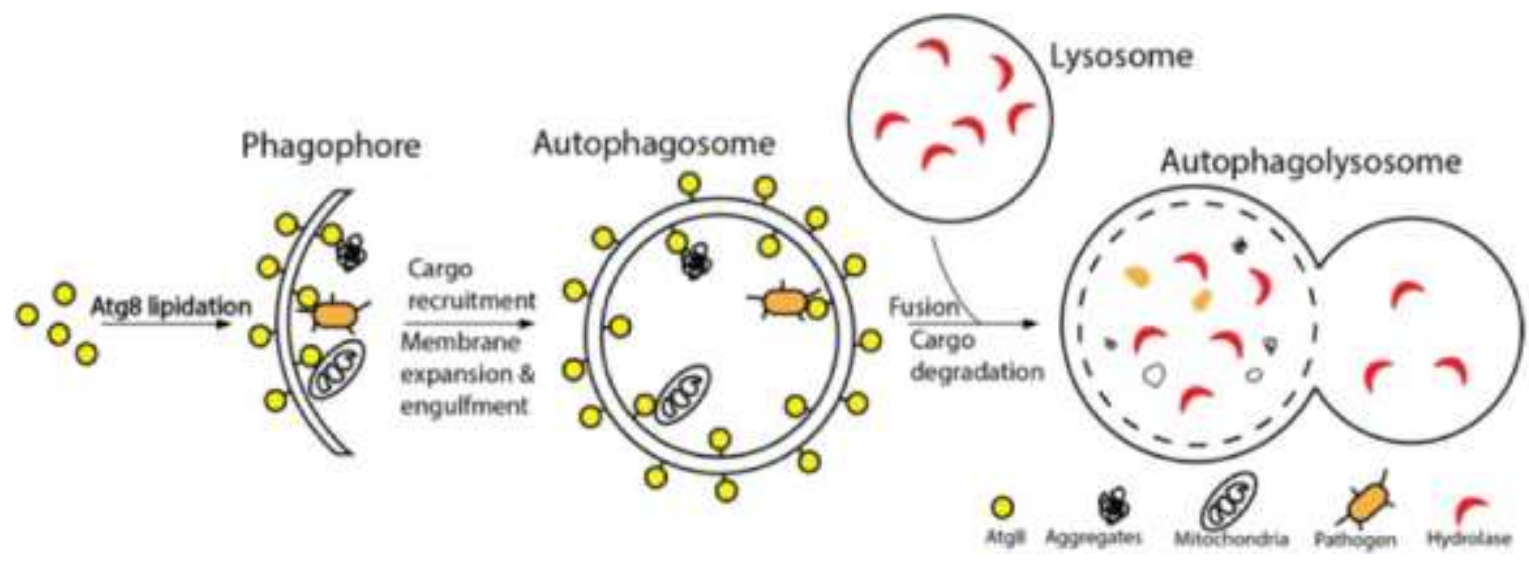

Figure 1-1. The process of autophagy.

After Atg8/ LC3 family proteins being ligation to the phosphatidylethanolamine(PE) on phagophore membrane is followed by membrane elongation and engulfment, meanwhile autophagic cargos are recruited to the phagophore via Atg8 binding with a variety of specific adaptor proteins that recognize and interact with the cargos, forming a the double membrane vesicle termed as autophagoosome. Autophagosome fuses with lysosome (or vacuole in yeast) and utilizes the lysosomal hydrolases to degrade the cargos for reutilization. 
ubiquitin-like protein ligations, featured by the glycine at the truncated C-terminus of Atg 8 family proteins being covalently ligated to amino group of phosphatidylethanolamine (PE) (Nakatogawa et al., 2007). Membrane-linked Atg8 family proteins not only participate in the phagophore elongation and fusion, but also promote autophagosome closure (Feng et al., 2014; Fujita et al., 2008a). Moreover, autophagosome-coated Atg8 family proteins are responsible for autophagic receptor recognition and recruitment. This activity is realized by a specific interaction, where a hydrophobic groove on Atg8 captures unique peptide sequences termed as the Atg8 interactive motif (AIM, in yeast or fungi) or LC3 interactive region (LIR, in higher organisms) harbored by autophagic adaptors or other autophagy related binding partners (Birgisdottir et al., 2013). As phagophore membrane expands and cargo engulfment proceeds, autophagosome eventually seals, then most of Atg8 molecules are cleaved from the outer-membrane by Atg4 . In the next step, autophagosome first migrates with facilitation from the microtubule system, then fuse with vacuole or lysosome under the regulation of soluble $\mathrm{N}$-ethylmaleimide-sensitive factor attachment protein receptor (SNARE), Rab family proteins, PI3K complex and Rubicon (Hikita et al., 2018). The inner membrane and the engulfed cargos of the autophagsome are therefore degraded by vacuolar or lysosomal hydrolases. Finally, the resulting metabolites are exported to the cytosol for reutilization.

\section{Atg8 family protein lipidation and autophagy}

Autophagy-related 8 protein (Atg8) family is conserved across eukaryotic species. Atg8 is the only family member found in yeast and other fungus, whereas other three subfamilies: microtubule-associated protein 1 light chain 3 (MAP1LC3 or LC3), $\gamma$ aminobutyric acid receptor-associated protein (GABARAP) and Golgi-associated ATPase enhancer of $16 \mathrm{kDa}$ (GATE-16) exist in higher organisms including animals and plants. Despite residue variation in several positions across the whole proteins, Atg8 family members share major similarity in amino acid sequences and protein structures (Figure 1-2). Structural studies in different Atg8 family proteins, including, but not limited to, Atg8s from multiple yeast organisms and the human LC3 and GABARAP family members, have revealed significant conformational similarity to ubiquitin. However, comparing to other UBL family members, in addition to the globular ubiquitin domain, Atg8 family proteins feature an additional N-terminal tail with moderate flexibility which consists of two helices connected by a short linker (Figure 1-2).

As described in 1.1.2, many facets of autophagy rely on Atg8 in yeast (or six LC3- and GABARAP-family ubiquitin-like proteins in higher eukaryotes, here referred collectively to Atg8 family members) and the E1-E2-E3 enzymatic cascade that links Atg8's C-terminus to the primary amine group of phosphatidylethanolamine (PE) lipid molecules (Dikic, 2017; Ichimura et al., 2000; Shpilka et al., 2011, 2012). As initiation of the lipidation process, the C-terminal tail of Atg8 family protein is cleaved by Atg4, exposing a glycine residue at the very end accessible by nucleophiles. Under tight regulation of autophagic machinery, this truncated version of Atg8 forms a thioester bond-linked complex with Atg7 (E1) via sidechains of C-terminal glycine and Atg7 


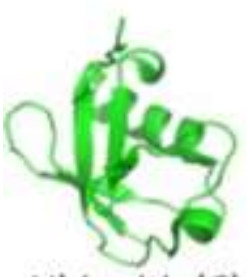

Ubiquitin(C)

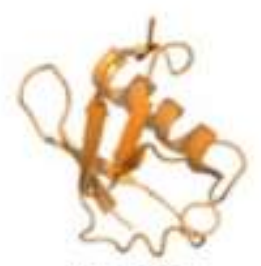

NEDD8

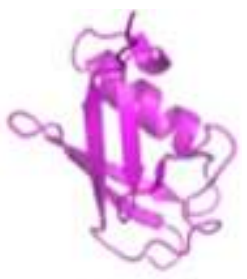

SUMO

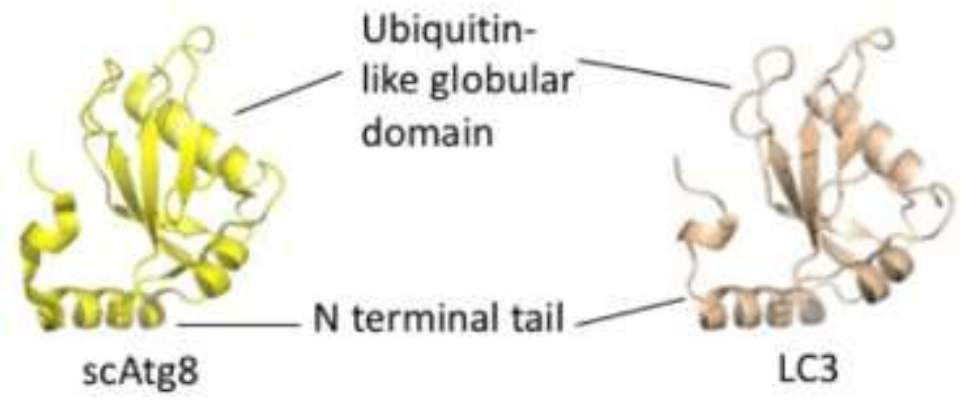

Figure 1-2. Structure comparison of Atg8 and LC3 to other ubiquitin like proteins.

In addition to the globular domain that shared by all ubiquitin-like proteins, Atg8/LC3 family proteins are featured by a N-terminal helical extension. 
catalytic cysteine. Ultimately, lipidated Atg8 family members serve as bridges between membranes and the cytosol, where their ubiquitin-like fold domains recruit AIM or LIR sequences in a staggering array of partner proteins involved in various aspects of autophagy (Birgisdottir et al., 2013; Noda et al., 2010; Rogov et al., 2014; Wild et al., 2014). These include regulators and effectors of autophagosome biogenesis that promote membrane tethering and growth of the autophagosome, as well as adaptor proteins that in turn recruit cargoes for degradation (Zaffagnini and Martens, 2016). Accordingly, Atg8 and its conjugation system enzymes are essential for autophagy in yeasts, and autophagy is severely impaired in their absence in mammalian cells (Tsuboyama et al., 2016). Lipidated Atg8-family members have also been implicated in protein recruitment to membranes in processes that are unrelated to autophagy in mammalian cells. Therefore, given these important roles of Atg8 and its conjugation system enzymes, it is important to understand molecular mechanisms regulating Atg8 lipidation.

\section{The process of Atg8 lipidation}

The process of Atg8 lipidation largely follows the similar procedure of other ubiquitin-like protein ligation system, whereas the ubiquitin or ubiquitin-like protein is delivered through a three enzyme (E1, E2, and E3) cascade before being conjugated to the final substrate (Ohsumi and Mizushima, 2004). The UBL, or Atg8 processed by Atg4 in this context, first uses its $\mathrm{C}$-terminal glycine to generate a thioester bond with the catalytic cysteine in E1 Atg7 (Tanida et al., 1999); this E1-UBL complex is disassociated upon interacting with E2 Atg3, where UBL is transferred to E2 and forms a new thioester bond with the catalytic cysteine of E2_(Tanida et_al..22002); finally, facilitated by E3 Atg12-Atg5-Atg16, UBL is discharged from E2 and ligated to the target amine group located within the substrate PE (Hanada et al., 2007; Walczak and Martens, 2013) (Figure 1-3). While many UBL family members are able to coordinate with diverse E2s and E3s, and target an even wider range of substrates, Atg8, however, stays strictly specific to E2-like protein Atg3, E3-like protein complex Atg12-Atg5-Atg16 and it's only lipid substrate - phosphatidylethanolamine (PE); Atg8 is also known as the only UBL that modifies amine group in lipid substrates other than lysines in protein substrates.

Atg7, the E1 for Atg8, harbors a N-terminal domain (NTD) and a C-terminal domain (CTD) (Taherbhoy et al., 2011). Atg7 adenylation domain (AD), which is a subdomain of the CTD, mediates self-dimerization as ADs in the canonical E1, and is both structurally and functionally comparable to the canonical E1 AD dimers (Taherbhoy et al., 2011). The flat dimeric interface serves as a platform to interact with the UBL as in other E1s. However, lacking the E1's signature ubiquitin-fold domain (UFD) but replacing it by the unique NTD, the overall structure of Atg7 is distinct from other E1s. Unlike canonical E1 using UFD to recruit E2, Atg7, as dimerized via AD, interacts with Atg3 core domain by one copy of NTD in cis, and meanwhile binds an exclusive motif in Atg3 that is absent in other canonical E2s by the other copy in trans (Taherbhoy et al., 2011). 


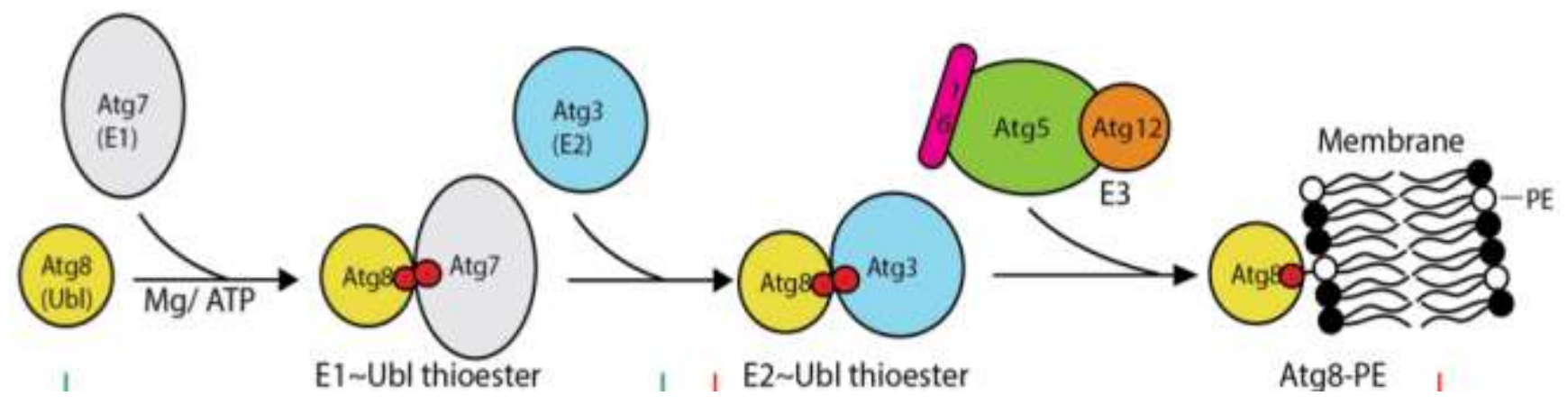

Figure 1-3. The process of Atg8 lipidation.

The lipidation of Atg8/LC3 family protein utilizes E1-E2-E3 cascade similar to other ubiquitin-like protein conjugation system. Atg8 first forms a thioester intermediate with activated E1 Atg7; he thioester bond is nucleophilic attacked by Atg3 catalytic cysteine, resulting in breaking down of the Atg7-Atg8 bond and formation a new thioester bond between Atg8 with E2 Atg3 catalytic cysteine; in the final step, E3 Atg12-Atg5-Atg16 facilitates Atg8 being discharged from Atg3 and ligated to PE. 
The unique E2 for Atg8, Atg3, features a core domain that resembles other canonical E2 folds and harbors an E2 catalytic center including a topologically conserved catalytic cysteine (Yamada et al., 2007). In addition, it contains a 20-amino acid Nterminal helical extension (N-term) with the ability of sensing membrane curvature (Nath et al., 2014), an approximately 100-amino acid unstructured insertion in the middle of the core domain termed as the flexible region (FR) (Yamada et al., 2007), and an unique handle region (HR) downstream of the catalytic cysteine (Yamada et al., 2007). Upon activation by the E3-like enzyme Atg12-Atg5-Atg16 complex, the Atg3 catalytic center undergoes conformational changes, during which the catalytic cysteine and its surrounding regulative residues are rearranged to positions favored by Atg8-ligation (Sakoh-Nakatogawa et al., 2013). However, the functions of the FR and HR in Atg8 lipidation remain obscure, though human protein study suggested that a short motif within the FR adopts a helical folding upon binding to the E3 compartment ATG12 (Metlagel et al., 2013). And such interaction is indispensable for LC3 conjugation activity. Due to lack of structural evidences, it is also unrevealed about the full conformational requirements to activate the Atg3 Atg8 thioester intermediate as well as the full picture of Atg3-Atg12-Atg5-Atg16 interaction.

The E3-like enzyme Atg12-Atg5-Atg16 resembles the ubiquitin-like protein ligation process. As a ubiquitin-like protein itself, Atg12 is conjugated to the substrate Atg5 via an isopepetide bond, catalyzed by its E1 Atg7 and E2 Atg10 without involvement of an E3 (Hanada and Ohsumi, 2005; Ohsumi and Mizushima, 2004). Atg16 later joins the Atg12-Atg5 complex as a non-covalent yet stable binding partner. Both human ATG5 and ATG16L were revealed to have intrinsic binding abilities to lipids (Lystad et al., 2019; Romanov et al., 2012). Taken together with the membrane curvature sensing function of the ATG3 N-terminal helix (Nath et al., 2014), this may indicates a potential recruiting mechanism of the Atg3 Atg8 intermediate to the phagophore membrane. Neither the Atg12-Atg5-Atg16 E3 complex nor its individual compartments shows conservation in sequence to any of the three families of E3 (the RING family, the HECT family, and the RBR family); studies in multiple organisms including human and yeast also reveal no structural similarity between Atg12-Atg5-Atg16 and other E3 ligases. With these significant differences between autophagy and canonical E1, E2, and E3s, it is challenging to study the mechanism of Atg8 lipidation based on existing UBL conjugation models. On the other hand, all these distinctive features of the Atg8 lipidation cascade give more significance to study Atg8 lipidation, not only to complete the puzzle of autophagic pathway, but also to expand the current understanding of ubiquitin-like protein modification machineries.

\section{Atg8 family protein lipidation cascade and diseases}

As a unique protein family serving as an interactive hub to recruit other proteins to the autophagic membrane, lipidated Atg8 family members are critical in many essential biological pathways. Previous studies have implemented mouse models deficient in core autophagy genes to investigate physiological and pathogenic effects. 
Previous studies in human patients showed that $A T G 7$ polymorphisms (SNPs) at $100 \mathrm{~A}>\mathrm{G}$ and $25108 \mathrm{G}>\mathrm{A}$ promote the severity of asthma, likely by inducing higher level of interleukin-8 (IL-8) secretion to the serum, which potentially causes systematic inflammation (Shao et al., 2017). Moreover, changes in ATG7 promotor region $(11313449 \mathrm{G}>\mathrm{A}, 11313811 \mathrm{~T}>\mathrm{C}, 11313913 \mathrm{G}>\mathrm{A}$ and $11314041 \mathrm{G}>\mathrm{A})$ result in excessive expression of ATG7 protein, which correlates with aggravated brain damage in Parkinson disease patients (Chen et al., 2013). In addition, substitution of human ATG7 Val471 with alanine has shown association with an average 4-year earlier Huntington disease (HD) onset in European patients (Metzger et al., 2010).

Beside the E1 ATG7, all three compartments of the E3 Atg12-Atg5-Atg16 harbor significant functions in a wide collection of diseases including asthma, lupus, autoimmune disorder, bone disease, neuron degeneration, and cancer. The SNP localized in ATG5 putative promotor region (rs12201458) has been shown negative impacts on asthma prognosis, probably due to an increase in the total ATG5 level in nasal mucosal cells, which has been observed in both acute and long-term asthma patients (Martin et al., 2012). Two of the ATG5 SNPs (rs6937876 and rs548234) were also linked to multiple risk factors in systemic lupus erythematosus (SLE) disease onset (Qi et al., 2018; Zhou et al., 2011). Regarding to Behcet disease (BD), one of the ATG5 genetic changes (rs573775) was proposed to have protective function by a study in a Chinese patient cohort (Zheng et al., 2015). Another SNP in ATG5 (rs2245214) is associated with enhanced chance of developing Paget disease of bone (PDB) (Usategui-Martín et al., 2015). Even a subtle mutation E122D in ATG5 that leads to moderate structural changes causes ataxia (Kim et al., 2016). Moreover, studies in clinical patients also linked several ATG5 SNPs with higher hepatocellular carcinoma (HCC) risk (Shen and Lin, 2019). Atg16 also plays an indispensable role in numerous disease onset or progression. For example, different SNPs occurring at the same site of human ATG16L1 (rs2241880, T300), can confer various outputs in patient studies (Chen et al., 2015; Li et al., 2017a; Usategui-Martín et al., 2015). Change of the local deoxynucleotide to A, with an unchanged coding for T300, is related to increased risk of chronic obstructive pulmonary (COPD) (Chen et al., 2015). ATG16L1 C allele mutation at this site was shown associated with Paget disease of bone (Li et al., 2017a). Additionally, the G SNP coding for T300A was linked to the likelihood of developing Crohn disease (Chen et al., 2015). However, in another study, the T300A variant of ATG16L1 was associated with decreased risk of brain metastasis of in non-small cell lung cancer patients (Li et al., 2017a). Another ATG16L1 SNP rs4663402 has strong relation with greater hepatocellular carcinoma risk (Shen and Lin, 2019). Nonetheless, polymorphisms in ATG12 promotor region (Li et al., 2017b) and protein coding region (Shen and Lin, 2019) result in enhanced occurrences of Parkinson disease and hepatocellular carcinoma in human respectively.

\section{Summary and Aims}

Autophagy is a fate-determining metabolic activity to recycle cellular material and to avoid cell death under unfavored external and internal conditions. During the 
course of autophagy, ligation of Atg8 family protein to PE lipid on both side of the autophagsome membrane (Atg8 lipidation) serves as an indispensable and tightly regulated check point, which plays significant roles in phagophore nucleation, membrane extension, autophagosome engulfment, vacuole/lysosome fusing with autophagosome, and cargo recruiting. As such, in order to decipher autophagy in a comprehensive and detailed level, it is important for us to understand the molecular mechanism of Atg8 family protein ligation to the membrane.

In this study, we will introduce a variety of methods, including but not limited to biochemistry, structural biology and cell biology approaches, to investigate the molecular requirements for Atg8/LC3 lipidation in human as well as Saccharomyces Cerevisiae system. The results will potentially shed light on therapy development and drug design that target the Atg8 lipidation cascade serving for modulation of the autophagy pathway. 


\section{CHAPTER 2. PRODUCTION OF HUMAN ATG PROTEINS FOR LIPIDATION ASSAYS ${ }^{1}$}

\section{Introduction}

In eukaryotic cells, autophagy is a tightly regulated process responsible for degrading and recycling cellular components, and eliminating toxic assemblies and pathogens by sequestering cargo within an autophagosome, which fuses with a lysosome (or vacuole in yeast) harboring hydrolytic enzymes (Klionsky, 2000; Wileman, 2013). Several key aspects of this regulation involve ubiquitin-like proteins (UBLs) in the Atg8 family, which form LC3 and GABARAP subfamilies in higher eukaryotes.

LC3/GABARAP proteins have molecular weights in solution of approximately 14-17 $\mathrm{kDa}$, but their electrophoretic migration is relatively accelerated upon ligation to lipids such as in autophagic membranes. LC3/GABARAP ligation to lipids (lipidation) plays numerous roles in regulation, including influencing the formation, maturation, and size of autophagosomes, as well as recruiting selected cargoes and regulatory proteins (Ichimura et al., 2000; Nakatogawa et al., 2007; Ohsumi and Mizushima, 2004; Tanida et al., 2008)

LC3 and GABARAP lipidation is orchestrated by a UBL ligation system, specifically conjugating the carboxyl group of the C-terminal glycine of LC3/GABARAP to the amine group of phosphatidylethanolamine (PE) (or potentially other lipids such as phosphatidyl serine) in autophagic membranes (Hanada et al., 2007; Ichimura et al., 2000; Klionsky, 2000; Sou et al., 2006; Tanida et al., 2004b, 2004c). This involves a specialized enzyme cascade, where ATG7 acts as the E1 and ATG3 acting as the E2 to produce covalent, reactive thioester-linked ATG3 LC3/GABARAP (“ " denotes thioester bond and "-" denotes a noncovalent complex) intermediates. Efficient lipidation requires an ATG12-ATG5-ATG16L1 multiprotein complex acting as the E3. Notably, formation of this E3-like assembly first requires a related conjugation cascade, wherein the UBL ATG12 is covalently conjugated to ATG5 by ATG7 acting as the E1 and ATG10 acting as the E2 (Hanada et al., 2007; Noda et al., 2013), and then the ATG12ATG5 binds ATG16L1 to achieve full E3-like activity toward ATG3 LC3/GABARAP intermediates. Mutations or dysfunction of proteins in the LC3/GABARAP lipidation cascade results in severe diseases and poor prognosis in various eukaryotic organisms including humans (Cadwell et al., 2009; Kim et al., 2016; Lenz et al., 2011; Moloughney et al., 2011; Reed et al., 2015; Tindwa et al., 2015; Wu et al., 2014; Xue et al., 2010).

Given the importance of LC3 and GABARAP lipidation, and potential interest in targeting their conjugation for therapeutic purposes (Cheng et al., 2013; Puri and Chandra, 2014), purified proteins for assaying LC3/GABARAP lipidation and performing structural analyses would be useful (Kaiser et al., 2013; Metlagel et al., 2013, 2014; Nath et al., 2014; Otomo et al., 2013; Sakoh-Nakatogawa et al., 2013; Taherbhoy

\footnotetext{
${ }^{1}$ Reprinted from final submission with permission. Zheng, Y., Qiu, Y., Gunderson, J.E.C., and Schulman, B.A. (2017). Production of Human ATG Proteins for Lipidation Assays. In Methods in Enzymology, (Elsevier), pp. 97-113. doi: 10.1016/bs.mie.2016.09.055
} 
et al., 2011). However, our optimal expression and purification procedures differ between the human LC3/GABARAP lipidation components and their yeast counterparts (Qiu et al., 2013). Thus, in this chapter, we describe detailed methods for expression and purification of human ATG proteins involved in LC3/GABARAP lipidation, including ATG7, ATG3, ATG12-ATG5-ATG16L1, and a method for expressing LC3B as exemplary for the human Atg8 orthologues.

\section{Expression of Human LC3B, ATG7, ATG3, ATG12-ATG5, and ATG16L1}

\section{General overview of protein expression and purification}

LC3B (emblematic of LC3/GABARAP family members), ATG3, and ATG16L1 were expressed as GST-fusion proteins in Escherichia coli, whereas ATG7 and the ATG12-ATG5 conjugate were expressed through distinct methods in insect cells (Berger et al., 2004). An overview of the subsequent purification strategies is shown in Figure 2-1.

\section{Expression constructs}

A truncated version of LC3B, corresponding to residues 1-120 that form the activated form with a $\mathrm{C}$-terminal glycine that is the site of chemical reactions (Tanida et al., 2004b, 2004c), is expressed from pGEX-4T-1 (Table 2-1, Figure 2-2a) in E. coli. Full-length ATG7 was expressed from a modified version of pFastBac, with TEVcleavable N-terminal GST tag (pFastBac-GST) (Table 2-1, Figure 2-2b) for expression in insect cells. Full-length ATG3 was expressed from a modified version of pGEX-4T-1 harboring a cleavage site for TEV protease instead of thrombin between GST and ATG3 (pGEX-TEV) (Table 2-1, Figure 2-2c), for expression in E. coli. A truncated version of ATG16L1 corresponding to residues 1-69 was expressed from pGEX-TEV (Table 2-1, Figure 2-2d) in E. coli.

The ATG12-ATG5 subcomplex corresponds to a truncated version of ATG12 (residues 52-140) that comprises the ubiquitin-like domain (Hanada and Ohsumi, 2005) linked via an isopeptide bond between its C-terminus to Lys130 on full-length ATG5 (Hanada et al., 2007; Otomo et al., 2013). The linkage between ATG12 and ATG5 is achieved by their coexpression with ATG7 and ATG10 in insect cells using the biGBac system (Weissmann et al., 2016). First, four separate plasmids were generated. The coding sequences were inserted into pLIB vectors, with ATG7, and ATG10 untagged, ATG5 harboring a N-terminal TEV cleavable His-tag, and ATG12 (52-140) with a Nterminal TEV cleavable GST-tag (Table 2-1, Figure 2-2e). Next, GST-TEV-ATG12 (52-140), His-TEV-ATG5, ATG10, and ATG7 along with the promoter and terminator from the pLIB vectors were sequentially amplified using primers $\mathrm{d}$ scribed in Weissmann et al., 2016, and coassembled into the pBIG1a vector (Table 2-1, Figure 2-2f) using the 


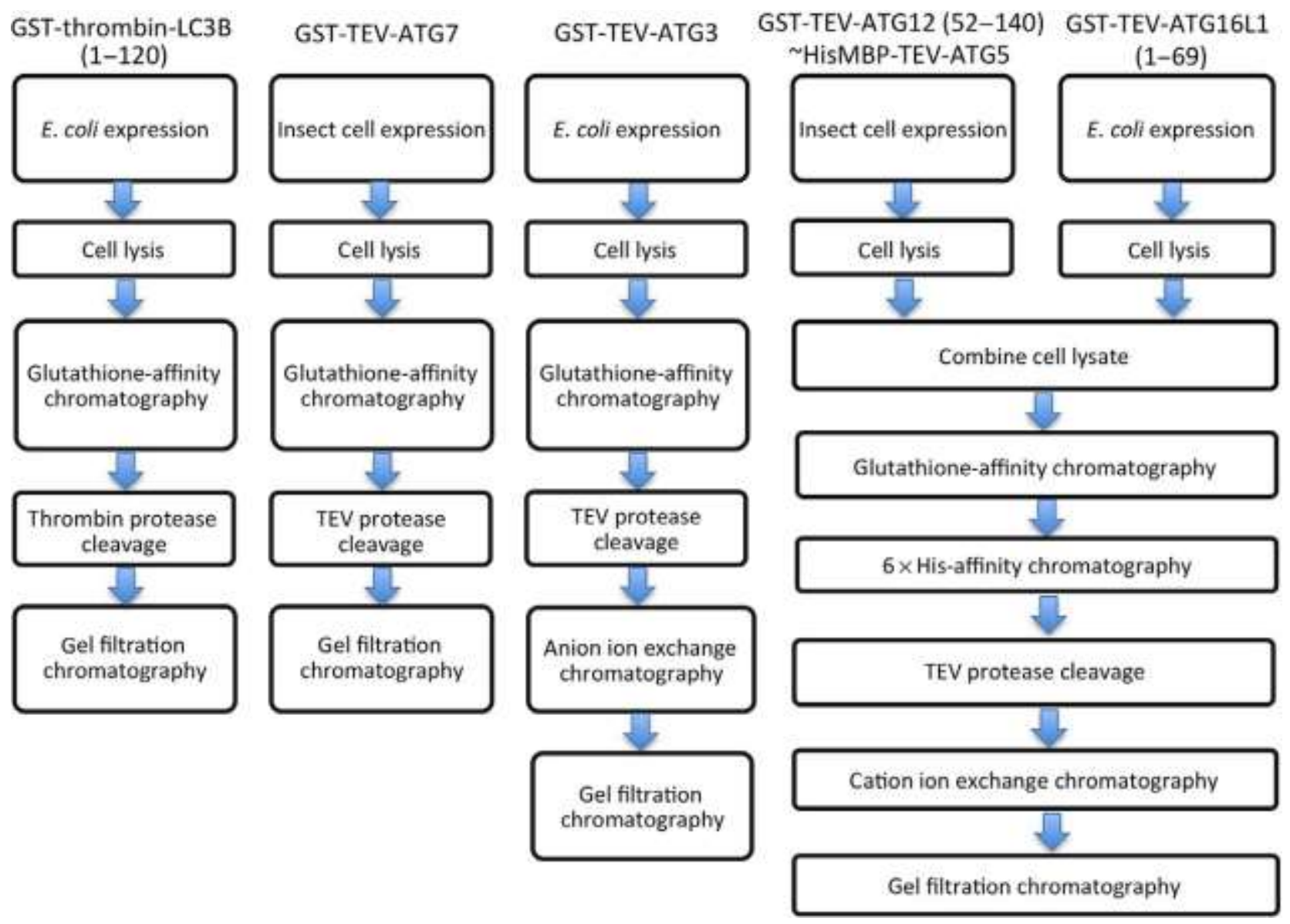

Figure 2-1. Flow-chart indicating the expression system and purification strategies for production of human ATG proteins for lipidation assays. 
Table 2-1. Plasmids used to produce human ATG proteins for lipidation assays.

\begin{tabular}{|c|c|c|}
\hline Protein expressed & Plasmids generated & Functions \\
\hline LC3B (1-120) & pGEX-4T-1-LC3B & Protein expression in $E$. coli \\
\hline ATG7 & pFastBac-GST-ATG7 & $\begin{array}{l}\text { To generate bacmid for protein } \\
\text { expression in insect cells }\end{array}$ \\
\hline ATG3 & pGEX-TEV-ATG3 & Protein expression in $E$. coli \\
\hline ATG12 (52- & pLIB-GST-TEV-ATG12 & To generate $\mathrm{pBIG} 1 \mathrm{a}$ plasmid \\
\hline \multirow[t]{4}{*}{ 140) ATG5 } & pLIB-His-TEV-ATG5 & To generate $\mathrm{pBIG}$ la plasmid \\
\hline & pLIB-ATG10 & To generate pBIG1a plasmid \\
\hline & pLIB-ATG7 & To generate $\mathrm{pBIG}$ la plasmid \\
\hline & $\begin{array}{l}\text { pBIG1a-ATG12-ATG5- } \\
\text { ATG10-ATG7 }\end{array}$ & $\begin{array}{l}\text { To generate bacmid for protein } \\
\text { expression in insect cells }\end{array}$ \\
\hline ATG16L1 (1-69) & pGEX-TEV-ATG16L1 & Protein expression in E. coli \\
\hline
\end{tabular}



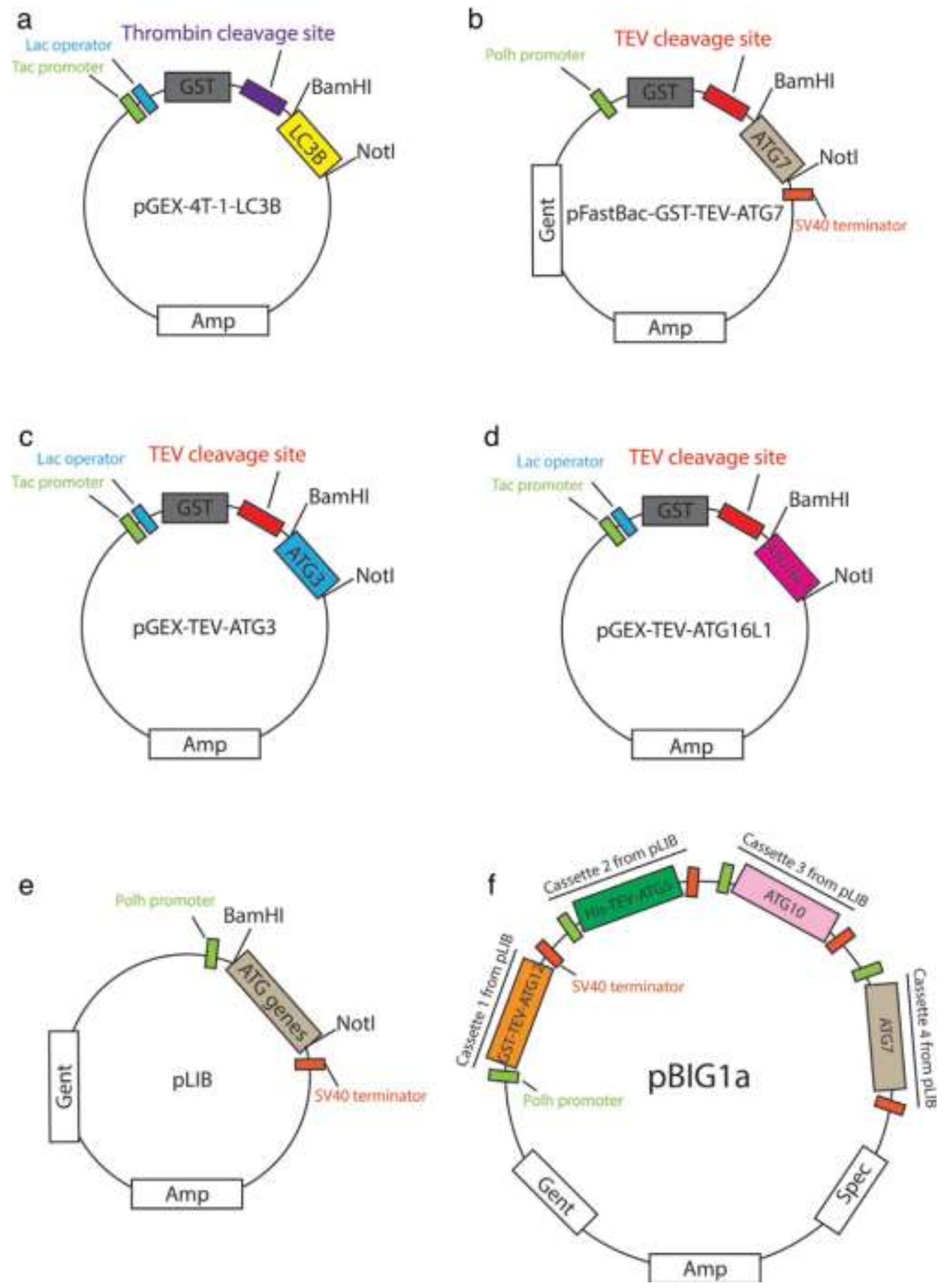

Figure 2-2. Graphical representation of plasmids used in this study.

a. pGEX-4T-1-LC3B for LC3B (1-120) expression in E. coli. b. pFastBac-GST-ATG7 to construct bacmid for ATG7 expression in insect cells. c. pGEX-TEV-ATG3 for ATG3 expression in E. coli. d. pGEXTEV- ATG16L1 for ATG16L1 (1-69) expression in E. coli. e. pLIB plasmid containing a GSTTEV-ATG12 (52-140), His-TEV-ATG5, ATG10, or ATG7 expression cassette to generate pBIG1a plasmid. f. pBIG1a-ATG12-ATG5ATG10-ATG7 plasmid to construct bacmid for ATG12 (52-140)-ATG5 expression in insect cells. 
method of Gibson Assembly (Gibson, 2011; Gibson et al., 2009; Weissmann et al., 2016).

All constructs were generated through transformation into DH5alpha (NEB), bacterial growth, minipreps, and verified by automated sequencing. The recommended antibiotic concentrations are: $100 \mu \mathrm{g} / \mathrm{mL}$ for ampicillin, $50 \mu \mathrm{g} / \mathrm{mL}$ for kanamycin and spectinomycin, $25 \mu \mathrm{g} / \mathrm{mL}$ for chloramphenicol, $10 \mu \mathrm{g} / \mathrm{mL}$ for tetracyclin, and $7 \mu \mathrm{g} / \mathrm{mL}$ for gentamycin.

\section{Protein expression}

\section{Expression in E. coli}

Plasmid was transformed into BL21-CodonPlus (DE3)-RIL E. coli competent cells (Agilent Technologies). Bacteria was plated on LB-agar with appropriate antibiotics and Incubated at $37^{\circ} \mathrm{C}$ for $12-18 \mathrm{~h}$. The starter culture was prepared from a single colony in $5 \mathrm{~mL} \mathrm{LB}$ medium with antibiotics, and was cultured at $37^{\circ} \mathrm{C}, 200 \mathrm{rpm}$ for $10-16 \mathrm{~h}$. Then $5 \mathrm{~mL}$ of starter culture was inoculated into $1 \mathrm{~L} \mathrm{LB}$ medium with appropriate antibiotics. The cells were grow at $37^{\circ} \mathrm{C}, 200 \mathrm{rpm}$, and monitor closely by measuring the absorbance at $600 \mathrm{~nm}$. When OD600 reaches 0.8-1.0, IPTG was added at a final concentration of $0.6 \mathrm{mM}$. Shaker temperature was reset to $18^{\circ} \mathrm{C}$, allowing growing with shaking at $200 \mathrm{rpm}$ for $16-20 \mathrm{~h}$.

\section{Expression in insect cells}

For GST-thrombin-LC3B, GST-TEV-ATG7, and GST-TEV-ATG3, glutathioneaffinity chromatography was directly applied to their corresponding cell lysates. To obtain GST-TEV-ATG12 (52-140)-His-TEV-ATG5-GST-TEV-ATG16L1 (1-69) complex, cell lysates from insect cells and bacteria were mixed at 1:1 volume ratio and incubated at $4^{\circ} \mathrm{C}$ for $10 \mathrm{~min}$ before processing.

I washed Glutathione Sepharose 4B (GS4B, GE Healthcare Life Sciences) 3 times with 10 column volumes of wash buffer in a chromatography column at $4{ }^{\circ} \mathrm{C}$. Alternatively, the beads could also be washed with 3 volumes of wash buffer by centrifugation at $500 \mathrm{x}$ g for $5 \mathrm{~min}$ at $4^{\circ} \mathrm{C}$, decanting the wash buffer, and repeating twice. Suggested bead volumes: $0.75 \mathrm{~mL}$ per $1 \mathrm{~L}$ bacterial culture, or $2 \mathrm{~mL}$ per $1 \mathrm{~L} \mathrm{Hi} 5$ insect cell culture, which is appropriate for both GST and nickel beads and all species of ATG proteins described in this chapter. Wash buffer was the same as lysis buffer but without PMSF, aprotinin, leupeptin, or any other proteasome inhibitor.

Lysate was incubated with washed beads. The mixture was gently rocked at $4^{\circ} \mathrm{C}$ for $2 \mathrm{~h}$ for GS4B beads or $1 \mathrm{~h}$ for nickel beads to allow full binding.

Then lysate with beads were transferred into a Poly-Prep chromatography column (Bio-Rad) at $4^{\circ} \mathrm{C}$. Lysate was allowed to flow through by gravity to generate an even 
resin surface in column. Although the flow-through is unneeded in principle, it is a good practice to save the flow-through for troubleshooting in the event of problems.

If the lysate-bead solution is too thick to pass through the column, I would centrifugate the solution in $50 \mathrm{~mL}$ tubes at $500 \mathrm{x}$ g for $5 \mathrm{~min}$ at $4{ }^{\circ} \mathrm{C}$. The supernatant was collected as flow through. Pelleted beads was gently resuspended in 10 column volumes of wash buffer and washed following last step.

Finally, resin was washed in column with 10 column volumes of wash buffer without disturbing the flat resin surface. This step was repeated 3 times.

\section{Protein Purification}

\section{Preparation of cell lysate}

Bacteria cells were harvested by centrifugation at $5900 \mathrm{x}$ g for $12 \mathrm{~min}$ at $4^{\circ} \mathrm{C}$. Insect cells were harvested at $500 \mathrm{x}$ g for $15 \mathrm{~min}$ at $4^{\circ} \mathrm{C}$.

Cell pellet was suspended in $15 \mathrm{~mL}$ lysis buffer per liter bacteria culture or $50 \mathrm{~mL}$ per liter insect cell culture. Lysis buffer for all proteins except the ATG12-ATG5 subcomplex was: $25 \mathrm{mM}$ Tris- $\mathrm{HCl} \mathrm{pH}$ 7.6, $200 \mathrm{mM} \mathrm{NaCl}, 2.5 \mathrm{mM}$ phenylmethanesulfonyl fluoride (PMSF), $5 \mathrm{mM}$ 2-Mercaptoethanol (BME). Lysis buffer for ATG12-ATG5 complex was: 1 x PBS, $400 \mathrm{mM} \mathrm{NaCl,} 2.5 \mathrm{mM}$ PMSF, $5 \mathrm{mM}$ BME. Also add $20 \mathrm{mg} / \mathrm{L}$ aprotinin, $10 \mathrm{mg} / \mathrm{L}$ leupeptin, or a protease inhibitor cocktail of choice to insect cell lysis buffer.

Bacteria cells was lysed by sonicating 7 times, or insect cells 3 times, $10 \mathrm{~s}$ each time with intermittent cooling on ice.

Lysate was cleared by centrifugation at $32,000 \mathrm{x}$ g for $1 \mathrm{~h}$ at $4{ }^{\circ} \mathrm{C}$. The supernatant was collected and kept on ice.

\section{Glutathione-affinity chromatography for LC3B, ATG7, ATG3, and ATG12-ATG5- ATG16L1}

For GST-thrombin-LC3B, GST-TEV-ATG7, and GST-TEV-ATG3, glutathioneaffinity chromatography was directly applied to their corresponding cell lysates. To obtain GST-TEV-ATG12 (52-140)-His-TEV-ATG5-GST-TEV-ATG16L1 (1-69) complex, cell lysates from insect cells and bacteria were mixed at 1:1 volume ratio and incubated at $4^{\circ} \mathrm{C}$ for $10 \mathrm{~min}$ before processing.

Glutathione Sepharose 4B (GS4B, GE Healthcare Life Sciences) was washed 3 times with 10 column volumes of wash buffer in a chromatography column at $4{ }^{\circ} \mathrm{C}$. 
Alternatively, the beads could also be washed with 3 volumes of wash buffer by centrifugation at $500 \mathrm{xg}$ for $5 \mathrm{~min}$ at $4^{\circ} \mathrm{C}$, decanting the wash buffer, and repeating twice. Bead volumes: $0.75 \mathrm{~mL}$ per $1 \mathrm{~L}$ bacterial culture, or $2 \mathrm{~mL}$ per $1 \mathrm{~L}$ Hi5 insect cell culture, which was appropriate for both GST and nickel beads and all species of ATG proteins described in this chapter. Wash buffer was the same as lysis buffer but without PMSF, aprotinin, leupeptin, or any other proteasome inhibitor.

The lysate was mixed with washed beads. The mixture was gently rocked at $4{ }^{\circ} \mathrm{C}$ for $2 \mathrm{~h}$ for GS4B beads or $1 \mathrm{~h}$ for nickel beads to allow full binding.

Lysate with beads were transferred into a Poly-Prep chromatography column (Bio-Rad) at $4^{\circ} \mathrm{C}$. Lysate were allowed to flow through by gravity to generate an even resin surface in column. Although the flow-through is unneeded in principle, it is a good practice to save the flow-through for troubleshooting in the event of problems.

If the lysate-bead solution is too thick to pass through the column, I would centrifugate the solution in $50 \mathrm{~mL}$ tubes at $500 \mathrm{xg}$ for $5 \mathrm{~min}$ at $4^{\circ} \mathrm{C}$. The supernatant was collected as flow through. Pelleted beads was gently resuspended in 10 column volumes of wash buffer and follow last step.

The resin was washed in column with 10 column volumes of wash buffer without disturbing the flat resin surface. This step was repeated 3 times.

\section{Protease-mediated elution from beads for purification of LC3B, ATG3, and ATG7}

Rough protein concentration of bead turbid solution was measured using Bio-Rad protein assay with a standard curve. Bovine serum albumin is a suitable standard. $10 \mu \mathrm{L}$ of uncleaved sample was stored as a negative control for cleavage efficiency check before adding protease.

Thrombin protease was added to LC3B (1 $\mathrm{mg}$ thrombin per $100 \mathrm{mg}$ of total protein), or TEV protease to ATG7 and ATG3 (2 $\mathrm{mg}$ TEV per $100 \mathrm{mg}$ total protein). The mixture was kept gently rotated on a rocking shaker overnight to allow full cleavage. To be certain that all desired protein is cleaved from the GST-tag, I examined the bead slurry by SDS-PAGE, using a 15\% acrylamide gel and Coomassie-staining, comparing cleaved samples to uncleaved samples (LC3B: Figure 2-3, Lanes a and b; ATG7: Figure 2-4, Lanes a and b; ATG3: Figure 2-5, Lanes a and b).

The slurry was transferred into an empty Poly-Prep chromatography column prerinsed with wash buffer to collect flowthrough. When liquid completely passes through, another 1 column volume of wash buffer was applied on the top of resin. This second fraction containing residual protein was also collected. 


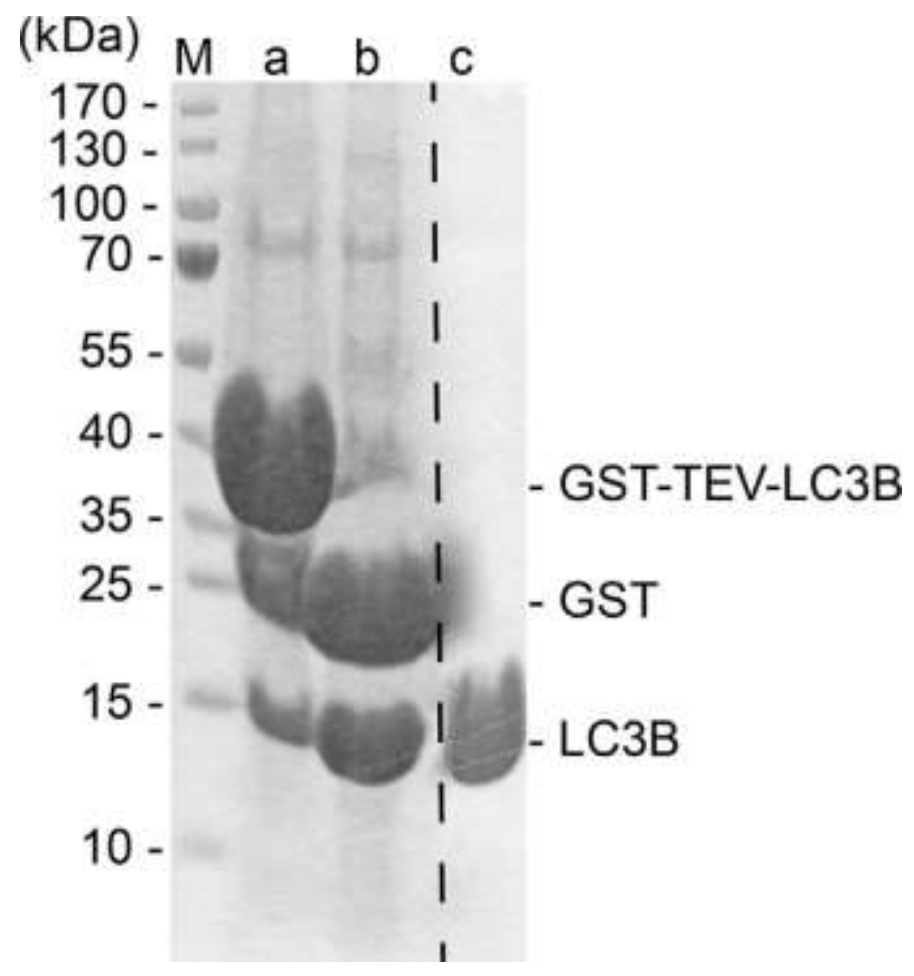

Figure 2-3. 15\% SDS-PAGE showing LC3B (1-120) at different steps of purification.

Lane $\mathrm{M}$ shows the molecular weight markers. Lane a shows constituents of bead slurry after binding GS4B resin. Lane b shows constituents of bead slurry after thrombin treatment. Lane c shows final purified LC3B (1-120) after gel filtration chromatography. 


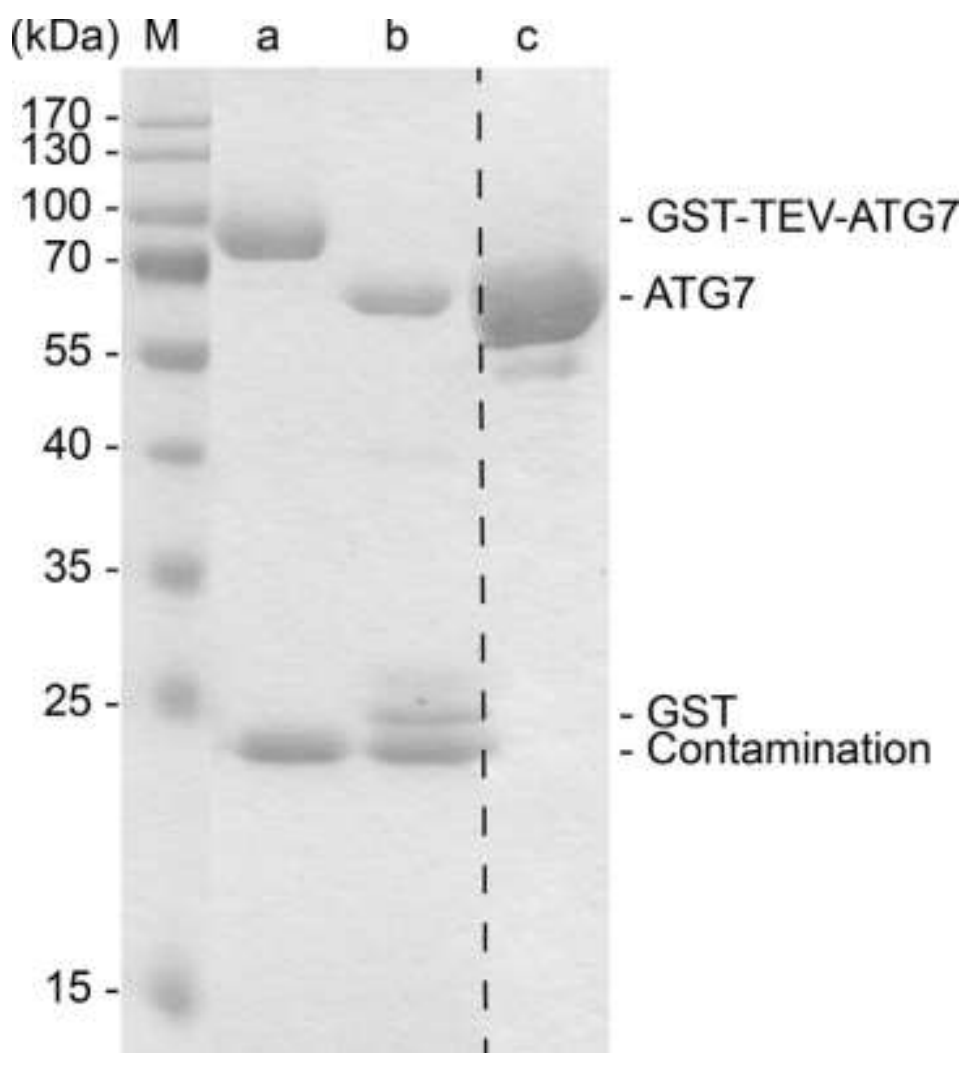

Figure 2-4. 15\% SDS-PAGE showing ATG7 at different steps of purification. Lane $\mathrm{M}$ shows the molecular weight markers. Lane a shows constituents of bead slurry after binding GS4B resin. Lane b shows constituents of bead slurry after TEV treatment. Lane c shows final purified ATG7 after gel filtration chromatography. 


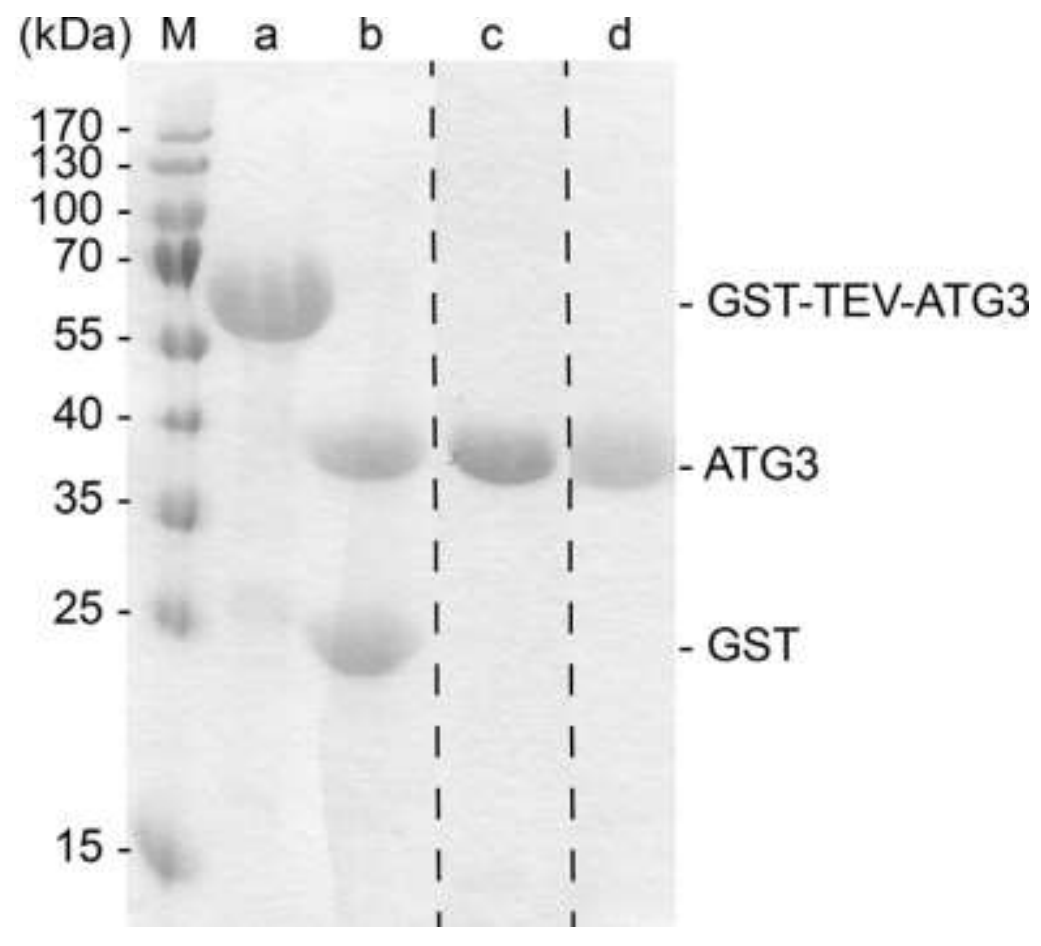

Figure 2-5. 15\% SDS-PAGE showing ATG3 at different steps of purification. LaneMshows the molecular weight markers. Lane a shows constituents of bead slurry after binding GS4B resin. Lane $b$ shows constituents of bead slurry after TEV treatment. Lane c shows purified ATG3 after anion ion exchange chromatography. Lane d shows final purified ATG3 after gel filtration chromatography. 


\section{Glutathione-mediated ATG12-ATG5-ATG16L1 elution from GS4B}

0.7 resin volume of GS4B elution buffer was gently applied on the top of resin surface while keeping stopcock closed. After a 10-min incubation, I opened the stopcock and collected the eluate in a tube. Steps above were repeat 6 times with elution fractions kept in separate tubes. GS4B elution buffer: wash buffer supplemented with $20 \mathrm{mM}$ glutathione (reduced, free acid) (EMD Millipore), $\mathrm{pH}$ adjusted by $\mathrm{NaOH}$ solution to the same as wash buffer.

Each eluted fraction were run on a 15\% SDS-PAGE gel to determine which fractions contain the majority of target protein. Desired fractions were then pooled.

\section{Nickel-affinity chromatography, imidazole-mediated elution from nickel beads, and TEV cleavage to obtain ATG12 (52-140)-ATG5-ATG16L1(1-69) complex}

After glutathione chromatography and glutathione mediated elution, the excessive GST-TEV-ATG16L1 (1-69) was further removed by nickel affinity chromatography.

HIS-Select Nickel Affinity Gel (Sigma-Aldrich) was washed 3 times with 10 column volumes of wash buffer in a chromatography column at $4{ }^{\circ} \mathrm{C}$. Then nickel-affinity chromatography was performed to enrich the ATG5-bound complex. The glutathione elution of GST-TEVATG12 (52-140)-His-TEV-ATG5-GST-TEV-ATG16L1 (1-69) was applied to the HIS-Select Nickel Affinity Gel beads and was incubated following the same procedure described above for glutathione-affinity chromatography. An imidazole elution was performed following steps described in glutathione elution using nickel bead elution buffer (wash buffer supplemented with $100 \mathrm{mM}$ imidazole $\mathrm{pH}$ 7.5). $10 \mu \mathrm{L}$ uncleaved sample was stored as a negative control to check cleavage efficiency.

TEV protease ( $2 \mathrm{mg}$ TEV per $100 \mathrm{mg}$ total protein) was added to the imidazole eluate. Then the eluate was dialyzed in wash buffer while performing cleavage overnight at $4^{\circ} \mathrm{C}$. Cleavage completeness was examined by SDS-PAGE, using a $15 \%$ acrylamide gel and Coomassie-staining (Figure 2-6, Lanes a and b). The cleaved GST and HisMBP tags were removed from ATG12 (52-140)-ATG5-ATG16L1 (1-69) complex by further cation ion exchange purification described below.

\section{Ion exchange chromatography on ATG3 and ATG12(52-140) ATG5-ATG16L1 (1- 69)}

Anion ion exchange was performed to tag-free ATG3 and cation ion exchange to tag-free ATG12 (52-140)-ATG5-ATG16L1 (1-69) accordingly to their isoelectric points, before ultimately purifying by gel filtration chromatography. This step is optional for LC3B and ATG7. 


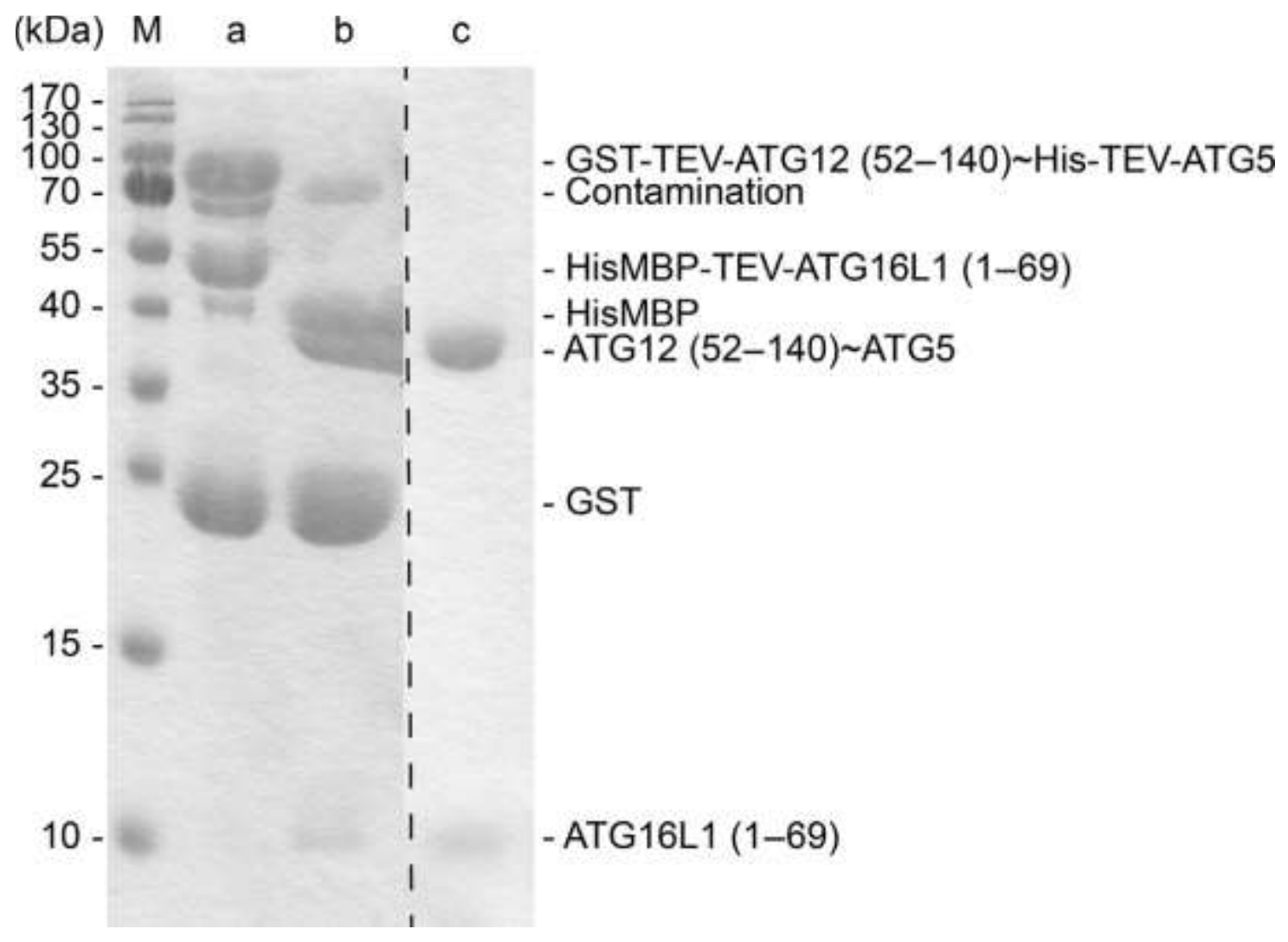

Figure 2-6. 15\% SDS-PAGE showing ATG12 (52-140)-ATG5-ATG16L1 (1-69) complex at different stages of purification.

Lane M shows the molecular weight markers. Lane a shows elution from nickel-affinity chromatography, capturing His- and His-MBP tags on ATG5 and ATG16L1 (1-69), respectively. This is the second step in the purification, following an initial purification by glutathione affinity chromatography to capture GST-tag on ATG12. Lane b shows product of TEV cleavage reaction preformed during dialysis. Lane c shows final purified ATG12 (52-140)_ATG5-ATG16L1 (1-69) complex after subsequent cation ion exchange and gel filtration chromatography. 
A 5 mL HiTrap SP (cation exchange) or a Q (anion exchange) HP column (GE Healthcare Life Sciences) was equilibrated on an AKTA FPLC (GE Healthcare Life Sciences) in 95\% buffer A with 5\% buffer B. Cation exchange buffer A: 25 mM HEPES pH 7.0, 5 mM DTT. Anion exchange buffer A: 25 mM Tris-HCl pH 8.0, 5 mM DTT. Buffer B corresponded to buffer A with additional $1 \mathrm{M} \mathrm{NaCl}$. The protein samples was diluted with buffer A to a final $\mathrm{NaCl}$ concentration of $50 \mathrm{mM}$. Samples were clarified by centrifugation at $4000 \mathrm{x}$ g for $5 \mathrm{~min}$ at $4^{\circ} \mathrm{C}$ before loading onto a FPLC system. If loading is through a sample pump, I would equilibrate the sample pump in buffer A. The ATG proteins described in this chapter can be concentrated to $10 \mathrm{mg} / \mathrm{mL}$ if desired to reduce volume prior to loading onto FPLC.

After equilibration, the sample was loaded onto column with a suggested loading rate of $2 \mathrm{~mL} / \mathrm{min}$. After loading, the column was washed by 5 column volumes of $95 \%$ buffer A with 5\% buffer B. After all unbound sample was washed out, I initiated gradient, raising buffer B proportion from 5\% to 50\% over 25 column volumes at 2 $\mathrm{mL} / \mathrm{min}$ flow rate, and collecting fractions with a volume of $2 \mathrm{~mL}$.

Peak fractions was examined by SDS-PAGE with Coomassie-stained 15\% acrylamide gels to determine which fractions contain the majority of the ATG proteins (the combined fractions of ATG3 is shown in Figure 2-5, Lane c; ATG12-ATG5ATG16L1 is not shown).

\section{Gel filtration chromatography for LC3B, ATG7, ATG3, and ATG12-ATG5- ATG16L1}

Superdex 200 10/300 GL columns (GE Healthcare Life Sciences) were used for all the ATG proteins described here, except for LC3B using a Superdex 75 10/300 GL column (GE Healthcare Life Sciences). Protein samples of LC3B and ATG7 used in this step were directly collected from protease cleavage, while ATG3 and ATG12-ATG5ATG16L1 were pre-purified by ion exchange.

A Superdex 75/200 column was equilibrated on an AKTA FPLC system with storage buffer ( $25 \mathrm{mM}$ Tris- $\mathrm{HCl}$ pH 7.6, $200 \mathrm{mM} \mathrm{NaCl}, 5 \mathrm{mM}$ DTT). $\mathrm{NaCl}$ concentration was icreased to $500 \mathrm{mM}$ in the buffer used for LC3B, or to $300 \mathrm{mM}$ in the buffer used for ATG12 (52-140)-ATG5-ATG16L1 (1-69).

Then the protein samples were concentrated by Amicon Ultra Centrifugal Filter Units (EMD Millipore) with appropriate molecular weight cutoff. Resulting concentrated protein samples were transferred to one or multiple $1.7 \mathrm{~mL}$ microcentrifuge tubes. Centrifugation was performed at $16,000 \mathrm{x}$ g for $10 \mathrm{~min}$ at $4^{\circ} \mathrm{C}$ to clarify samples before loading onto a FPLC system.

1-2 mL clarified protein sample was loaded onto a Superdex 200 column, followed by gel filtration run in storage buffer. The fractions were collected in $0.5 \mathrm{~mL}$ volume size. 
The peak fractions were examined by SDS-PAGE with Coomassie-stained 15\% acrylamide gels to determine which fractions contain the majority of the ATG proteins. Desired fractions were pooled (the pooled fractions of LC3B, ATG7, ATG3, and ATG12-ATG5-ATG16L1 are shown in Figure 2-3, Lane c; Figure 2-4, Lane c; Figure 2-5, Lane d; Figure 2-6, Lane c, respectively).

\section{Storing Purified Proteins}

Purified proteins was concentrated to approximately $10 \mathrm{mg} / \mathrm{mL}$ and stored in 20 $\mu \mathrm{L}$ aliquots at $-80^{\circ} \mathrm{C}$ after flash-freezing in liquid nitrogen. They can be thawed on ice for use and refreezed for storage up to twice to ensure protein activity.

\section{Concluding Remarks}

Proteins prepared as described earlier are eligible for multiple biochemical assays, including LC3B lipidation. In our experience, aliquots of purified ATG proteins stored at $-80^{\circ} \mathrm{C}$ are stable for up to a year, giving consistent results in assays for LC3B lipidation, formation of thioester bonded ATG7 LC3B and ATG3 LC3B intermediates, binding using BioLayer Interferometry (Octet Red, ForteBio) or isothermal titration calorimetry (ITC), and for structural studies using NMR and crystallography. 


\section{CHAPTER 3. A SWITCH ELEMENT IN THE AUTOPHAGY E2 ATG3 MEDIATES ALLOSTERIC REGULATION ACROSS THE LIPIDATION CASCADE ${ }^{2}$}

\section{Introduction}

The central enzyme in the Atg8 lipidation cascade is the E2, Atg3 (Klionsky, 2000; Wileman, 2013). Atg3 receives Atg8 from the E1 Atg7, to form a covalent, reactive Atg3 Atg8 intermediate linked by a thioester bond (referred to by $\sim$ ) between the C-terminus of Atg8 and the catalytic Cys of Atg3. Atg8 is then transferred from the Atg3 catalytic Cys to PE in a reaction catalyzed by a composite E3 enzyme (Fujita et al., 2008b)4/15/2020 10:13:00 AM containing another autophagy UBL, Atg12, conjugated to Atg5 (Hanada et al., 2007). In vitro, the yeast Atg12-Atg5 conjugate is sufficient to serve as an E3 enzyme activating lipidation of Atg8 (Hanada et al., 2007). Atg16 binding to lipids substantially enhances E3 activity (Lystad et al., 2019) and is required in vivo (Mizushima et al., 1999) to localize Atg12-Atg5 to the preautophagosomal structure that precedes autophagosome formation (Kim et al., 2001; Suzuki, 2001), through many mechanisms (Harada et al., 2019; Kaufmann et al., 2014; Romanov et al., 2012).

The prior structure of Atg3 from $S$. cerevisiae revealed a catalytic core domain (Atg $3^{\text {cat }}$ ) largely resembling E2s for canonical UBLs such as Ub, NEDD8 and SUMO, including a long central helix and two catalytic segments (Yamada et al., 2007) (Figure 3-1a). One segment harbors the catalytic Cys234, and the other displays Thr213, which also is essential for the lipidation reaction and corresponds to the canonical E2 catalytic Asn that plays a crucial structural role (Berndsen et al., 2013; Ichimura et al., 2000; Sakoh-Nakatogawa et al., 2013). In addition, Atg3 displays unique, functionallyimportant structures (Nath et al., 2014; Yamada et al., 2007; Yamaguchi et al., 2010): an $\mathrm{N}$-terminal amphipathic membrane-binding helix (N, residues 1-18); an intrinsicallydisordered flexible region (FR, residues 85-159); a handle region consisting of a helix and a disordered region (HR, residues 237-282) that contains an Atg8-interacting motif that is dispensable for the lipidation reaction but plays important roles in vivo; and a flexible extreme C-terminal extension (C, residues 299-310). While prior structural studies showed how Atg7 engages Atg $3^{\text {cat }}$ and the Atg $3^{\mathrm{FR}}$ for Atg8 transfer to Atg3 (Hong et al., 2011; Noda et al., 2011; Taherbhoy et al., 2011; Yamaguchi et al., 2012), less is understood about subsequent roles of the autophagy E3. A crystal structure showed how the human E3 enzyme recruits a peptide-like region from Atg3's FR, but this FR motif is not conserved across Atg3 sequences from many organisms, including yeasts (Metlagel et al., 2013). Furthermore, the structure lacked Atg3's catalytic domain, for which prior studies of cysteine accessibility in the yeast enzyme suggested that the autophagy E3 triggers a structural rearrangement that affects the orientation of the

\footnotetext{
${ }^{2}$ Reprinted from final submission with open access permission. Zheng, Y., Qiu, Y., Grace, C.R.R., Liu, X., Klionsky, D.J., and Schulman, B.A. (2019). A switch element in the autophagy E2 Atg3 mediates allosteric regulation across the lipidation cascade. Nature Communications 10, 1-14. https://doi-org.ezpprod1.hul.harvard.edu/10.1038/s41467-019-11435-y
} 


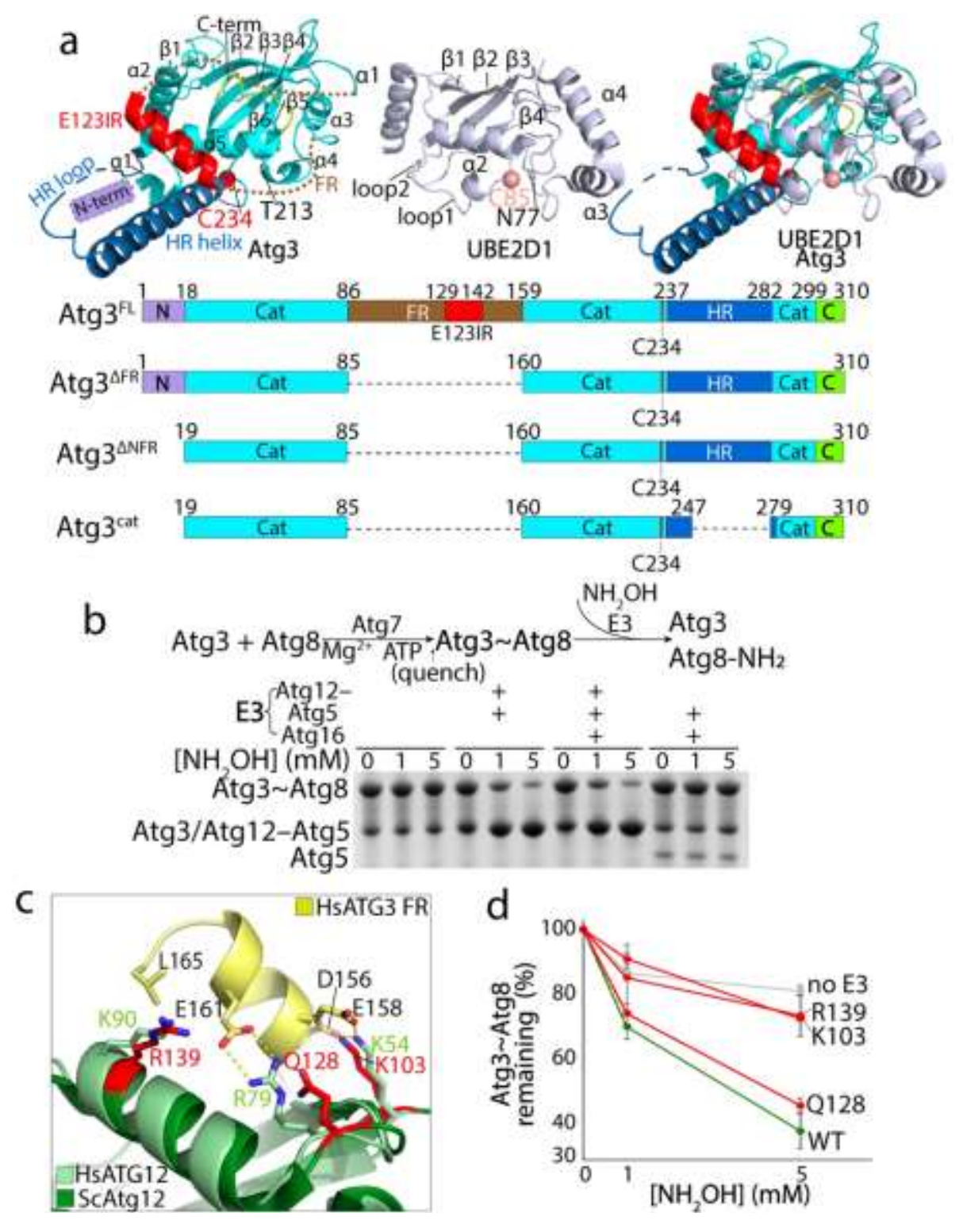

Figure 3-1. The Atg12-Atg5 module within the autophagy E3 activates intrinsic reactivity of the Atg3 Atg8 intermediate.

a, Structures of yeast Atg3 (left, PDB 2DYT), a ubiquitin E2 (middle, UBE2D1, PDB 4AP4), and their superimposition (right). Atg3 unique elements are indicated by colors shown in schematics of Atg3 constructs used in this study (below). b. Intrinsic reactivity of Atg3 Atg8 intermediate monitored by pulse-chase discharge to $\mathrm{NH}_{2} \mathrm{OH}$ in presence of indicated E3 variant. c, Close-up of structural superimposition of yeast Atg12-Atg5Atg16 (PDB 3W1S) and corresponding human complex with ATG3 ${ }^{\mathrm{FR}}$ (PDB 4NAW). Residues from yeast $\operatorname{Atg} 12$ corresponding to the human $\mathrm{ATG} 3^{\mathrm{FR}}$-binding site are shown as red sticks. d, Effects of mutations in minimal yeast E3 (Atg12-Atg5) on stimulating Atg3 Atg8 discharge, monitored by quantification of Atg3 Atg8 remaining after 2.5 minutes in pulse-chase assays using the scheme as in $b$, as a function of $\mathrm{NH}_{2} \mathrm{OH}$ concentration. (Error bar: STDEV, N = 3) Representative gel is shown in Supplemental Figure 3-2b. 
catalytic Cys234 relative to Thr213 (Sakoh-Nakatogawa et al., 2013). The molecular underpinnings for E3-dependent conformational activation remains elusive.

Here we use a hybrid structural approach integrating information from biochemistry, NMR, crystallography, and yeast genetics to identify a molecular basis for Atg3 allosteric activation. Overall, the data suggest that a distinct peptide-like element acts like a brace mediating intramolecular interactions with the Atg 3 catalytic domain to restrict the conformation of the active site and prevent reactivity toward errant nucleophiles, while the E3 elicits an unprecedented E2 activation mechanism by remotely binding to this brace to couple other E3 interactions with allosteric activation of active site mediating Atg8 lipidation.

\section{Methods}

\section{Protein sequence alignment} online.

ScAtg3 and HsATG3 protein sequence alignment are done by CLUSTALW

\section{Protein expression and purification}

Detailed methods for cloning, expressing and purifying yeast proteins Atg8, Atg3 (and their mutants or truncated versions) and Atg16 (residues 1-46) were described previously (Kaiser et al., 2013; Taherbhoy et al., 2011; Zheng et al., 2017). For Atg7 proteins, full-length Atg7 or a fragment containing residues 1-289 (NTD) was inserted into the pGEX-4T-1 vector following the Gibson Assembly protocol (Gibson et al., 2009), and transformed into BL21 competent E. coli; protein expression and purification protocols for Atg7 WT and mutants resemble those for Atg3 and Atg8. To obtain purified E3 complex, N-term 6X His-tagged Atg12 100-186 and untagged Atg5 1-284 were inserted into the two multiple cloning sites (MSCs) of pRSF-Duet-1 respectively, whereas Atg7 1-630 and Atg10 were inserted into the MSCs of pET-Duet-1. The two plasmids are co-transformed into BL21 competent E. coli to produce His-Atg12-Atg5. Bacteria culture were grown at $37^{\circ} \mathrm{C}$ to reach O.D. 1.5 , followed by $0.6 \mathrm{mM}$ IPTG induction at $23{ }^{\circ} \mathrm{C}$ overnight. Cells are harvested in $1 \mathrm{X}$ PBS, $400 \mathrm{mM} \mathrm{NaCl}$ and lysed by sonication. Ni-NTA resin (Qiagen) was incubated with lysate for 1 hour then washed 3 times with the same buffer. His-Atg12-Atg5 were eluted by buffer with $200 \mathrm{mM}$ imidazole and immediately purified by cation exchange chromatography on an S column (GE Healthcare). Purified his-Atg12-Atg5 were combined with Atg16 1-46 in a 1:2 molar ratio and further purified by size-exclusion chromatography on a Superdex-200 column (GE Healthcare) to harvest peak factions containing the final product of HisAtg12-Atg5-Atg16. 


\section{Crystallization and data collection}

$1 \mu \mathrm{L}$ purified $\operatorname{Atg} 3^{\Delta \mathrm{NFR}}$ protein sample was manually loaded onto a glass hangingdrop cover and overlaid with $1 \mu \mathrm{L}$ of precipitant solutions. Crystal was harvested from $100 \mathrm{mM}$ HEPES pH 7.2, $150 \mathrm{mM}$ L-malic acid, 9\% PEG3350. Crystals were allowed to grow at $4{ }^{\circ} \mathrm{C}$ for $5-7$ days to reach their maximum sizes. All crystals were harvested by loops with $25 \%$ glycerol in addition to growth solution, and stored in liquid nitrogen for 2 days until data collection.

\section{Data collection, processing, and structure refinement}

Data for Atg $3^{\triangle \mathrm{NFR}}$ was collected at Advanced Photon Source (APS) Ser-CAT beamline 22-BM. The Dataset was created from single crystals, and was processed and scaled in HKL2000. Phase was solved by molecular replacement. Search model for $\operatorname{Atg} 3^{\Delta N F R}$ was modified from Atg $3^{\mathrm{FL}}$ (PDB 2DYT) with Atg $3^{\mathrm{E} 123 \mathrm{IR}}$ manually removed. Model was built in Coot (Emsley et al., 2010), and refinements were primarily performed in Refmac (Murshudov et al., 1997) and finalized in Phenix (Adams et al., 2010).

\section{Simulated annealing omit map generation}

In order to avoid the model bias, we generated a simulated annealing omit map for the catalytic cysteine region. First, residue 231-237 in the final-refined model was deleted; then, the model with deletion was subjected to 3 rounds of refinement in Phenix ${ }^{62}$ with simulated annealing running from $5000 \mathrm{~K}$ to $300 \mathrm{~K}$ in 50 steps. Finally, the Fo-Fc map was shown at $3 \sigma$ level along with the deleted residues in the model.

\section{Atg3 C234 and Atg8 G116C disulfide crosslinking}

All the cysteines except C234 within Atg3 were mutated to alanines. $800 \mu \mathrm{M}$ Atg8 K26P C22V G116C was reduced by $20 \mathrm{mM}$ DTT, desalted into buffer 1 (20 mM HEPES pH 7.5, $50 \mathrm{mM} \mathrm{NaCl}$ ), immediately mixed with 1:1 v/v buffer 2 (20 mM HEPES $\mathrm{pH} 7.5,50 \mathrm{mM} \mathrm{NaCl}, 2.5 \mathrm{mM}$ 2,2'-dipyridyldisulfide (DPS). DPS stock was predissolved in a minimal amount of DMSO), incubated at room temperature (R.T.) for 20 min, and desalted again into buffer 1 . Meanwhile, $500 \mu \mathrm{M}$ Atg 3 was reduced by $20 \mathrm{mM}$ DTT and desalted into buffer 1, immediately mixed with DPS-Atg8 at a 1:2 molar ratio, and incubated at R.T for 1 hour. Crosslinking product was purified by anion exchange chromatography on Q column (GE Healthcare) and size-exclusion chromatography on a Superdex-200 column (GE Healthcare). 


\section{NMR spectroscopy}

All the experiments were conducted at $25{ }^{\circ} \mathrm{C}$ with protein samples dissolved in 20 $\mathrm{mM}$ MES pH 6.5, $100 \mathrm{mM} \mathrm{NaCl}$, and $10 \% \mathrm{v} / \mathrm{v} \mathrm{D}_{2} \mathrm{O}$ on either a Bruker Avance $600-\mathrm{MHz}$

or $700-\mathrm{MHz}$ or $800-\mathrm{MHz}$ spectrometer equipped with a $5-\mathrm{mm}$ triple resonance cryoprobe and a single-axis pulse field gradient. For resonance assignments of backbone atoms, 3D triple-resonance experiments were measure either with $0.4 \mathrm{mM}{ }^{13} \mathrm{C},{ }^{15} \mathrm{~N},{ }^{2} \mathrm{D}$ labeled or ${ }^{13} \mathrm{C},{ }^{15} \mathrm{~N}$-labeled $\mathrm{Atg} 33^{\mathrm{cat}}, 0.75 \mathrm{mM}{ }^{13} \mathrm{C},{ }^{15} \mathrm{~N}$-labeled $\mathrm{Atg} 3{ }^{\mathrm{FR}} 86-159$, and $0.4 \mathrm{mM}$ ${ }^{13} \mathrm{C},{ }^{15} \mathrm{~N}$-labeled Atg8 K26P, C33V, G116C. The backbone assignment of Atg $3{ }^{\text {cat }}$ was carried out using 3D HNCACB, HNcoCACB, HNCO, HNcaCO along with ${ }^{15} \mathrm{~N}$-resolved $\left[{ }^{1} \mathrm{H},{ }^{1} \mathrm{H}\right]$ NOESY spectra. The backbone of Atg $3{ }^{\mathrm{FR}}$ 86-159 fragment peptide was assigned using 3D HNCACB, CACBcoNH, HNCO, HNcaCO along with HNcocaNH spectra. The backbone assignment of Atg8 K26P, C33V, G116C was carried out using 3D HNCACB, HNcoCACB, HNCO, HNCA and HNcoCA along with ${ }^{15} \mathrm{~N}$-resolved $\left[{ }^{1} \mathrm{H},{ }^{1} \mathrm{H}\right] \mathrm{NOESY}$ spectra. Spectra were processed with Topspin 3.5 or nmrPipe and analyzed using CARA. All chemical shift perturbation analyses were done using $\left[{ }^{15} \mathrm{~N},{ }^{1} \mathrm{H}\right]$ TROSY spectra, and the perturbations were calculated using the formula $\operatorname{CSP}(\mathrm{ppm})=$ $\left((\delta \mathrm{H})^{\wedge} 2+0.2 *(\delta \mathrm{N})^{\wedge} 2\right)^{\wedge} 0.5$. Titration experiments were done using $0.1 \mathrm{mM}{ }^{15} \mathrm{~N}$-labeled Atg $3^{\mathrm{FR}} 86-159$ (or its I132D,L135D,I136D mutant) titrated with 0-150 $\mu \mathrm{M}$ Atg12-Atg5, $0-500$ or $0-625 \mu \mathrm{M} \mathrm{Atg} 3^{\Delta \mathrm{FR}}$, or 0 and $0.2 \mathrm{mM}$ unlabeled $\operatorname{Atg} 3^{\Delta \mathrm{FR}} \mathrm{C} 234 \sim \operatorname{Atg} 8 \mathrm{~K} 26 \mathrm{P}$, C33V, G116C disulfide complex; $0.1 \mathrm{mM}^{15} \mathrm{~N}$-labeled Atg $3^{\text {cat }}$ was titrated with 0 or 4.4 $\mathrm{mM}$ unlabeled $\mathrm{Atg} 3^{\mathrm{FR}} 123-147$. Also spectrum from $0.1 \mathrm{mM}{ }^{15} \mathrm{~N}$-labeled Atg8 was compared with $0.1 \mathrm{mM}$ disulfide complex of unlabeled Atg $3^{\text {cat }}$ cross-linked via C234 to ${ }^{15} \mathrm{~N}$-labeled Atg8 K26P, C33V, G116C. The CSP value is used in the titration plots to get the $\mathrm{Kd}$ values using the equation: $\mathrm{y}=(\mathrm{y} 0+\mathrm{x}) /(\mathrm{x}+\mathrm{Kd})$, where $\mathrm{x}$ is the concentration and $\mathrm{Kd}$ is the dissociation constant and $\mathrm{y} 0$ is a constant.

Since most of the resonances in $\operatorname{Atg} 3^{\mathrm{FR}}$ mutant [I132D, L135D, I136R] have the same chemical shifts as that of $A \operatorname{tg} 3^{\mathrm{FR}}$, the assignment of $\operatorname{Atg} 3^{\mathrm{FR}}$ was used to assign the resonances of $A \operatorname{tg} 3^{\mathrm{FR}}$ mutant. The assignment of the mutated residues and the neighboring ones that shifted the most ambiguously shifted to positions where new peaks appeared.

\section{Liposome preparation}

$2.5 \mathrm{mg}$ of $E$. coli polar lipid extract (Avanti) was dissolved in chloroform, solvent evaporated using a nitrogen stream, and re-dissolved in $250 \mu \mathrm{L} 20 \mathrm{mM} \mathrm{HEPES} \mathrm{pH} \mathrm{7.0,}$ $150 \mathrm{mM} \mathrm{NaCl}$ buffer. Product was passed through $100 \mathrm{~nm}$ nuclepore membrane (Whatman) installed in a mini extruder (Avanti) 15 times to obtain $10 \mathrm{mg} / \mathrm{mL}$ homogenized liposomes in solution. 


\section{In vitro pulse-chase assay for Atg3 Atg8 discharge to $\mathrm{NH}_{2} \mathrm{OH}$}

Shortly before the starting the experiment, Atg3 and mutants were reduced with $20 \mathrm{mM}$ DTT and desalted by Zeba columns (Thermo Fisher Scientific) into reaction buffer. In the pulse step, $5 \mu \mathrm{M}$ Atg7, $20 \mu \mathrm{M}$ Atg3, $40 \mu \mathrm{M}$ Atg8, $1 \mu \mathrm{M}$ ATP and $1 \mu \mathrm{M}$ $\mathrm{MgCl}_{2}$ were mixed in $25 \mu \mathrm{L} 20 \mathrm{mM}$ HEPES pH 7.0, $150 \mathrm{mM} \mathrm{NaCl}$ buffer. After 1 hour, pulse reactions were quenched by adding Apyrase. In chase step, $5 \mu \mathrm{L}$ of each pulse product was incubated with $5 \mu \mathrm{L}$ chase solution containing $5 \mu \mathrm{M}$ Atg12-Atg5-Atg16 and a course of $\mathrm{NH}_{2} \mathrm{OH}$ at R.T. for 2.5 minutes, or with liposome at a final concentration of 2 $\mathrm{mg} / \mathrm{mL}$ added in a timely manner. Samples were analyzed by $4 \%-12 \%$ Bis-Tris Protein Gels (Thermo Fisher Scientific) and stained with Coomassie Brilliant Blue. All raw data are available in the source data file.

\section{In vitro assay for Atg8 lipidation}

Atg3 and mutants were reduced and desalted as described in the pulse-chase assay. Reactions were performed at $30{ }^{\circ} \mathrm{C}$ in $25 \mu \mathrm{L}$ volumes of $20 \mathrm{mM}$ HEPES pH 7.0, $150 \mathrm{mM}$ buffer, with $1 \mu \mathrm{M}$ Atg7, $2 \mu \mathrm{M}$ Atg3, $5 \mu \mathrm{M}$ Atg8, $5 \mu \mathrm{M}$ Atg12-Atg5-Atg16, 1 $\mathrm{mM}$ ATP, $1 \mathrm{mM} \mathrm{MgCl}$ and $1 \mathrm{mg} / \mathrm{mL}$ liposome, and were monitored in timely manner. 6 $\mu \mathrm{L}$ of each reaction was mixed with $6 \mu \mathrm{L} 2 \mathrm{X}$ protein loading buffer. Samples were analyzed using a by $15 \%$ urea gel at $4{ }^{\circ} \mathrm{C}$ and stained with Coomassie Brilliant Blue. All raw data are available in the source data file.

\section{Yeast strains, vectors, media and growth conditions}

The XLY161 and the YCY131 (Cao et al., 2009) cells were transformed with pRS416 vectors harboring HA-tagged Atg3 (WT or mutants); the YCY131 cells were cotransformed with pRS414-Atg7-Atg10-Atg8 $\Delta$ R. For nutrient-rich conditions, yeast cells were grown in synthetic minimal (SMD; $0.67 \%$ yeast nitrogen base, $2 \%$ glucose, and auxotrophic amino acids and vitamins as needed) medium. For nitrogen starvation, cells were first cultured in appropriate SMD medium to mid-log phase; they were then shifted to the SD-N medium $(0.17 \%$ yeast nitrogen base without ammonium sulfate or amino acids, $2 \%$ glucose). The XLY161 cells were cultured in SMD-Ura to mid-log phase before they were shifted to SD-N medium for $2 \mathrm{~h}$; The YCY131 cells were cultured in SMD-Ura-Trp to mid-log phase before they were shifted to SD-N medium for $4 \mathrm{~h}$. All raw data are available in the source data file.

\section{Western blot}

$1 \mathrm{OD}$ of each of the yeast cultures was harvested and lysed in $50 \mu \mathrm{l}$ MURB (50 $\mathrm{mM}$ NaH2PO4, $\mathrm{pH}$ 7.0, $2.5 \mathrm{mM}$ MES, $\mathrm{pH}$ 7.0, 1\% SDS, $3 \mathrm{M}$ urea, 0.5\% $\beta$ mercaptoethanol, $1 \mathrm{mM} \mathrm{NaN} 3,0.2 \mu \mathrm{g} / \mu \mathrm{l}$ bromophenol blue) by vortexing them with glass beads at $4^{\circ} \mathrm{C}$ for $5 \mathrm{~min}$. The samples were then heated at $70^{\circ} \mathrm{C}$ for $10 \mathrm{~min}$ before 
they were loaded onto and run in SDS-PAGE gels, followed by transferring onto PVDF membranes. The membranes were blocked with 5\% skim milk, probed with primary antibodies (1:3000, Atg8; 1:10000, Dpm1; 1:3000, HA; and 1:10000, Pgk1), and then probed with horseradish peroxidase (HRP)-coupled secondary antibodies (1:10000, antirabbit and anti-mouse antibodies). Atg8 antiserum was generated by injecting purified yeast Atg8 peptides into rabbits; Dpm1 antibody is purchased from Molecular Probes (catalog\# A-6429); antibody to the HA epitope is from Sigma (catalog\# H3663); Pgk1 antibody is a generous gift from Dr. Jeremy Thorner, University of California, Berkeley. Goat anti-rabbit HRP sencondary antibody is from Fisher (catalog\# ICN55676), and rabbit anti-mouse HRP sencondary antibody is from Jackson ImmunoResearch (catalog\# 315-035-003). Chemiluminescence images were obtained by developing films in a dark room. The standard-sized protein bands from the protein ladder lanes on the blots were correspondingly hand-drawn using a pen onto the developed films. The hand-drawn protein ladders were not shown in the figures, but they can be found in the corresponding source files.

\section{Results}

\section{Autophagy E3 activates intrinsic reactivity of Atg3 Atg8}

E2 UB and E2 UBL intermediates are activated in distinct ways by different classes of E3 enzymes(Buetow and Huang, 2016; Cappadocia and Lima, 2018; Zheng and Shabek, 2017). Many E3s, for example RING and related E3s for UB, NEDD8 and SUMO, and SIM-containing SUMO E3s, allosterically promote noncovalent interactions between the E2 and UB (or UBL) moieties within the covalent intermediate. This is often monitored by an E3 increasing the susceptibility of an E2 UB or E2 UBL intermediate to nucleophilic attack by a nonspecific amine nucleophile (Saha and Deshaies, 2008). In contrast, HECT and RBR E3s use a different mechanism that depends on positioning the E2-bound UB for transfer to the E3 catalytic Cys. Although the autophagy E3 lacks homology to UB, NEDD8, and SUMO ligases, we asked whether there are common mechanistic features by testing biochemical activities of the relatively simplistic Atg8 pathway from $S$. cerevisiae. Potential E3-mediated stimulation of intrinsic activity of the Atg3 Atg8 intermediate was examined by adapting the pulse-chase assay format used to study canonical UB enzymes (Figure 3-1b). Briefly, a pulse-reaction catalyzed by Atg7 generated the thioester-linked Atg3 Atg8 intermediate. After quenching this reaction with apyrase, increasing concentrations of hydroxylamine were added (Figure 3-1b). Discharge of the Atg3 Atg8 intermediate to this nonspecific amine nucleophile was monitored by appearance of free Atg3 in SDS-PAGE. Whereas the Atg3 Atg8 intermediate was relatively stable on its own, addition of Atg12-Atg5 stimulated discharge. This activation was maintained in the presence of Atg16, while Atg5-Atg16 alone was insufficient (Figure 3-1b). Atg12-Atg5 is also known to be required for E3 activation of the lipidation reaction (Hanada et al., 2007; Lystad et al., 2019; SakohNakatogawa et al., 2013). Thus, within the composite autophagy E3 is a module that activates the intrinsic reactivity of the Atg3 Atg8 thioester-linkage. 
As a first step toward understanding the mechanism, we performed mutagenesis based on the prior structure showing that in the context of the human autophagy E3, Atg12 recruits a short peptide-like region of Atg3's FR (Metlagel et al., 2013). Although the FR sequences from human and yeast Atg3 are not conserved (Figure 3-2a), the structures of human and yeast Atg12-Atg5 superimpose well, including the $\operatorname{Atg} 3^{\mathrm{FR}}$ binding site (Figure 3-1c) (Noda et al., 2013; Otomo et al., 2013). Mutations in the corresponding surface of yeast Atg12-Atg5 not only impair Atg8 lipidation but also intrinsic activation of the Atg3 Atg8 intermediate (Figure 3-1d and Figure 3-2b,c). Thus, it seems probable that the FR from yeast Atg3 likewise contains an E3-binding element.

\section{Identification of an E3-binding element in yeast Atg3}

A multi-tiered approach was used to identify the Atg3 element determining E3 activation and binding. First, given that the corresponding E3-binding element from human Atg3 is located in the FR, a panel of Atg3 variants harboring individual or multiple alanine mutations in the FR were monitored for E3-dependent Atg3 Atg8 discharge (Figure 3-3a and Figure 3-4). Second, mutants hindering discharge were tested for relevance to autophagy, by testing for effects on E3-dependent Atg8 lipidation in vitro and in vivo (Figure 3-3b,c). Finally, NMR was used to define the $\operatorname{Atg} 3^{\mathrm{FR}}$ region mediating E3-binding. After resonances were assigned for a construct spanning the entire intrinsically-disordered $\mathrm{Atg} 3^{\mathrm{FR}}$ (residues 86-159), an unlabeled version of the Atg12Atg5 conjugate was added, and $\left[{ }^{15} \mathrm{~N},{ }^{1} \mathrm{H}\right]$ TROSY spectra were examined for chemical shift perturbation and/or line-broadening as an indicator of binding (Figure 3-5a,c). Although other residues in Atg3's FR contribute to activity (Figure 3-3a), the major region implicated by all assays spans from Ile129-Lys142 (Figure 3-3a-c and Figure 35a,c). For reasons described below, we termed this region $\operatorname{Atg} 3^{\text {E123IR }}$.

\section{Atg3's E3-binding element also binds E1 and E2}

Prior studies showed that the region corresponding to $\operatorname{Atg} 3^{\mathrm{E} 123 \mathrm{IR}}$ mediates Atg3 recruitment to the autophagy E1, Atg7 (Noda and Klionsky, 2008; Taherbhoy et al., 2011). In this upstream step of the conjugation cascade, Atg $3^{\text {E123IR }}$ binding to Atg7's Nterminal domain (Atg $7^{\mathrm{NTD}}$ ) anchors the complex, thereby increasing the local concentration for distal, transient interactions between Atg7's and Atg3's catalytic domains for Atg8 transfer between them (Kaiser et al., 2013) (Figure 3-6a). To further confirm that as in the human autophagy pathway (Ohashi and Otomo, 2015; Qiu et al., 2013), E1 and E3 binding to Atg3 is mutually exclusive for yeast enzymes, we examined the ability of the isolated NTD from Atg7 to compete with Atg12-Atg5-dependent activation of Atg3 Atg8. The wild-type Atg $7^{\text {NTD }}$ inhibits the E3-dependent reaction, whereas a version harboring a mutation (P283D) previously shown to impair Atg3 binding (Figure 3-6a) (Taherbhoy et al., 2011) does not show this inhibitory effect, consistent with mutually-exclusive binding (Figure 3-6b). 
a

ATG3_YEAST

ATG3_HUMAN

ATG3_yEAST

ATG3_HUMAN

ATG3_YEAST

ATG3 HUMAN

ATG3_YEAST

ATG3_HUMAN

ATG3_YEAST

ATG3_BUMAN

ATG3_YEAST

ATG3 HUMAN

$\mathrm{N}$-term

-MIRSTLSSWREYLTPITHKSTFLTTGQITPEEFVOAGDYLCHMFPTWKWNEESS MQNVINTVKGKALEVAEYLTPVLKESKFKETGVITPEEPVAAGDHLVHHCPTWQWATG-E

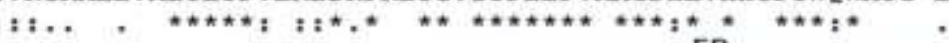
FR

DISYRDFLPKNKQFLIIRKVPCDKRAEOCVEVEGPDVIMKGFAEDGDEDDVLEYIGS--E ELKVKAYLPTGKOFLVTKNVPCYKRCKOMEYSDELEAI IEEDDGDGGWVDTYHNTGITGI

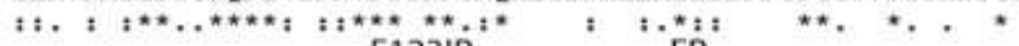
E123IR

TEHVQSTPAGGTKDSSIDDIDELIQDMEIKEEDENDDTEEFNAKGGLAKDMA TEAVKEITLENKDNIRLQDCSALCEEEEDEDEGEAADMEEYEESGLLETDEATLDTRKIV

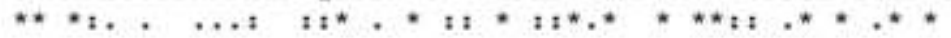

EACKAKTDAGGEDAILOTRTYDLYITYDKYYQTPRLWLFGYDEQRQPLTVEHMYEDISQD

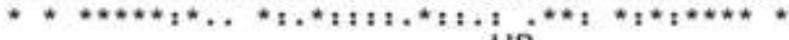
HR

YRTKTATIEKLPFYKNSVLSVS I HPCKHANVMK ILLDKVRVVRQRRRKELQEEQELDGVG HVKKTVTIENHPHLP-PPPMC SVHPCRHAEVMKK I IETVAEG -

$: * *, * * *: *, \quad *: * * * ; * * ; * \star * ;::, *$ HR C-term

DWEDLQDDIDDSLRVDQYLIVFLKFITSVTPSIQHDYTMEGW-

_._-_._-GGELGVHMYLLIFLKFVQAVIPTIEYDYTRHFTM $\ldots * * . * \star ;: t * * * * ;: ; * * t * ;: * * *$.

b

FR-interactive

Atg12 mutants

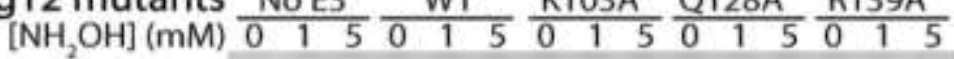

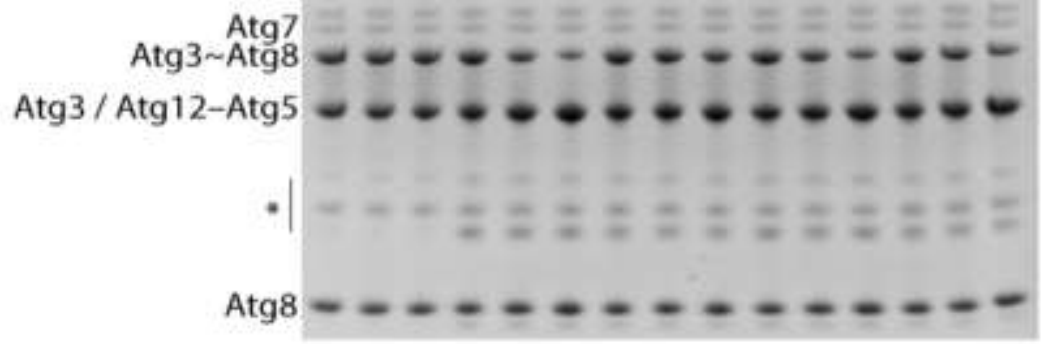

C

FR-interactive
Atg12 mutants
time (min)
$\begin{gathered}\text { Atg8 } 83 \\ \text { Atg } 8-P E\end{gathered}$

Figure 3-2. Lack of sequence conservation for Atg3 FR yet functional conservation of FR-binding site on Atg12 within Atg12-Atg5 E3 module.

a, Sequence alignment of Atg3 from S. cerevisiae (yeast) and H. sapiens (human). Elements in yeast Atg3 are labeled. $\mathbf{b}$, Representative gel image of 3 times repeated assays from Figure 3-1d. c, Effects of indicated Atg 12 Ala mutants in predicted FRbinding site from Atg12-Atg5 on Atg8 lipidation in vitro, in reactions with Atg7, Atg12Atg5-Atg16, and liposomes generated from E. coli polar lipids as a source PE, and detected by migration of Atg8 in Coommassie-stained SDS-PAGE gel. 

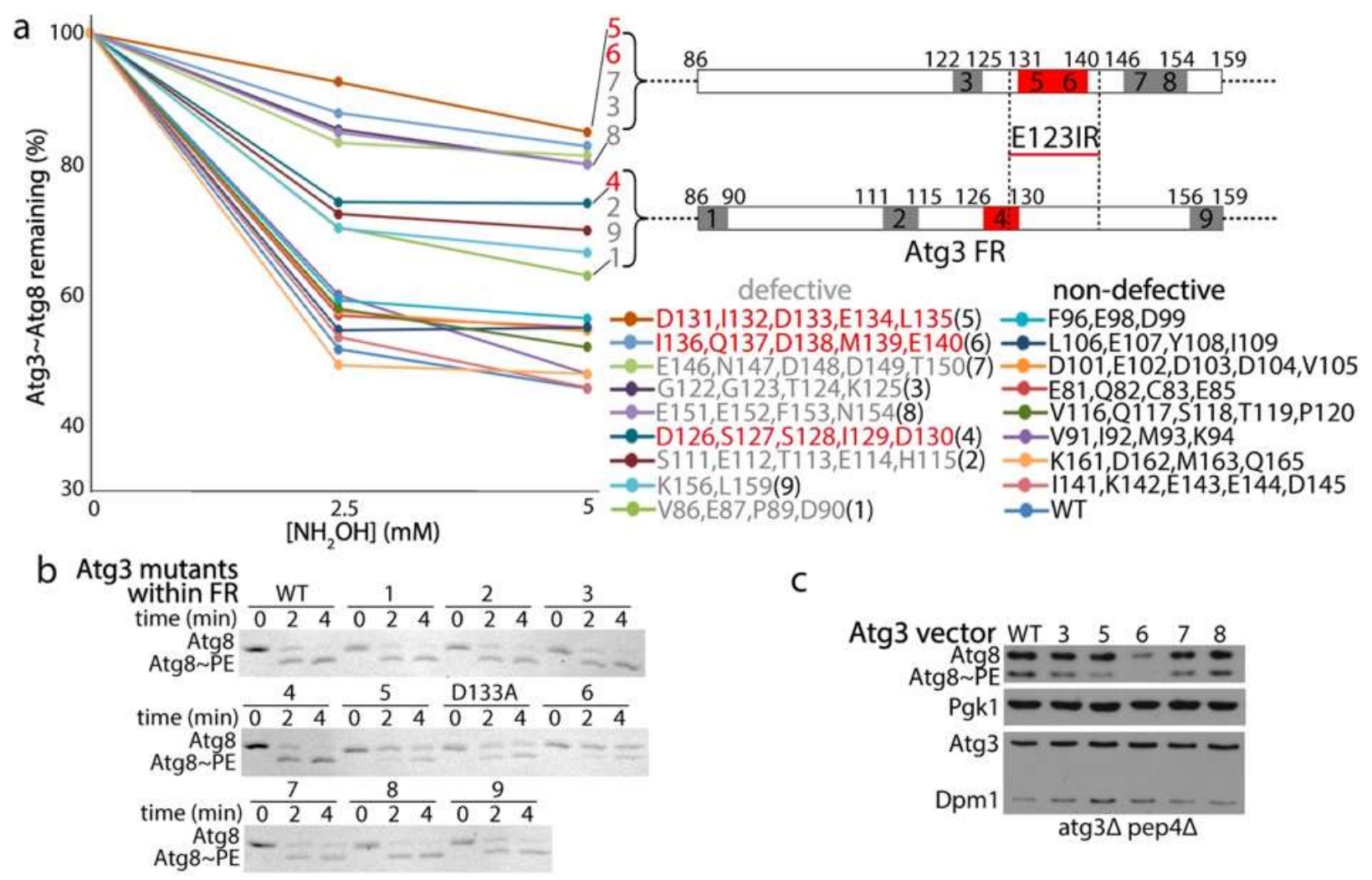

C

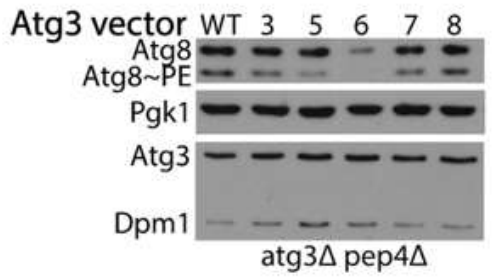

Figure 3-3. E123IR plays essential role in E3-dependent Atg8 ligation activity. a, Effects of indicated Ala mutants in Atg3's FR on E3 (Atg12-Atg5-Atg16) activation of Atg3 Atg8 intermediate, quantified as percent Atg3 Atg8 remaining in pulse-chase discharge to $\mathrm{NH}_{2} \mathrm{OH}$ over 2.5 minutes. Locations of most defective and moderately defective mutants are indicated on schematics, and those corresponding to $\mathrm{Atg} 3^{\mathrm{E} 123 \mathrm{IR}}$ are shown in red. b, Effects of indicated Ala mutants in Atg3's FR on Atg8 lipidation in vitro, in reactions with Atg7, Atg12-Atg5-Atg16, and liposomes generated from E. coli polar lipids as source of PE, and detected by migration of Atg8 in Coommassie-stained SDS-PAGE gel. c, Effects of indicated Ala mutants in Atg3's FR on Atg8 lipidation in vivo, as detected by western blot for Atg8 after 2 hours starvation of XLY161 $\operatorname{atg} 3 \Delta$ pep $4 \Delta$ strain of S. cerevisiae expressing either WT or mutant HA-tagged Atg3. Pgk1 is loading control for Atg8, Dpm1 is loading control for Atg3. 
Atg 3 mutants within FR

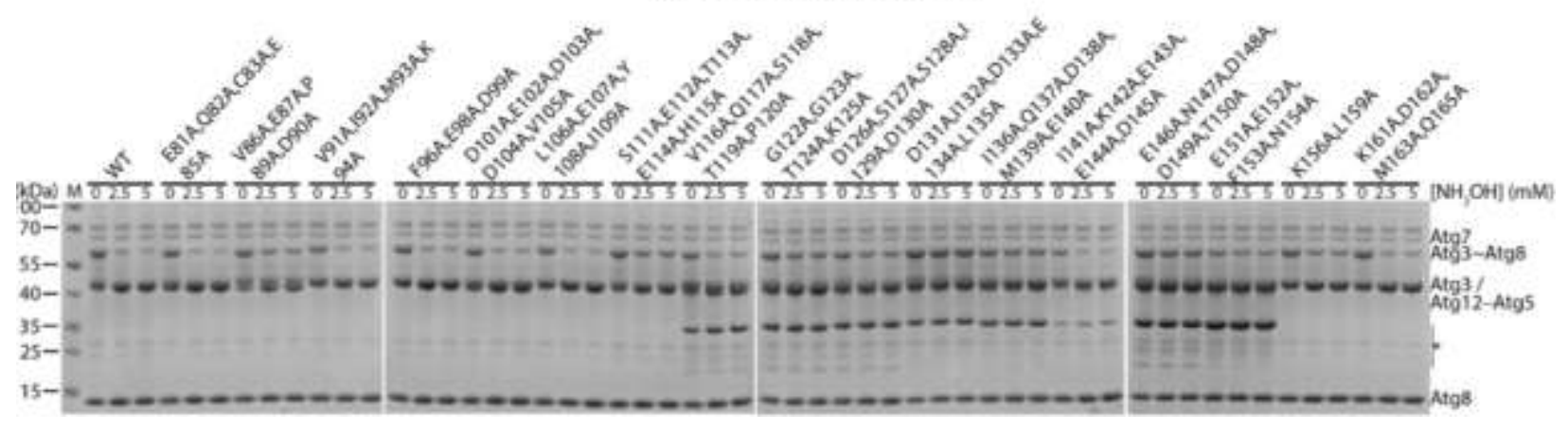

Figure 3-4. Alanine scan within FR of Atg3 for E3-dependent activation of Atg3 Atg8 intermediate.

Representative gel image of assay from Figure 3-3a.

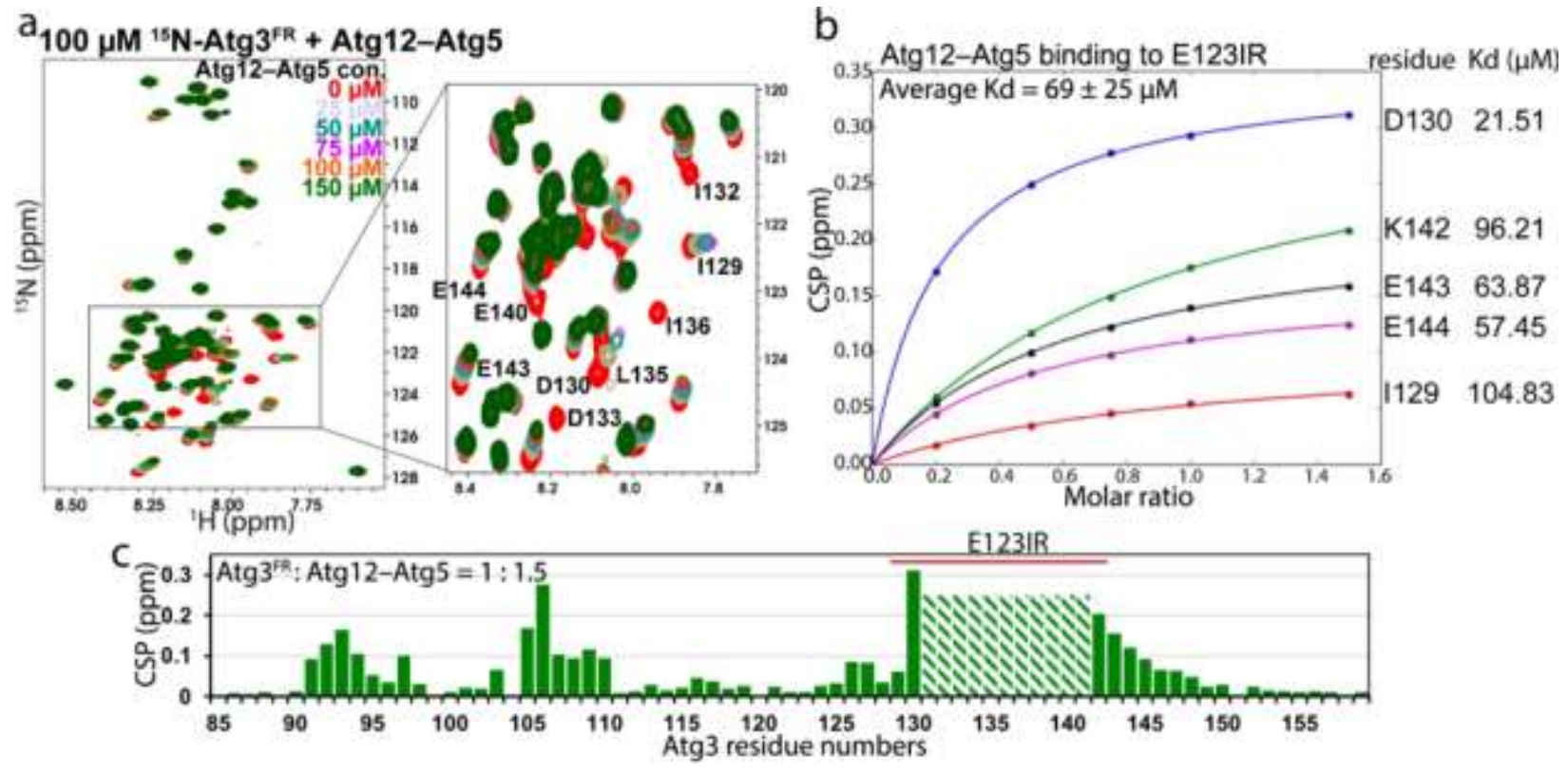

Figure 3-5. E123IR interacts with autophagy E3.

a, $\left[{ }^{15} \mathrm{~N},{ }^{1} \mathrm{H}\right]$ TROSY spectra of ${ }^{15} \mathrm{~N}$-labeled Atg $3{ }^{\mathrm{FR}}$ titrated with unlabeled Atg12-Atg5Atg16. b, Estimated binding affinities between E123IR residues and Atg12-Atg5, based on Chemical Shift Perturbations (CSPs) observed upon titrating increasing concentrations of Atg12-Atg5. c, Chemical shift perturbations plotted as a function of $\mathrm{Atg} 3^{\mathrm{FR}}$ residue numbers, with resonances showing intermediate exchange line broadening indicated by stripes. 
a

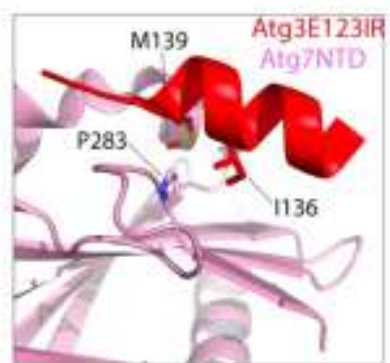

C
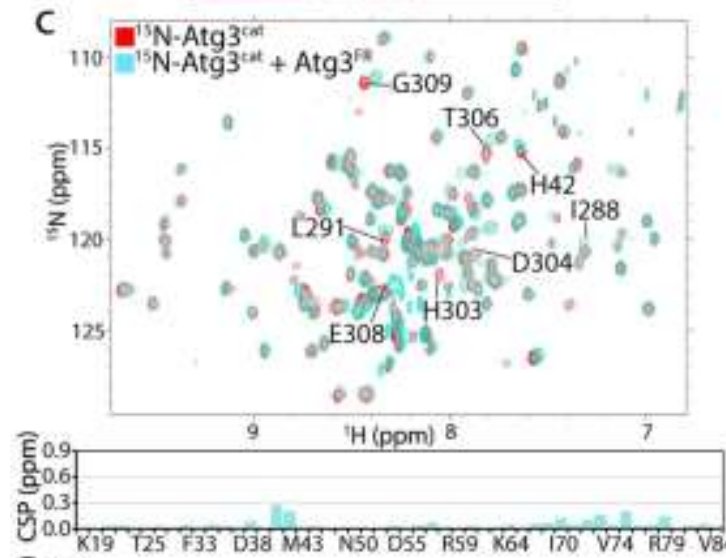

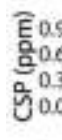

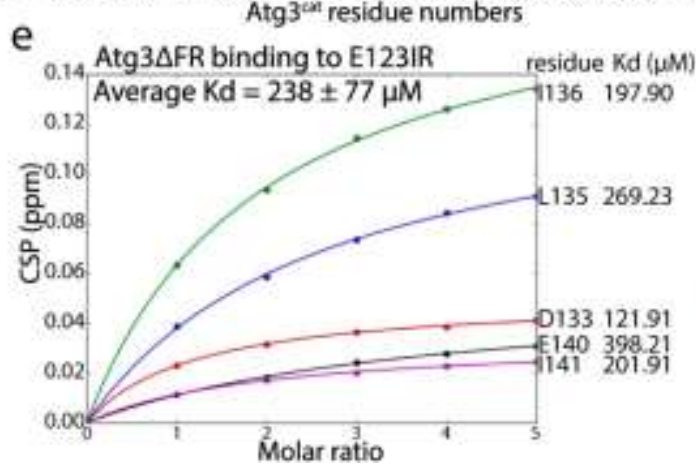

b

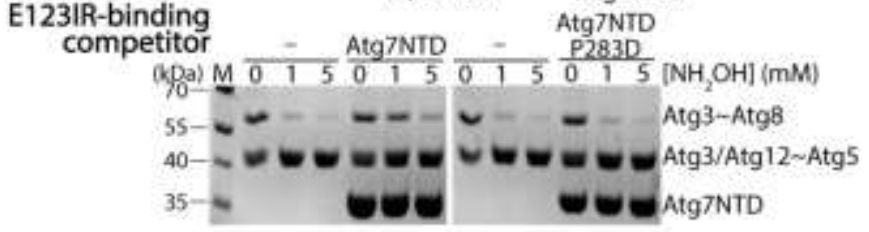

d $100 \mu M^{15} \mathrm{~N}-A \operatorname{tg} 3^{F R}+\operatorname{Atg} 3^{\text {IFR }}$
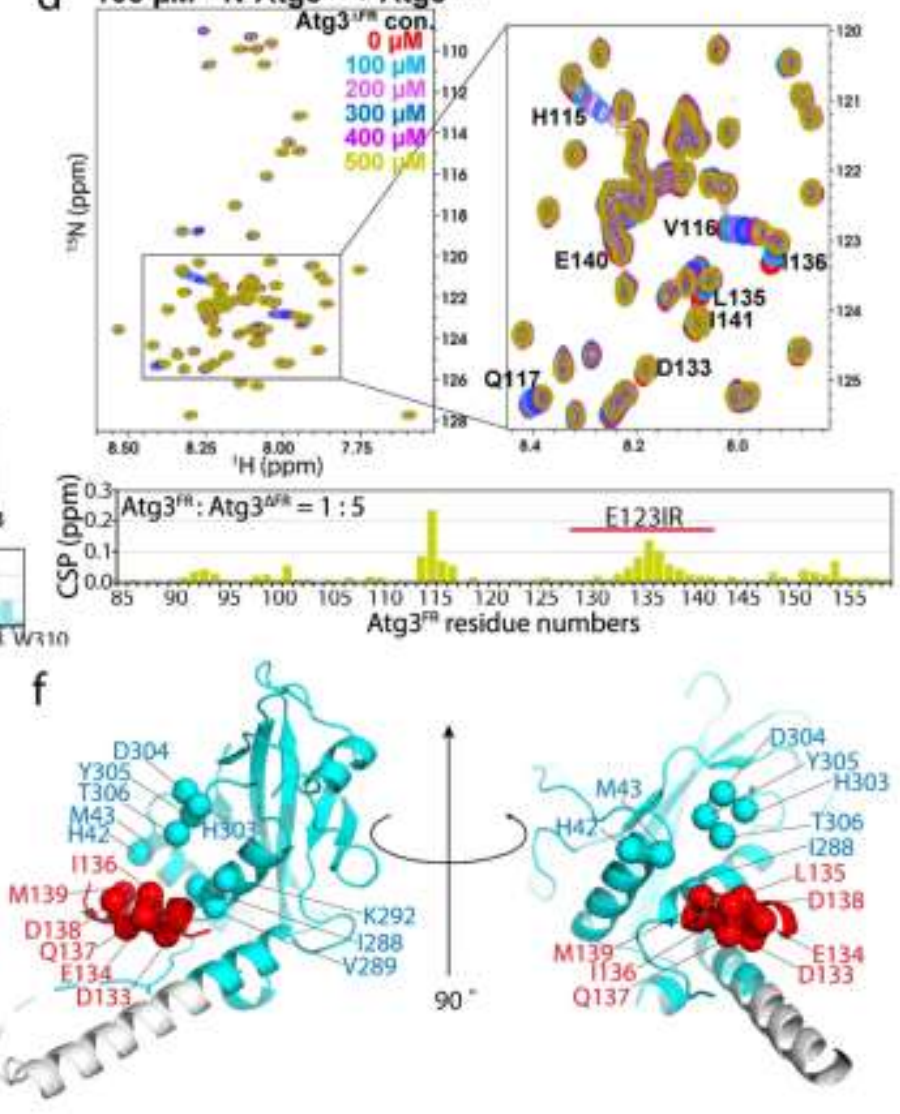

Figure 3-6. E123IR binds the E1 Atg7 and E2 core domain Atg3 ${ }^{\text {cat }}$.

a, Close-up showing interactions between Atg $3^{\text {E123IR }}$ and N-terminal domain (NTD) of

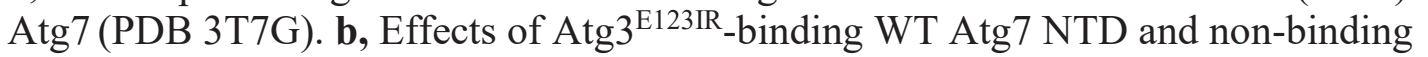
mutant (P283D) on E3-stimulated intrinsic reactivity of Atg3 Atg8 intermediate, as monitored by pulse-chase discharge to $\mathrm{NH}_{2} \mathrm{OH}$. $\mathbf{c},\left[{ }^{15} \mathrm{~N},{ }^{1} \mathrm{H}\right]$ TROSY spectra of ${ }^{15} \mathrm{~N}$ labeled Atg $3^{\text {cat }}$ alone (red) or in 1:44 mixture with unlabeled $\operatorname{Atg} 3^{\mathrm{FR}}$ (cyan), with Chemical Shift Perturbations (CSPs) plotted per residue below. d, $\left[{ }^{15} \mathrm{~N},{ }^{1} \mathrm{H}\right]$ TROSY spectra of ${ }^{15} \mathrm{~N}$-labeled $\operatorname{Atg} 3^{\mathrm{FR}}$ titrated with unlabeled $\operatorname{Atg} 3^{\Delta \mathrm{FR}}$, with representative $(1: 5$ molar ratio $\operatorname{Atg} 3^{\mathrm{FR}}$ vs $\operatorname{Atg} 3^{\Delta \mathrm{FR}}$ ) CSPs plotted per residue below. e, Estimated binding affinities between E123IR residues and $\operatorname{Atg} 3^{\Delta \mathrm{FR}}$, based on CSPs observed upon titrating increasing concentrations of $\mathrm{Atg} 3^{\Delta \mathrm{FR}}$. $\mathbf{f}$, Residues corresponding to greatest CSPs are shown as spheres on the structure. 
Intriguingly, this same E1 and E3-binding Atg3 ${ }^{\text {E123IR }}$ element was also found to contact the $\operatorname{Atg} 3^{\text {cat }}$ domain in the prior crystal structure of full-length Atg3 alone, although the interactions were previously attributable to crystal packing and not tested further (Figure 3-1a) (Yamada et al., 2007). To address the potential for bona fide interaction in solution, we wished to perform NMR. This first required generation of suitable fragments from Atg3, because in prior studies of the full-length protein, it was not possible to detect resonances from the catalytic domain due to dramatically different dynamic properties from the FR region (Yamada et al., 2007). Thus, the isolated Atg $3^{\text {cat }}$ domain was prepared (construct listed in Figure 3-1a), and resonances observed in $\left[{ }^{15} \mathrm{~N}\right.$ $\left.{ }^{1} \mathrm{H}\right]$ TROSY spectra were assigned (Figure 3-6c). Intriguingly, resonances corresponding to the active site loop (residues 232-236) were not assignable in the spectrum, potentially due to motions on the millisecond or intermediate exchange time scales.

$\left[{ }^{15} \mathrm{~N}-{ }^{1} \mathrm{H}\right]$ TROSY spectra obtained upon adding a synthetic peptide corresponding to the $A \operatorname{tg} 3^{\mathrm{FR}}$ element to ${ }^{15} \mathrm{~N}$-labeled $\mathrm{Atg} 3^{\mathrm{cat}}$ (Figure 3-6c), or upon adding an unlabeled version of $\operatorname{Atg} 3^{\Delta \mathrm{FR}}$ to ${ }^{15} \mathrm{~N}$-labeled Atg $3^{\mathrm{FR}}$ (Figure 3-6d), showed substantial chemical shift perturbations. In both titrations, the perturbed resonances correspond to the Atg $3^{\mathrm{FR}}$ and Atg $3^{\text {cat }}$ domain residues observed to interact in the crystal structure (Figure 3-6f). Consistent with the interactions, Introduction of mutations to hydrophobic residues I132, L135, and I136 significantly diminished the chemical shift perturbations within E123IR (Figure 3-7a), and further lower the E123IR-Atg $3^{\Delta \mathrm{FR}}$ binding affinity (Figure 3-7b). Thus, the NMR data confirmed the $\operatorname{Atg} 3^{\text {E123IR }}$ element as a bona fide $A \operatorname{tg} 3^{\text {cat }}$-binding element in solution. The term $\mathrm{Atg} 3^{\mathrm{E} 123 \mathrm{IR}}$ reflects this being an $\underline{\mathbf{E}} \mathbf{1}, \mathrm{E} \underline{\mathbf{2}}$, and E $\underline{\mathbf{3}}$ interacting region.

Compared to the interaction with Atg12-Atg5 (Figure 3-5b), we observed lower binding affinity between E123IR and $\mathrm{Atg} 3^{\mathrm{AR}}$ (Figure 3-6e). To gain insights into the structure of E123IR in its free form, we examined the $\Delta \delta C^{\alpha}-\Delta \delta C^{\beta}$ secondary chemical shifts, which show this element is not a helix in isolation (Figure 3-7c) (Marsh et al., 2006). It seems likely that a combination of different affinities for different partners, and intrinsic conformational plasticity could enable switching between E1, E2, and E3-bound states.

\section{Active site loop conformation in absence of $\operatorname{Atg}^{\text {E123IR }}$}

To illuminate roles of $\operatorname{Atg} 3^{\text {E123IR }}$-binding to the $A \operatorname{tg} 3^{\text {cat }}$, we determined a crystal structure lacking these interactions for comparison to the prior structure of full-length Atg3. The structure of a version of Atg3 lacking the N-terminal 18 residues and the FR region (Atg $3^{\triangle \mathrm{NFR}}$ ) (Figure 3-8a, Figure 3-9a, and Table 3-1), determined at $2.5 \AA$ resolution, superimposes well overall with that of full-length Atg3 (0.48 RMSD over Calphas) (Figure 3-8b). However, there are substantial differences in the catalytic Cys loop and adjacent regions. As described previously, in the structure of full-length Atg3, the catalytic Cys234 is sequestered in a pocket formed by side chains from the HR and two loops (Yamada et al., 2007). This orientation is catalytically incompetent, with the Cys distal from the structurally-important Thr213 and from two other side-chains 

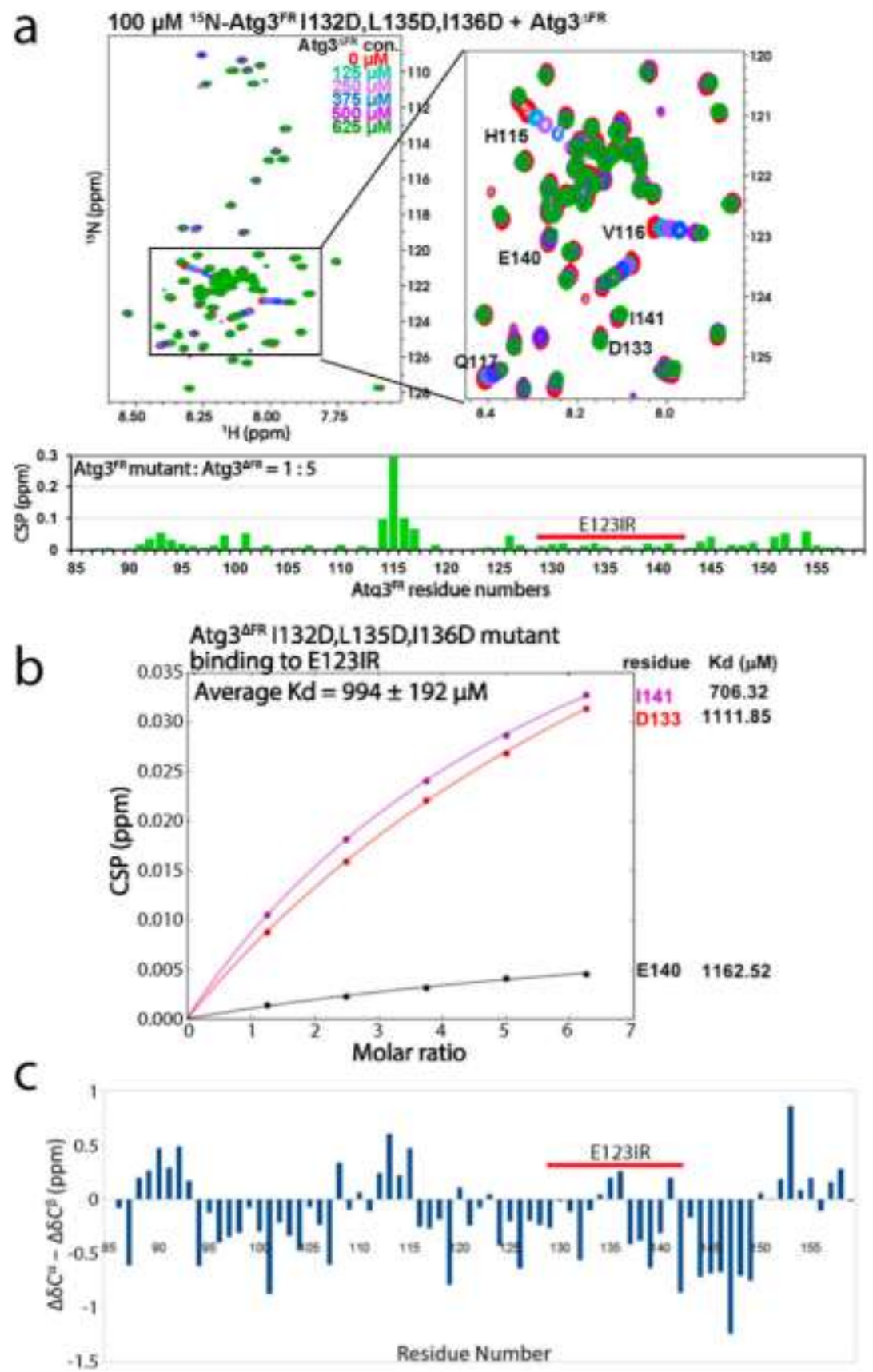

Figure 3-7. Mutations in E123IR hydrophobic residues significantly impair E123IR-Atg3 interaction.

a, $\left[15 \mathrm{~N},{ }^{1} \mathrm{H}\right]$ TROSY spectra of ${ }^{15} \mathrm{~N}$-labeled Atg $3^{\mathrm{FR}}$ titrated with increasing concentrations of unlabeled Atg $3^{\Delta \mathrm{FR}}$, with chemical shift perturbations (CSPs) plotted per residue shown below for one representative point in the titration (1:5 molar ratio Atg3FR mutant versus $\left.A \operatorname{tg} 3^{\Delta \mathrm{FR}}\right)$. b, Calculated binding affinities between $A \operatorname{tg} 3^{\Delta \mathrm{FR}}$ and residues in the I132D,L135D,I136D mutant version of Atg $3^{\mathrm{FR}}$, based on CSPs along the titration. $\mathbf{c}$, The $\Delta \delta C \alpha-\Delta \delta C \beta$ secondary chemical shifts of the Atg3FR peptide in its free form. 

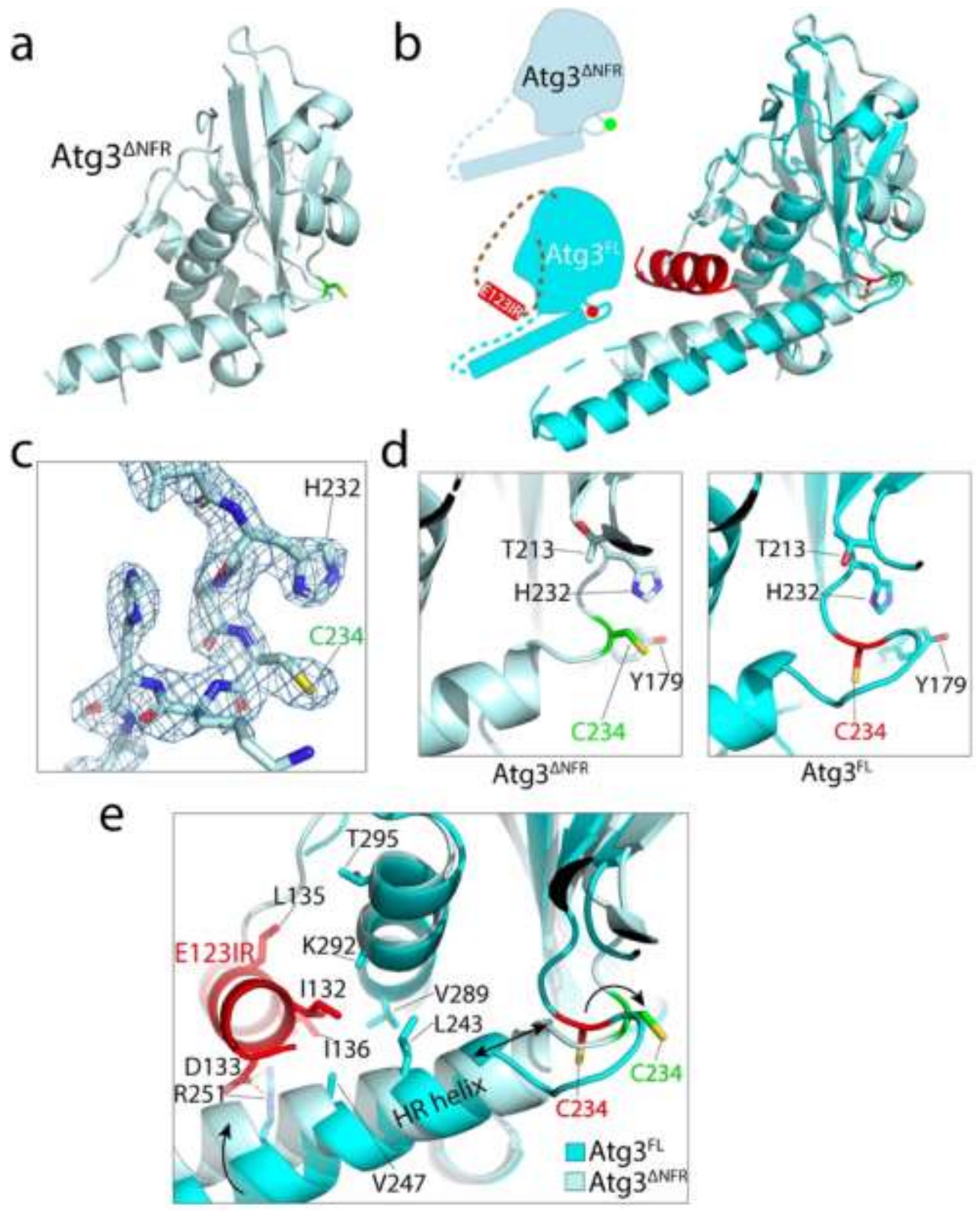

Figure 3-8. Conformational changes upon E123IR removal from Atg3. a, Crystal structure of Atg $3^{\triangle \mathrm{NFR}}$ (PDB 6OJJ, from this study). b, Superposition of Atg $3^{\triangle N F R}$ (PDB 6OJJ, light blue with catalytic Cys shown in green) with prior structure of $A \operatorname{tg} 3^{\mathrm{FL}}$ (cyan, PDB 2DYT) with E123IR and catalytic Cys shown in red, and differences highlighted in cartoons. c, Fo-Fc map shown at $3 \sigma$ after omitting the catalytic cysteine region (residues 231-237) of $A \operatorname{tg} 3^{\Delta \mathrm{NFR}}$ and performing simulated annealing. d, Close-ups of catalytic elements from $A \operatorname{tg} 3^{\Delta \mathrm{NFR}}$ and $\mathrm{Atg} 3^{\mathrm{FL}}$. e, Close-up superposition of Atg $3^{\Delta N F R}$ (light blue) and Atg $3^{\mathrm{FL}}$ (cyan) structures, showing interactions between Atg3's catalytic domain and E123IR, and conformational rearrangements upon E123IR dislocation. 

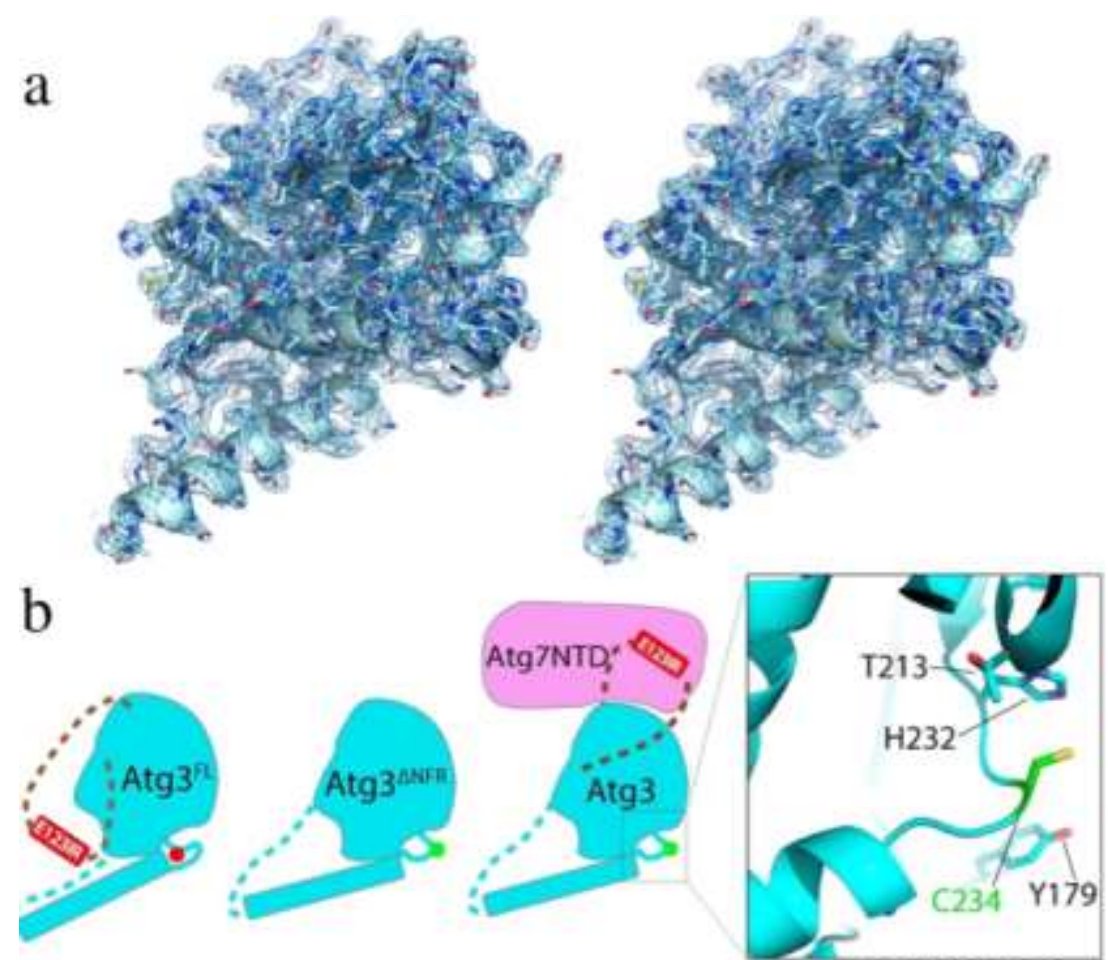

Atg7NTD-Atg3

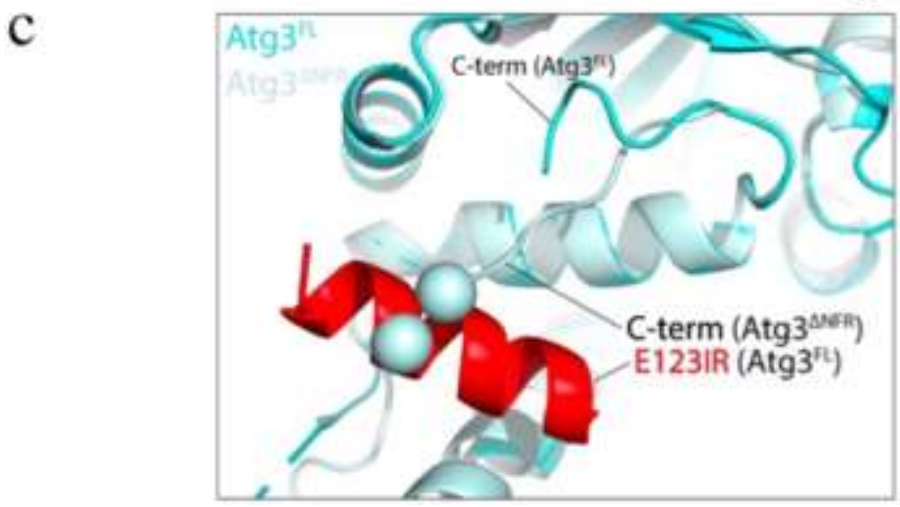

Figure 3-9. Structural remodeling of the Atg3 catalytic core upon displacement or removal of the E123IR element.

a, Stereo view of $A \operatorname{tg} 3^{\triangle \mathrm{NFR}}$ crystal structure in $2 \mathrm{Fo}-\mathrm{Fc}$ density map, $\sigma=1.0$. b , Schematics of structures of Atg3, with E123IR bound (from Atg $3^{\mathrm{FL}}$ PDB 2DYT), with E123IR removed in $\mathrm{Atg} 3^{\Delta \mathrm{NFR}}$ (this study, PDB 6OJJ), and with E123IR dislodged upon binding to Atg7 (PDB 4GSL), with the conformation in the Atg7-bound complex shown to the right for comparison to the structure with the E123IR deleted shown in Figure 3-8d. c, Superposition of the structure of $\operatorname{Atg} 3^{\Delta \mathrm{NFR}}$ (this study, light blue, PDB 6OJJ) and $\mathrm{Atg} 3^{\mathrm{FL}}$ (cyan PDB 2DYT) shows potential rearrangement of Atg3's extreme C-terminal element between E123IR-bound and -displaced forms of Atg3. Residues not visible in the prior structure are highlighted with spheres for alpha carbons. The conformation of Atg3's extreme C-terminal residues observed in the $\mathrm{Atg} 3^{\Delta \mathrm{NFR}}$ structure is incompatible with E123IR binding due to clashing, and is consistent with NMR chemical shift perturbations of this region detected by NMR in Figure 3-6. 
Table 3-1. Data collection and refinement statistics

\begin{tabular}{|c|c|}
\hline Parameters & $\begin{array}{c}\operatorname{Atg}^{\Delta N F R} \\
(\operatorname{ScAtg} 3 \Delta 1-18, \Delta 86-159)\end{array}$ \\
\hline \multicolumn{2}{|l|}{ Data collection } \\
\hline Space group & C 121 \\
\hline \multicolumn{2}{|l|}{ Cell dimensions } \\
\hline$a, b, c(\AA)$ & $92.078,44.557,66.2$ \\
\hline$\alpha, \beta, \gamma\left({ }^{\circ}\right)$ & $90,102.977,90$ \\
\hline Resolution $(\AA)$ & $30-2.40(2.44-2.40)^{*}$ \\
\hline $\mathrm{R}_{\text {merge }}$ & $0.050(0.766)$ \\
\hline$I / \sigma(I)$ & $7.9(1.1)$ \\
\hline$C C_{1 / 2}$ & $0.996(0.587)$ \\
\hline Completeness (\%) & $92.6(77.3)$ \\
\hline Redundancy & $3.4(2.8)$ \\
\hline \multicolumn{2}{|l|}{ Refinement } \\
\hline Resolution $(\AA)$ & $26.41-2.50$ \\
\hline No. reflections & 9090 \\
\hline $\mathrm{R}_{\text {work }} / \mathrm{R}_{\text {free }}$ & $0.2127 / 0.2427$ \\
\hline No. atoms & 1519 \\
\hline Protein & 1458 \\
\hline Water & 61 \\
\hline B factors $\left(\AA^{2}\right)$ & 51.20 \\
\hline Protein & 51.24 \\
\hline Water & 50.23 \\
\hline \multicolumn{2}{|l|}{ R.m.s. deviations } \\
\hline Bond lengths $(\AA)$ & 0.006 \\
\hline Bond angles $\left({ }^{\circ}\right)$ & 0.918 \\
\hline
\end{tabular}


(Tyr179 and His232) that are important for the lipidation reaction (Figure 3-8d, right) (Kaiser et al., 2013; Sakoh-Nakatogawa et al., 2013; Yamada et al., 2007). By contrast, in the absence of the Atg $3^{\text {E123IR }}$ element, Atg3's catalytic center is structurally rearranged into an activated conformation through a domino-like effect that ultimately results in remodeling a loop observed in full-length Atg3 into an additional helical turn at the Nterminus of the HR (Figure 3-8e); the remodeled helix cannot restrain the side-chain from catalytic Cys234, thereby allowing the alternative orientation where Cys234 is surrounded by additional key catalytic residues (Figure 3-8d, left). Moreover, it appears that the extreme C-terminal region of Atg3 also rearranges between the E123IR-bound and other states: this element undergoes a conformational change and additional $\mathrm{C}$ terminal residues are visible in the crystal structure of $A \operatorname{tg} 3^{\Delta \mathrm{NFR}}$ relative to the prior structure of full-length Atg3. In the conformation in Atg $3^{\Delta N F R}$ these residues would clash with the bound E123IR (Figure 3-9b,c), although their nearby location also suggests potential to positively interact with the E123IR in an alternative, presently unknown conformation. Indeed, resonances from these residues display large NMR chemical shift perturbations when ${ }^{15} \mathrm{~N}$ labeled $\mathrm{Atg} 3^{\text {cat }}$ is titrated with the unlabeled FR (Figure 3-6c).

\section{Mutants displacing $\operatorname{Atg}^{\mathrm{E} 123 I R}$ from $\operatorname{Atg}^{\text {cat }}$ activate ligation}

Interestingly, the activated orientation of the catalytic center was observed previously in structures of full length Atg3 when bound to Atg7 (Figure 3-9b) (Kaiser et al., 2013; Yamaguchi et al., 2012). Although interpreted as stemming from indirect Atg7 interactions with the backside of Atg3's catalytic domain (Kaiser et al., 2013; Yamaguchi et al., 2012), and/or from the high $\mathrm{pH}$ of the crystallization conditions (SakohNakatogawa et al., 2013), reinterpretation of the crystal structures with the knowledge that the Atg $3^{\text {E123IR }}$ element binds Atg3's catalytic domain in solution leads to a prediction: interactions with $\operatorname{Atg} 3^{\text {E123IR }}$ would allosterically restrict Atg3's catalytic domain. However, displacement of the Atg $3^{\text {E123IR }}$ element, either by deleting the FR domain (Figure 3-8d), or upon binding to the E1 Atg7 during formation of the Atg3 Atg8 intermediate (Figure 3-9b), or upon binding to the Atg12-Atg5 portion of E3 for the lipidation reaction (Sakoh-Nakatogawa et al., 2013), would enable the active conformation. Nonetheless, relevance for the lipidation would depend on the Atg $3^{\text {E123IR }}$ element binding in the context of an Atg3 Atg8 complex. We tested this concept by NMR. First, a stable proxy for Atg $3^{\Delta \mathrm{FR}} \sim \operatorname{Atg} 8$ was made with a disulfide bond between a Cys replacement for the C-terminal Atg8 residue and the catalytic Cys in Atg $3^{\Delta \mathrm{FR}}$ (Atg $3^{\Delta \mathrm{FR}}$-S-S-Atg8). Upon adding this to the ${ }^{15} \mathrm{~N}$-labeled Atg $3^{\mathrm{FR}}$, we observed the similar chemical shift perturbations in $\left[{ }^{15} \mathrm{~N},{ }^{1} \mathrm{H}\right]$ TROSY spectra of ${ }^{15} \mathrm{~N}$-labeled Atg $3{ }^{\mathrm{FR}}$ (Figure 3-10a) as for Atg3 alone (Figure 3-6d).

If binding to the $\operatorname{Atg} 3^{\text {E123IR }}$ would be inhibitory, then mutations in catalytic domain residues that mediate this interaction and that are not needed for other Atg3 functions should stimulate the lipidation reaction in the absence of E3. Therefore, based on the crystallographic (Figure 3-8e) and NMR data (Figure 3-6f), we generated several Ala substitutions designed to disrupt the autoinhibitory interactions as follows: Asp133, 


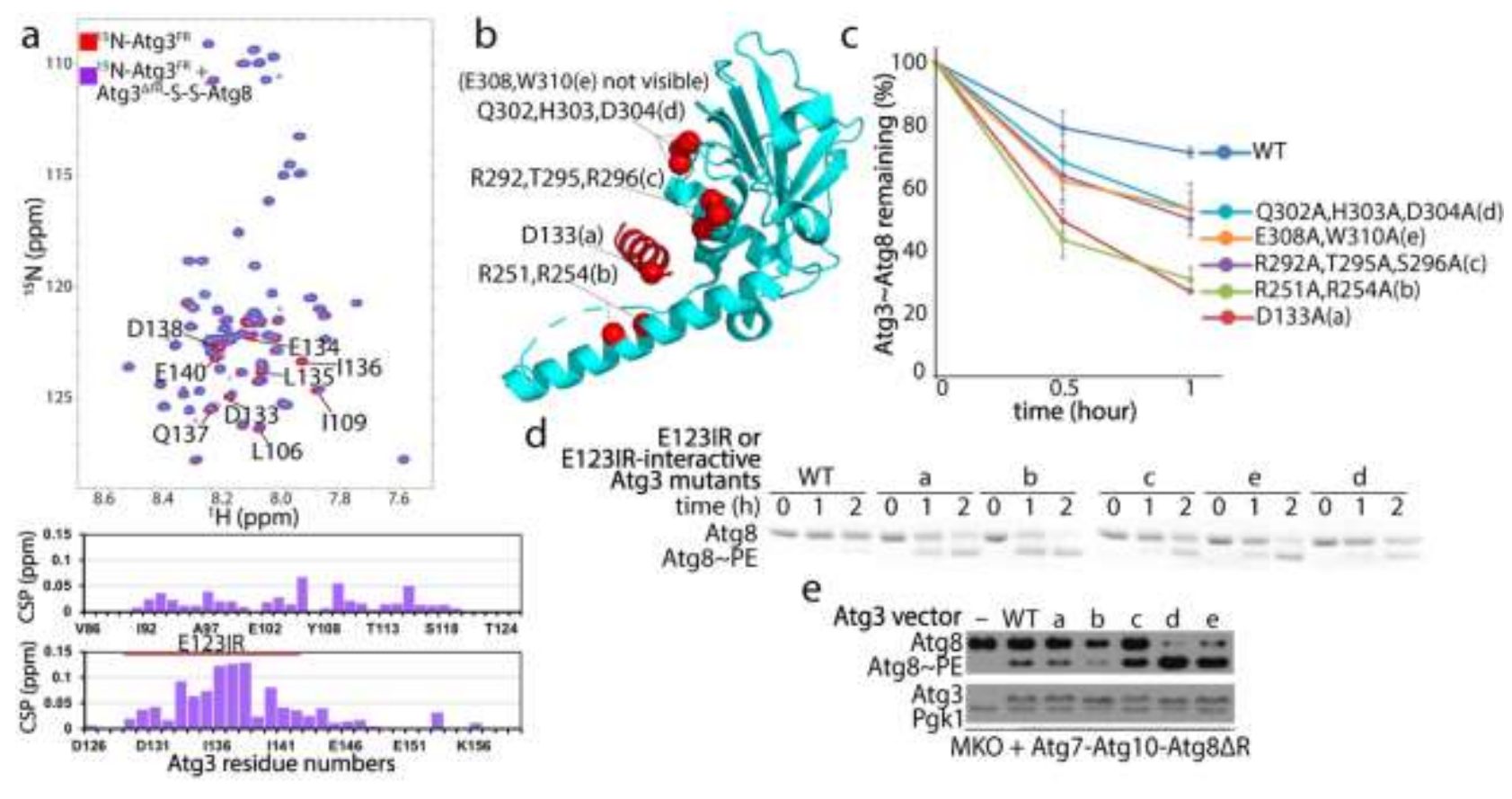

Figure 3-10. Mutations in E123IR-binding residues activate Atg3 Atg8 in the absence of $\mathrm{E} 3$ in vitro and in vivo.

a, $\left[{ }^{15} \mathrm{~N},{ }^{1} \mathrm{H}\right]$ TROSY spectra of ${ }^{15} \mathrm{~N}$-labeled $\mathrm{Atg} 33^{\mathrm{FR}}$ alone (red) or in 1:2 mixture with unlabeled, disulfide-bonded proxy for $\operatorname{Atg} 3^{\Delta \mathrm{FR}} \sim \operatorname{Atg} 8$ (purple), with Chemical Shift Perturbations (CSPs) per residue shown below. b, Locations of mutations a-e designed to impair interactions between Atg3's catalytic domain and E123IR shown on crystal structure of Atg3 (PDB 2DYT). c, Effects of indicated mutants in Atg3 catalytic domainE123IR interface on intrinsic E3-independent activity of Atg3 Atg8 intermediate.

Quantification is of WT or indicated mutant versions of Atg3 Atg8 remaining after 2.5 minutes as a function of $\mathrm{NH}_{2} \mathrm{OH}$ concentration in pulse-chase assays, without E3, repeated 3 times repeats average. (Error bar: STDEV, $\mathrm{N}=3$ ) Representative gel is shown in Figure 3-11. d, Effects of indicated mutants in Atg3 catalytic domain-E123IR interface on E3-independent Atg8 lipidation in vitro, in reactions with Atg7, Atg12Atg5-Atg16, and liposomes generated from E. coli polar lipids as a source PE, and detected by migration of Atg8 in Coommassie-stained SDS-PAGE gel. e, Effects of indicated mutants in Atg3 catalytic domain-E123IR interface on E3-independent Atg8 lipidation in vivo, as detected by western blot for Atg8 after 4 hours starvation of the YCY131 multi-Atg knockout strain of $S$. cerevisiae expressing Atg7, Atg10, Atg8 $\Delta \mathrm{R}$ (activated in absence of Atg4) and either WT or mutant HA-tagged Atg3. 
which is the only residue in the $A \operatorname{tg} 3^{\text {E123IR }}$ that does not bind Atg7 and thus could form an Atg3 Atg8 intermediate, interacts with the HR in full-length Atg3; Arg251 and Arg254 in the HR; Lys292, Thr295, and Ser296 in the Atg $3^{\text {cat }}$ domain; and Gln302, His303, and Asp304 or Glu308 and Trp310 at the extreme C-terminus (Figure 3-10b). These mutations mimicked effects of adding E3 to the in vitro reactions lacking E3: relative to wild-type Atg3, they activate the Atg3 Atg8 thioester-bonded intermediate (Figure 3-10c and Figure 3-11) and the lipidation reaction (Figure 3-10d), but are either not affected or defective for E3-dependent activity (Figure 3-3b and Figure 3-12c). The defect for one mutant in E3-dependent activity can be explained due to its location in the E123IR (D133A), but other mutants do not map to a region presently known to be required for activity, although they may reflect an activated conformation of the Atg3 Atg8 intermediate. If this were the case, then the activation observed in the absence of E3 may even be lower than theoretically possible.

Notably, increased Atg3 Atg8 discharge was not observed upon adding liposomes together with the isolated $\operatorname{Atg} 7^{\mathrm{NTD}}$ that like E3 also binds the $\operatorname{Atg} 3^{\mathrm{E} 123 \mathrm{R}}$ (Figure 3-11). Thus, releasing the $\mathrm{Atg} 3^{\mathrm{E} 123 \mathrm{IR}}$ from the $\mathrm{Atg} 3^{\text {cat }}$ domain may not be sufficient for activating the lipidation activity of the Atg3 Atg8 intermediate. A potential difference between binding of the Atg $7^{\text {NTD }}$ versus Atg12-Atg5, or the effects of E3mimicking mutations, would be if the latter alter internal structural dynamics of the Atg3 Atg8 intermediate so as to increase susceptibility to nucleophilic attack.

To test the effects of the activating mutants in vivo, it is necessary to examine Atg8 lipidation in the absence of the proteins comprising the E3. We also wished to minimize confounding effects of Atg8, Atg3, and the Atg12-Atg5-Atg16 complexes interacting with many proteins associated with autophagy and non-autophagy-related pathways. Thus, we generated a minimal system for examining Atg8 lipidation in vivo, with a plasmid expressing Atg7, HA-tagged versions of Atg3, and the processed form of Atg8 in a yeast strain in which these and 21 other autophagy (Atg) genes have been deleted (MKO) (Cao et al., 2009). Atg8 lipidation was markedly increased with the majority of the mutants (Figure 3-10e), which is remarkable given the complexity of events required for the reaction and the large number of other proteins that interact with Atg3 and Atg8 in vivo.

\section{Extensive surfaces are required for Atg3 Atg8 activation}

To identify the portions of Atg8 and the Atg3 catalytic domain contributing to the activated state, we performed mutagenesis. E3-dependent discharge was monitored for 28 mutant versions of Atg8 and 27 mutant versions of Atg3, each having one to four Ala replacements for surface residues (Figure 3-12a,b and Figure 3-13a,b). The Atg8 mutations together probed the majority of its surface, while Atg3 mutations encompass the catalytic domain that is structurally homologous to canonical E2s and the short Cterminal extension. A strikingly large fraction of the mutants affected activation of Atg8 discharge. Notably, the majority of the most defective mutants were also defective for 


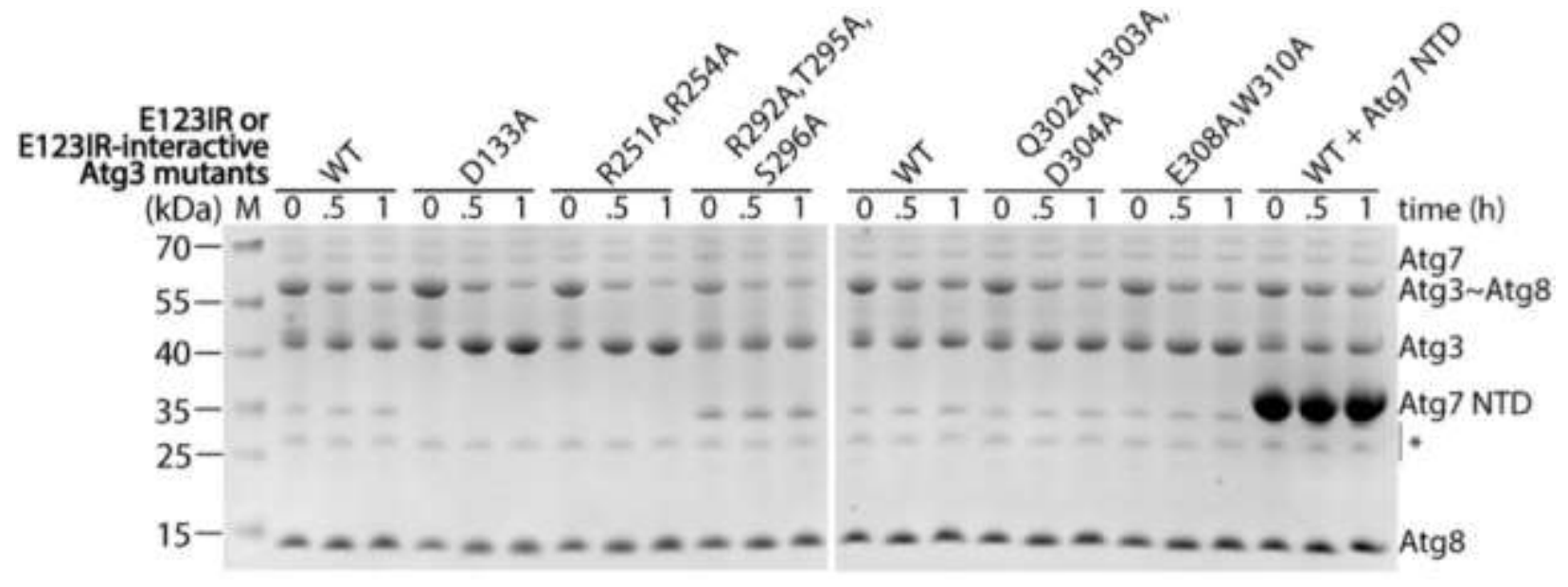

Figure 3-11. Mutations in interface between Atg3's E123IR element and catalytic domain activate the Atg3 Atg8 intermediate.

Representative gel image of assay from Figure 3-10c. 

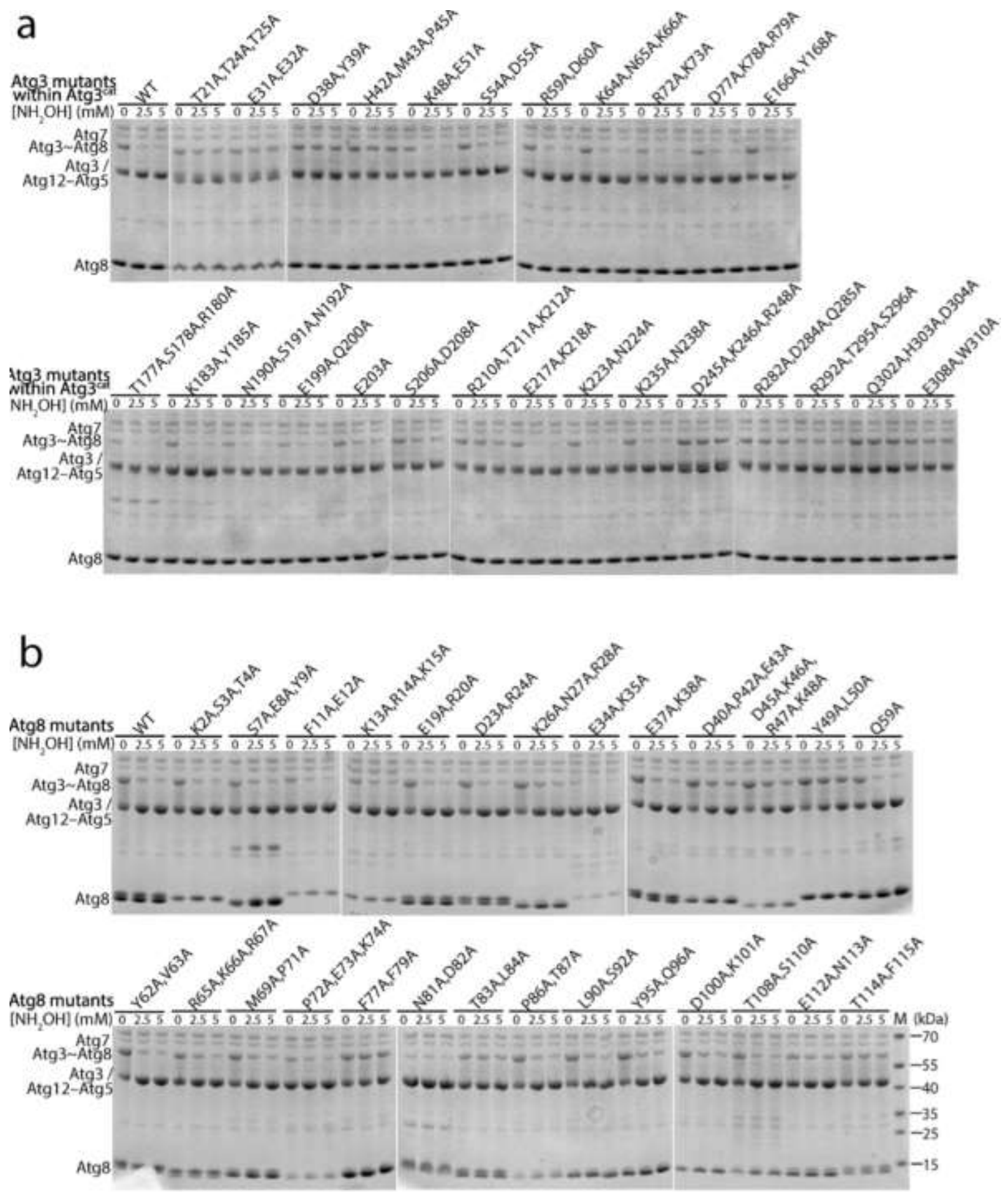

Figure 3-12. Alanine scan within Atg3cat and Atg8 by $\mathrm{NH}_{2} \mathrm{OH}$ discharge assays. a, Representative gel image of assay from Figure 3-13a. b, Representative gel image of assay from Figure 3-13b. 


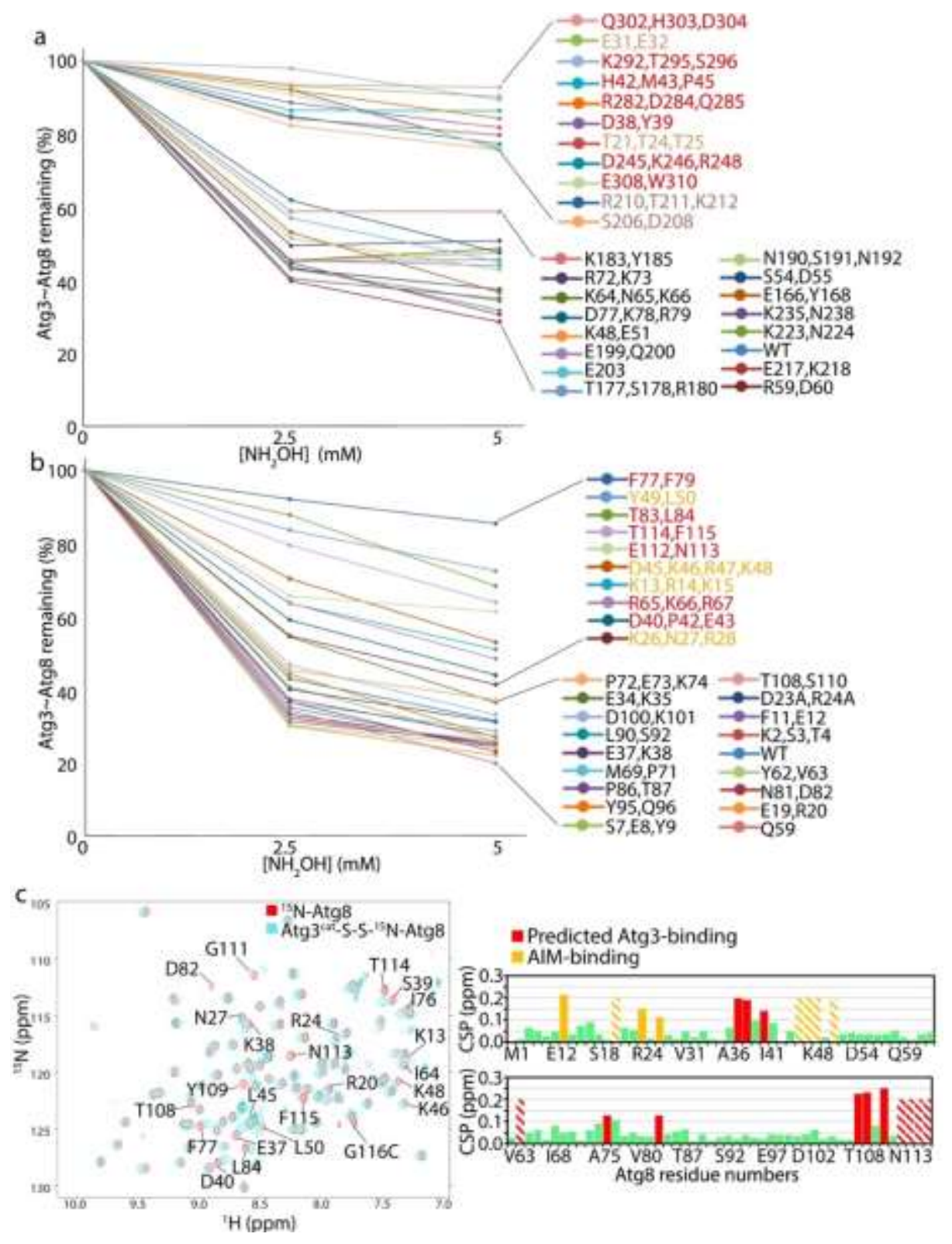

Figure 3-13. Extensive surfaces of Atg8 and the Atg3 catalytic domain are required for activation of the Atg3 Atg8 intermediate.

a, E3-dependent activation of Atg3 Atg8 intermediate testing roles of indicated surfaces through multiple-Ala scanning mutagenesis over Atg3's catalytic domain. Quantification is of WT or indicated mutant versions of Atg3 Atg8 remaining after 2.5 minutes as a function of $\mathrm{NH}_{2} \mathrm{OH}$ concentration in pulse-chase assays, with Atg12-Atg5-Atg16 as E3. b, E3-dependent activation of Atg3 Atg8 intermediate testing roles of indicated surfaces through multiple-Ala scanning mutagenesis over Atg8, performed as in a. c, Effects of covalent Atg3 complex formation with Atg8, as detected by comparing $\left.{ }^{15} \mathrm{~N},{ }^{1} \mathrm{H}\right] \mathrm{TROSY}$ spectra of ${ }^{15} \mathrm{~N}$-labeled Atg8 G116C (red) alone and disulfide-bonded complex with $\operatorname{Atg} 3^{\text {cat }}$ as a proxy for Atg $3^{\text {cat }} \sim \operatorname{Atg} 8$ intermediate (cyan), with chemical shift perturbations per Atg8 residue shown below. Stripes indicate resonances with line-broadening due to intermediate exchange. 
Atg8 lipidation in vitro (Figure 3-14a,b) and in vivo (Figure 3-14c). For Atg3, the defective mutants map to a large continuous surface encompassing Atg3's $\alpha 1-$, HR-, and $\alpha 5$ - (large central), $\alpha 4$ - (adjacent to the catalytic center) helices, and C-terminal extension (Figure 3-15b). For Atg8, the mutations map to two major surfaces. One corresponds to the canonical UBL hydrophobic patch and C-terminus, and the other to the Atg8-specific groove that binds AIM/LIR sequences in partner proteins (Figure 3-15c).

We hypothesized that some of the mutational defects could reflect impaired interactions between Atg 8 and Atg3 in the covalent intermediate. Thus, we performed NMR considering that prior studies of intrinsically active E2 UB intermediates showed chemical shift perturbations indicating noncovalent interactions between the covalently linked E2 and UB moieties. Thus, we compared $\left[{ }^{15} \mathrm{~N},{ }^{1} \mathrm{H}\right]$ TROSY spectra for ${ }^{15} \mathrm{~N}$-labled Atg8 harboring a $\mathrm{C}$-terminal Cys alone versus in a disulfide-bonded complex linked to the active site of unlabeled $\operatorname{Atg} 3^{\text {cat }}-$ i.e., in a stable proxy for the $\operatorname{Atg} 3^{\text {cat }} \sim \operatorname{Atg} 8$ intermediate (Figure 3-13c).

The striking similarity between the locations of Atg8 NMR resonances shifting upon covalent complex formation with Atg3 (Figure 3-15c), and of mutants impairing E3-activated discharge from Atg3 (Figure 3-15b) raise the possibility of noncovalent interaction in a so-called closed conformation. We used structural modeling to consider potential parallels between the activated Atg3 Atg8 intermediate and a RING E3-E2 UB complex as representative. First, the structure of Atg3 was superimposed with the homologous region of the E2 UBE2D1 in the intermediate, and then the UB-fold domain of Atg8 was docked onto the linked UB (Figure 3-15a). Next, the locations of mutations impairing activity, and NMR chemical shift perturbations in the Atg $3^{\text {cat }} \sim \operatorname{Atg} 8$ complex, were analyzed, and found to be in striking concordance with the corresponding interface residues in canonical E2 UB intermediates (Figure 3-15b,c,d). The notable exceptions were the AIM/LIR docking site in Atg8, Atg3 residues corresponding to E3-binding regions of canonical E2s, and the extreme C-terminus of Atg3. These regions are not shared between Atg3 and canonical E2s, and the latter region is not fully visible in the crystal structures presumably due to potential to adopt different conformations.

Nonetheless, the differences for the autophagy-specific regions that are observed between the crystal structures (Figure 3-9c), and in NMR chemical shift perturbations (Figure 3-6c), for the E123IR-bound and other forms of the Atg3 catalytic domain are consistent with roles in allosteric regulation. Indeed, in using the $A \operatorname{tg} 3^{\triangle \mathrm{NFR}}$ crystal structure for the structural model, the C-terminal residues of Atg3 approach the docked Atg8. On this basis, it is tempting to speculate that this sequence, Met-Glu-Gly-Trp, would adopt a reverse AIM/LIR motif anchoring Atg8 to Atg3 in a closed conformation. Concordantly, mutation of these residues has amongst the most deleterious effects (Figure 3-13a and Figure 3-12a, 3-14a), although future studies will be required to visualize the structure of the E3-activated Atg3 Atg8 intermediate. 

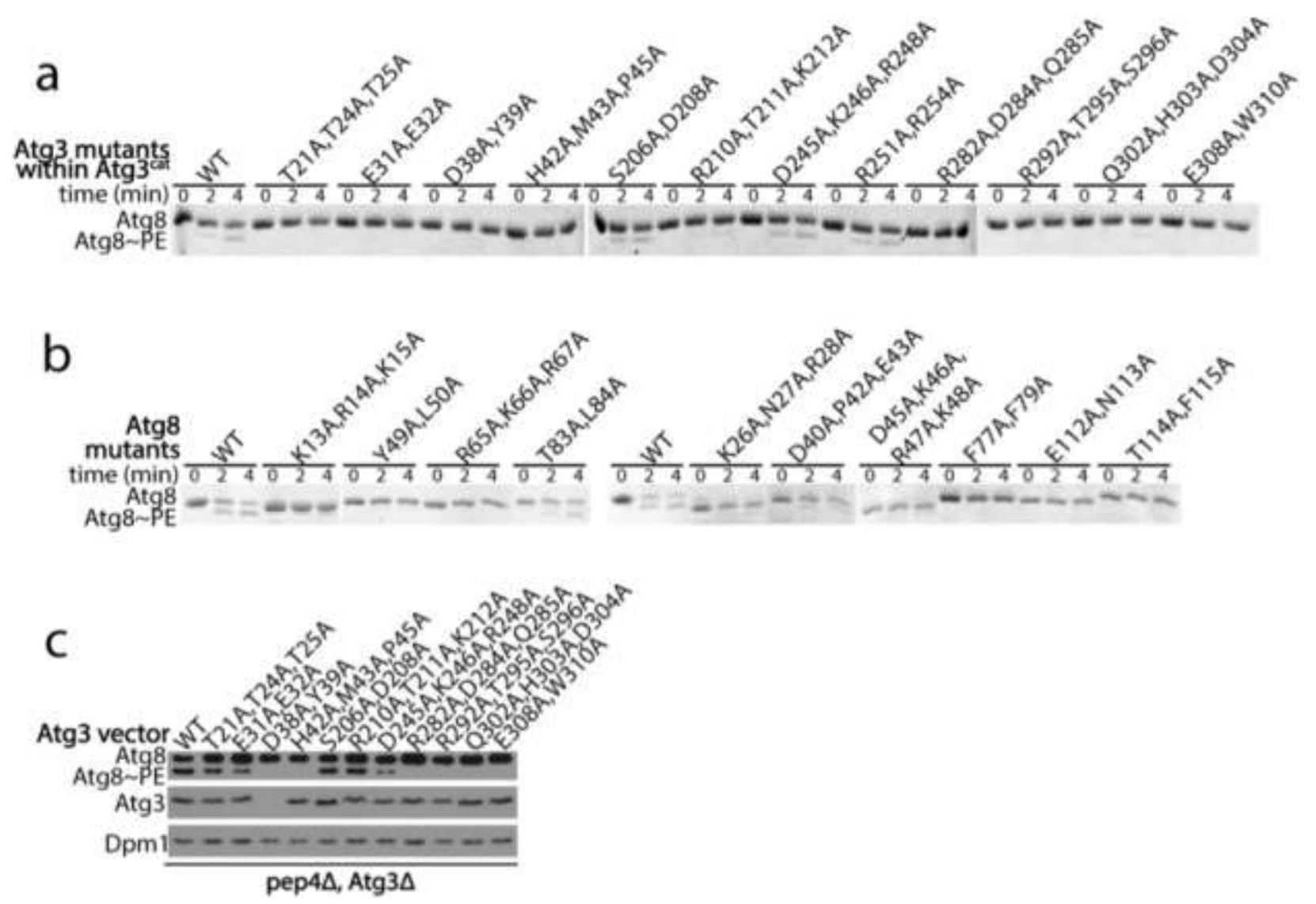

Figure 3-14. Alanine mutants within Atg3cat and Atg8 examined by Atg8lipidation assays in vitro and in vivo.

a, Effects of indicated mutants defective in a on E3-dependent Atg8 lipidation in vitro, in reactions with Atg7, Atg12-Atg5-Atg16, and liposomes generated from E. coli polar lipids as a source PE, and detected by migration of Atg8 in Coommassie-stained SDSPAGE gel. b, Effects of indicated mutants defective in b on E3-dependent Atg8 lipidation in vitro, in reactions with Atg7, Atg12-Atg5-Atg16, and liposomes generated from E. coli polar lipids as a source PE, and detected by migration of Atg8 in Coommassiestained SDS-PAGE gel. c, Effects of indicated mutants defective in a on Atg8 lipidation in vivo, as detected by western blot for Atg8 after 2 hours starvation of the XLY161 $\operatorname{atg} 3 \Delta$ pep $4 \Delta$ strain of S. cerevisiae expressing either WT or mutant HA-tagged Atg3. Dpm1 is loading control. 


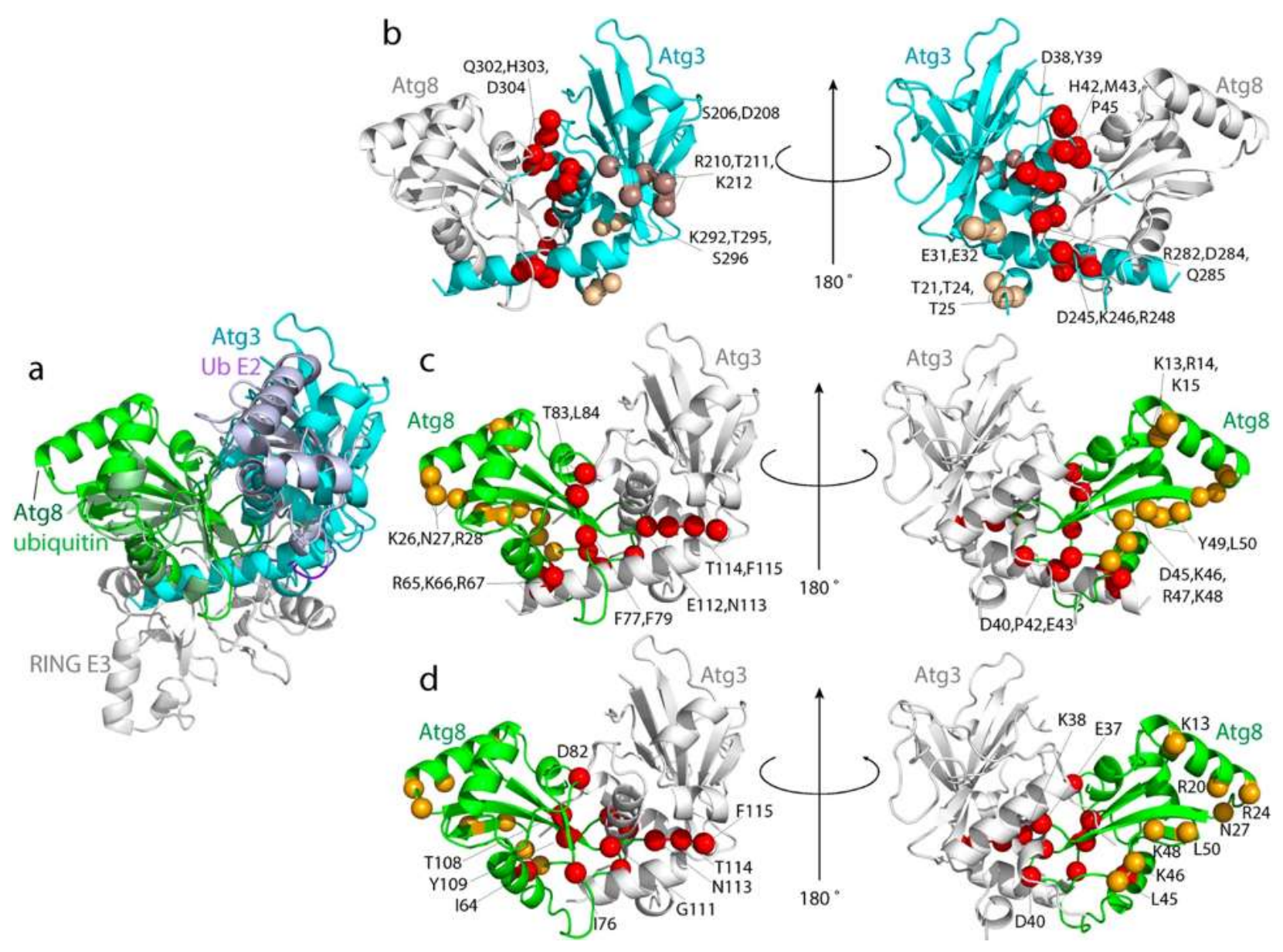

Figure 3-15. Modeling of the active conformation of Atg3 Atg8 intermediate.

a, Generation of model for a potential closed E2 Ubl conformation for Atg3 Atg8, with structures of $\operatorname{Atg} 3^{\triangle N F R}$ (PDB 6OJJ, from this study) and Atg8 (PDB 2ZPN) superimposed on E2 and Ub, respectively, in a RING E3-E2 Ub complex (PDB 4AP4). b, Sites of Atg3 mutations impairing E3-dependent activation of Atg3 Atg8, mapped on model for closed conformation. Red - residues corresponding to E2 Ub interface in closed conformation; wheat - residues corresponding to RING E3 binding site; bronze residues in catalytic segment. c, Sites of Atg8 mutations impairing E3-dependent activation of Atg3 Atg8, mapped on model for closed conformation. Red - residues corresponding to E2 $\mathrm{Ub}$ interface in closed conformation; orange - residues corresponding to AIM/LIR-binding site. d, Atg8 residues showing chemical shift perturbation one standard deviation above the mean upon covalent complex formation with Atg3, mapped on model for closed conformation. Red - residues corresponding to E2 Ub interface in closed conformation; orange - residues corresponding to AIM/LIRbinding site. 


\section{Data Availability}

The coordinates and structure factors for $\operatorname{Atg} 3^{\Delta \mathrm{NFR}}$ have been deposited to the RCSB Protein Data Bank with ID 6OJJ for immediate release upon manuscript

publication. The resonance assignments for Atg $3^{\text {cat }}, A \operatorname{tg} 3^{\mathrm{FR}}$ (residues 86-159), and Atg8 have been deposited to the Biological Magnetic Resonance Data Bank with the IDs 27922, 27923, and 27924, respectively for immediate release upon manuscript publication. 


\section{CHAPTER 4. DISCUSSION}

Here we demonstrated that Atg3 is restrained by an E123IR element within its 70residue flexible region bracing the active site; this brace establishes regulation by interacting with E1 (Figure 3-6a) (Taherbhoy et al., 2011), E2 (Figure 3-6c,d,f), and E3 (Figure 3-5a,c); and that a key function of the autophagy E3 Atg12-Atg5-Atg16 is to bind this brace to activate the intrinsic reactivity of the thioester-bonded Atg3 Atg8 intermediate (Figure 3-6b) (Zheng et al., 2019). This unprecedented allosteric mechanism underlying E2 activation - revealed by our NMR, crystallographic, and mutational data, taken together with prior studies - addresses the longstanding question of how Atg8 lipidation is activated by an E3 that lacks sequence and mechanistic conservation with canonical conjugation enzymes. Notably, E1-binding to the E123IR element is not sufficient to activate the Atg3 Atg8 intermediate (Figure 3-11), suggesting additional roles for E3 other than binding. Meanwhile, NMR data raise the possibility that Atg3's active site residues undergo conformational motions on a different time-scale from the rest of catalytic domain, which could impact enzymatic activity (Figure 3-6c). Taken together, we speculate that E3 binding to the Atg3 Atg8 intermediate broadly influences dynamic or structural features of Atg3's catalytic domain that in turn bias the active site in favor of the reactive conformation. Indeed, the need for E3-binding to activate the Atg3 Atg8 intermediate in vitro is partially ameliorated by mutations that map to the surfaces in and adjacent to the $\mathrm{Atg} 3^{\text {cat }}-\mathrm{Atg} 3^{\mathrm{E} 123 \mathrm{IR}}$ interface (Figure 3-10c,d and Figure 3-11). Physiological relevance is supported by such mutants also activating E3-independent lipidation in vivo (Figure 3-10e).

Although future studies will be required to visualize downstream steps in the reaction cascade, we propose that Atg8 lipidation involves Atg7, Atg3, and Atg12-Atg5Atg 16 toggling their common interacting region in Atg3 as follows: 1) Prior to encountering the $A \operatorname{tg} 7 \sim \operatorname{Atg} 8$ intermediate, the $A \operatorname{tg} 3^{\mathrm{E} 123 \mathrm{IR}}$ binds to the Atg3 catalytic domain to protect the catalytic Cys from access by non-specific cellular molecules. 2) The Atg7 Atg8 intermediate binds to Atg3's E123IR element using Atg7's N-terminal domain. This mediates both E1-E2 interactions and conformational activation of Atg3's catalytic Cys to receive Atg8 from Atg7. 3) Following formation of the Atg3 Atg8 intermediate, Atg3's E123IR element could re-engage the Atg3 catalytic domain to prevent Atg8 mis-ligation to a non-specific nucleophile. We speculate that this may also prevent the extreme $\mathrm{C}$-terminal residues of Atg3 from prematurely adopting a conformation that contributes to the activate conformation of the Atg3 A Atg8 intermediate. 4) With relatively higher binding affinity, the Atg12-Atg5 portion of the autophagy E3 subsequently dislodges Atg3's E123IR, which enables structural remodeling of the active site loop from a sequestered position to an exposed conformation where the catalytic Cys is juxtaposed with surrounding side-chains that are crucial for Atg8 lipidation (Figure 4-1). Apparently, Atg12-Atg5 further activates the lipidation reaction in a manner involving extensive surfaces from both Atg3 and Atg8 in the Atg3 Atg8 intermediate. In parallel with the mechanisms how RING and SUMO E3 activate canonical E2 UBL intermediates (Buetow and Huang, 2016; Cappadocia andLima, 2018; Zheng and Shabek, 2017), we speculate that the autophagy E3 


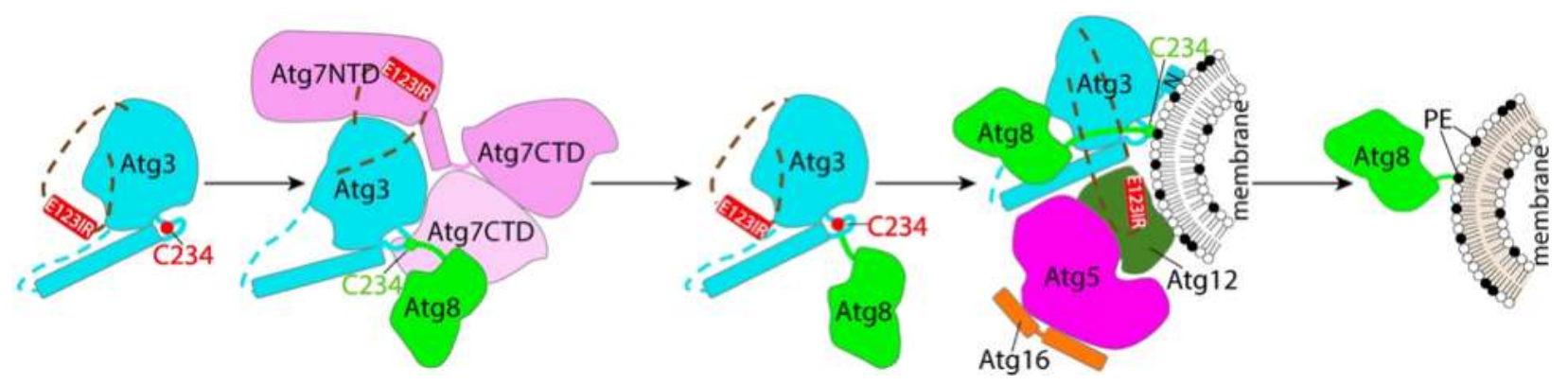

Figure 4-1. Schematic model for allosteric regulation of $\operatorname{Atg} 3$ activity through E123IR interactions across the lipidation cascade.

The autophagy E2 Atg3 is autoinhibited by Atg ${ }^{\text {E123IR }}$. When Atg3 encounters E1 Atg7, E123IR is relocated upon binding to $A \operatorname{tg} 7^{\mathrm{NTD}}$, thereby triggering rearrangement of the Atg3 catalytic core to activate Cys234 for attacking the Atg7 Atg8 intermediate. Atg8 is transferred from Atg7 to Atg8, producing the thioester-bonded Atg3 Atg8 intermediate. Relieved from Atg7, Atg $3^{\text {E123IR }}$ protects the Atg3 Atg8 intermediate from wayward discharge to errant nucleophiles. The E3 (Atg12-Atg5-Atg16) binds E123IR and further activates the Atg3 Atg8 intermediate for nucleophilic attack. Numerous interactions with membranes place this complex in proximity to PE for the lipidation reaction. 
Atg12-Atg5 mediates activation in part by promoting interactions between Atg3 and Atg8 in the Atg3 Atg8 intermediate, although details of the interaction remain elusive and might be distinct for the autophagy enzymes.

Why might the UBL conjugation pathway in autophagy involve such elaborate allosteric control? It seems likely that key features of the ultimate target, and intrinsic capabilities of the Atg3 Atg8 intermediate, impose distinctive requirements for regulation. First, the substrate PE is among the most abundant core glycerophospholipids. Found in several organelles, PE's widespread accessibility differs from canonical UB and UBL substrates, which typically only are licensed for modification after a regulatory event such as a post-translational modification directs E3 binding. Second, through its Nterminal membrane-curvature sensing helix, Atg3 itself can bind the substrate PE (Nath et al., 2014). The active site of Atg3 Atg8 may require protection to prevent inopportune discharge to PE, and this is best ensured by coming from the intermediate itself. Indeed, when conformationally activated by E3-mimicking mutations, Atg3 Atg8 can not only catalyze the lipidation reaction and in vivo, but also robustly discharges Atg8 to nonspecific nucleophiles even in the absence of E3. Thus, our data present a mechanism ensuring that Atg8 discharge is coupled to E3 binding, which in turn establishes targeting specificity through several features, including binding to cargo receptors (Fracchiolla et al., 2016), and to membranes (Lystad et al., 2019; Romanov et al., 2012). The Atg16 moiety in the autophagy E3 could subsequently engage the lipidated Atg8 to serve as a scaffold during autophagosome biogenesis (Kaufmann et al., 2014).

Allosteric E2 activation is emerging as a common property of E3 ligases (Buetow and Huang, 2016; Cappadocia and Lima, 2018; Zheng and Shabek, 2017). It is now wellrecognized that many E3 catalytic domains stabilize a "closed" conformation that renders E2 UB and E2 UBL intermediates susceptible to nucleophilic attack, while other E3 domains mediate substrate targeting. Many E2s are further activated allosterically, through their backside beta-sheets binding to noncatalytic E3 domains. These effects have largely been characterized for indirectly increasing binding to E3 RING domains. However, some E3 or E3-associated domains also trigger conformational changes at E2 active sites. For example, the membrane E3-associated Cue1p binds to Ubc7p's backside, and indirectly modulates the active site, tens of angstroms away (Metzger et al., 2013). Irrespective of whether E2 cysteines require protection from errant nucleophilic attack, or coordination of catalytic activation with other E3 functions, it seems likely that allosteric modulation of E2 active sites serves as a failsafe ensuring UB and UBL discharge at appropriate targets. The autophagy E2 Atg3 now serves as an ultimate case, with its brace self-imposed until directly lifted by the E3 to drive autophagy.

Apparently, lifting off the E123IR from Atg3 in the Atg3 Atg8 intermediate is not sufficient to fully activate Atg8-lipid conjugation, since adding E3 to WT Atg3 accelerates Atg8 lipidation more robustly than the auto-releasing Atg3 mutants in absence of E3. Moreover, our preliminary data from introducing additional E123IR peptides into in vitro constituted Atg8 lipidation system shows that E123IR is not sufficient to fully block the reaction. Here we suspect that other elements within the Atg3 FR may also help tuning the interactions between Atg3 Atg8 intermediate and Atg12- 
Atg5-Atg16. Our hypothesis can be tested by other research methods including solving the structure of the active conformation of Atg3 Atg8-Atg12-Atg5-Atg16 complex. However, since Atg3 Atg8 adopts multiple conformations shifting between active and inactive states, obtaining homogeneous in vitro recombinant protein samples of Atg3 Atg8-Atg12-Atg5-Atg16 can be exigent. Besides, since Atg3 contains several long flexible regions (e.g. FR and partial HR) that couldn't be removed due to their indispensable roles in enzyme function, it will be very challenging crystallizing the full complex. It is also potentially taxing to obtain cryo EM structures to display details of all the functional parts if they are largely unstructured.

As we discovered in this study, the C-terminus of Atg3 is associated with Atg8 when Atg3 Atg8 intermediate adopts the closed conformation, and its distinctive reversed AIM-like amino acid sequence of Met-Glu-Gly-Trp leads to the speculation that such interaction is realized by Atg8-AIM binding. In fact, interactions between Atg8 family protein and C-terminal LIR/AIM of other autophagic enzymes are not unpresented. The C-terminus of the autophay E1 Atg7 docks into the AIM-binding groove of its substrate Atg8, though it doesn't harbor a traditional AIM sequence (Noda et al., 2011). In human protein studies, the Phe-Glu-Ile-Leu sequence approaching the very end of ATG4 serves as a canonical LIR as binding to GABARAPL1, and was proved essential for ATG4's protease activity (Skytte Rasmussen et al., 2017). Intriguingly, such binding event similar to that between Atg8 and Atg3 C-terminus also takes place between canonical E2 and UBL. For example, the last few amino acids of yeast Cdc34 was proved directly binding to its substrate ubiquitin. Such interaction is functionally pronounced for Cdc34's catalytic activity (Choi et al., 2010).

According to our preliminary result of the Atg8 lipidation assay conducted inn S. cerevisiae in vivo cells, not all alanine mutagenesis introduced to the Atg3-binding sites in Atg8 showed decreased densities in lipidated Atg8 bands, while some mutants even harbor significantly higher lipidation levels. As we speculate, this inconsistency against the in vitro results using the same set of mutants (Figure 3-1) could be due to countering impacts from Atg4 against Atg3: for some Atg8 mutants, PE conjugation activity is impaired by defective Atg3-binding; however Atg4-mediated Atg8 cleavage from autophagic membrane is also diminished at the same time. Intriguingly and as expected, the Atg3- and Atg4-binding surfaces are largely overlapped on Atg8 if using human LC3ATG4B structure for comparison (Satoo et al., 2009). The results bring up a reasonable hypothesis that Atg8 binding to Atg3 and Atg4 is mutually exclusive, given Atg4 and Atg3 potentially play opposite roles in maintaining the total amount of lipidated Atg8 during autophagy progression. The unknown molecular requirements determining Atg8's preference between Atg4 and Atg3 indicate that Atg8-PE conjugation, as well as the whole autophagy flux, involves more complicated and delicate signaling pathways that need to be further studied.

Researchers now have limited knowledge about the membrane binding functions within enzymes in Atg8 lipidation cascade. Human protein study has shown that the Nterminal helix of Atg3 serves as membrane curvature detector which prefers binding to higher curvature liposome (Nath et al., 2014). Similar to other canonical E3 ligases that 
create physical proximity between E2 UBL intermediates and their specific substrates by binding to both, Atg12-Atg5-Atg16 also harbors membrane binding affinity in order to bring Atg3 Atg8 to substrate PE. According to the study in Saccharomyces Cerevisiae, membrane binding is mediated by Atg5, activated by Atg16, but inhibited by Atg12 (Romanov et al., 2012). Tested with in vitro lipid vesicles, this membrane binding feature enables the E3 complex to tether isolated membrane in absence of Atg8, and is also essential for autophagosome engulfment more than just for Atg8 lipidation (Romanov et al., 2012). However, the Atg5-lipid binding event takes place later than Atg12-Atg5Atg16 being located to the pre-autophagosomal structure (PAS) (Romanov et al., 2012), which suggests that the membrane system also utilizes other E3 recruiting mechanism besides Atg 5 interaction. In addition, recent studies reported multiple lipid-recognition domains across ATG16L1, including its N-terminal amphipathic helix, the C-terminal end, and the coiled-coil domain (Lystad et al., 2019). Notably, ATG16L1 harbors intrinsic lipid-binding that is independent from WIPI-mediated recruitment to PAS (Lystad et al., 2019). Multiple binding sites within the E2 and E3 complex, especially when they do not rely on one another or other lipid-binding machineries, indicates sophisticated regulatory mechanism orchestrating Atg8 family protein lipidation. The complicated nature of Atg12-Atg5-Atg16 interaction to PAS also hypothesizes that the roles of Atg12-Atg5-Atg16 in autophagy may be of greater variety than only to directly facilitate Atg8 transfer from Atg3 to PE.

According to current studies, inhibitors for autophagic proteins, especially for enzymes in Atg8 family protein lipidation cascade, are not being extensively investigated. Potential drug screenings against the lipidation enzymes may provide therapeutics to related diseases. Considering this, a great variety of distinctive mechanisms in the lipidation machinery can be targeted. Obviously, making proteinprotein interaction inhibitors is a reasonable idea to directly suppress LC3 lipidation activity, and to consequentially downregulate the entire autophagy level. Besides, cysteine interaction inhibitors could specifically attenuate the nucleophilic attacking efficiencies of autophagic E1 or E2, yet not interrupting E2-UBL, E2-substrate and E3E2 interactions. Nonetheless, our study also sheds light on the possibility to design Atg3 allosteric inhibitors, which allows more fine-tuned regulation by arresting Atg8 family protein lipidation in inactive states, and avoids disturbing any intrinsic enzyme activities. In summary, the dynamic nature of the Atg8 family protein lipidation system has now become an opportunity for drug discovery. 


\section{LIST OF REFERENCES}

Adams, P.D., Afonine, P.V., Bunkóczi, G., Chen, V.B., Davis, I.W., Echols, N., Headd, J.J., Hung, L.-W., Kapral, G.J., Grosse-Kunstleve, R.W., et al. (2010). PHENIX : a comprehensive Python-based system for macromolecular structure solution. Acta Crystallogr D Biol Crystallogr 66, 213-221.

Amaravadi, R., Kimmelman, A.C., and White, E. (2016). Recent insights into the function of autophagy in cancer. Genes Dev. 30, 1913-1930.

van Beek, N., Klionsky, D.J., and Reggiori, F. (2018). Genetic aberrations in macroautophagy genes leading to diseases. Biochimica et Biophysica Acta (BBA) Molecular Cell Research 1865, 803-816.

Berger, I., Fitzgerald, D.J., and Richmond, T.J. (2004). Baculovirus expression system for heterologous multiprotein complexes. Nature Biotechnology 22, 1583-1587.

Berndsen, C.E., Wiener, R., Yu, I.W., Ringel, A.E., and Wolberger, C. (2013). A conserved asparagine has a structural role in ubiquitin-conjugating enzymes. Nat Chem Biol 9, 154-156.

Birgisdottir, Å.B., Lamark, T., and Johansen, T. (2013). The LIR motif - crucial for selective autophagy. J Cell Sci 126, 3237-3247.

Bodemann, B.O., Orvedahl, A., Cheng, T., Ram, R.R., Ou, Y.-H., Formstecher, E., Maiti, M., Hazelett, C.C., Wauson, E.M., Balakireva, M., et al. (2011). RalB and the Exocyst Mediate the Cellular Starvation Response by Direct Activation of Autophagosome Assembly. Cell 144, 253-267.

Buetow, L., and Huang, D.T. (2016). Structural insights into the catalysis and regulation of E3 ubiquitin ligases. Nat Rev Mol Cell Biol 17, 626-642.

Cadwell, K., Patel, K.K., Komatsu, M., Herbert W. Virgin, I., and Stappenbeck, T.S. (2009). A common role for Atg16L1, Atg5, and Atg7 in small intestinal Paneth cells and Crohn disease. Autophagy 5, 250-252.

Cao, Y., Nair, U., Yasumura-Yorimitsu, K., and Klionsky, D.J. (2009). A multiple ATG gene knockout strain for yeast two-hybrid analysis. Autophagy 5, 699-705.

Cappadocia, L., and Lima, C.D. (2018). Ubiquitin-like Protein Conjugation: Structures, Chemistry, and Mechanism. Chem. Rev. 118, 889-918.

Carlos Martín Zoppino, F., Damián Militello, R., Slavin, I., Álvarez, C., and Colombo, M.I. (2010). Autophagosome Formation Depends on the Small GTPase Rab1 and Functional ER Exit Sites. Traffic 11, 1246-1261. 
Carlsson, S.R., and Simonsen, A. (2015). Membrane dynamics in autophagosome biogenesis. J Cell Sci 128, 193-205.

Chen, C.-Z., Ou, C.-Y., Wang, R.-H., Lee, C.-H., Lin, C.-C., Chang, H.-Y., and Hsiue, T.-R. (2015). Association of Egr-1 and autophagy-related gene polymorphism in men with chronic obstructive pulmonary disease. Journal of the Formosan Medical Association 114, 750-755.

Chen, D., Pang, S., Feng, X., Huang, W., Hawley, R.G., and Yan, B. (2013). Genetic analysis of the ATG7 gene promoter in sporadic Parkinson's disease. Neuroscience Letters 534, 193-198.

Cheng, Y., Ren, X., Hait, W.N., and Yang, J.-M. (2013). Therapeutic Targeting of Autophagy in Disease: Biology and Pharmacology. Pharmacol Rev 65, 1162-1197.

Choi, Y.-S., Wu, K., Jeong, K., Lee, D., Jeon, Y.H., Choi, B.-S., Pan, Z.-Q., Ryu, K.-S., and Cheong, C. (2010). The Human Cdc34 Carboxyl Terminus Contains a Non-covalent Ubiquitin Binding Activity That Contributes to SCF-dependent Ubiquitination. J. Biol. Chem. 285, 17754-17762.

Dikic, I. (2017). Proteasomal and Autophagic Degradation Systems. Annu. Rev. Biochem. 86, 193-224.

Dikic, I., and Elazar, Z. (2018). Mechanism and medical implications of mammalian autophagy. Nature Reviews Molecular Cell Biology 19, 349-364.

Emsley, P., Lohkamp, B., Scott, W.G., and Cowtan, K. (2010). Features and development of Coot. Acta Crystallogr D Biol Crystallogr 66, 486-501.

Feng, Y., He, D., Yao, Z., and Klionsky, D.J. (2014). The machinery of macroautophagy. Cell Research 24, 24-41.

Fracchiolla, D., Sawa-Makarska, J., Zens, B., Ruiter, A. de, Zaffagnini, G., Brezovich, A., Romanov, J., Runggatscher, K., Kraft, C., Zagrovic, B., et al. (2016). Mechanism of cargo-directed Atg8 conjugation during selective autophagy. ELife 5, e18544.

Fujita, N., Hayashi-Nishino, M., Fukumoto, H., Omori, H., Yamamoto, A., Noda, T., and Yoshimori, T. (2008a). An Atg4B Mutant Hampers the Lipidation of LC3 Paralogues and Causes Defects in Autophagosome Closure. MBoC 19, 4651-4659.

Fujita, N., Itoh, T., Omori, H., Fukuda, M., Noda, T., and Yoshimori, T. (2008b). The Atg16L Complex Specifies the Site of LC3 Lipidation for Membrane Biogenesis in Autophagy. MBoC 19, 2092-2100.

Galluzzi, L., Baehrecke, E.H., Ballabio, A., Boya, P., Bravo-San Pedro, J.M., Cecconi, F., Choi, A.M., Chu, C.T., Codogno, P., Colombo, M.I., et al. (2017). Molecular definitions of autophagy and related processes. The EMBO Journal 36, 1811-1836. 
Gatica, D., Lahiri, V., and Klionsky, D.J. (2018). Cargo recognition and degradation by selective autophagy. Nature Cell Biology 20, 233-242.

Gibson, D.G. (2011). Chapter fifteen - Enzymatic Assembly of Overlapping DNA Fragments. In Methods in Enzymology, C. Voigt, ed. (Academic Press), pp. 349-361.

Gibson, D.G., Young, L., Chuang, R.-Y., Venter, J.C., Hutchison, C.A., and Smith, H.O. (2009). Enzymatic assembly of DNA molecules up to several hundred kilobases. Nature Methods 6, 343-345.

Hanada, T., and Ohsumi, Y. (2005). Structure-Function Relationship of Atg12, a Ubiquitin-Like Modifier Essential for Autophagy. Autophagy 1, 110-118.

Hanada, T., Noda, N.N., Satomi, Y., Ichimura, Y., Fujioka, Y., Takao, T., Inagaki, F., and Ohsumi, Y. (2007). The Atg12-Atg5 Conjugate Has a Novel E3-like Activity for Protein Lipidation in Autophagy. J. Biol. Chem. 282, 37298-37302.

Harada, K., Kotani, T., Kirisako, H., Sakoh-Nakatogawa, M., Oikawa, Y., Kimura, Y., Hirano, H., Yamamoto, H., Ohsumi, Y., and Nakatogawa, H. (2019). Two distinct mechanisms target the autophagy-related E3 complex to the pre-autophagosomal structure. ELife 8, e43088.

Hikita, H., Sakane, S., and Takehara, T. (2018). Mechanisms of the autophagosomelysosome fusion step and its relation to non-alcoholic fatty liver disease. Liver Research $2,120-124$.

Hong, S.B., Kim, B.-W., Lee, K.-E., Kim, S.W., Jeon, H., Kim, J., and Song, H.K. (2011). Insights into noncanonical E1 enzyme activation from the structure of autophagic E1 Atg7 with Atg8. Nat Struct Mol Biol 18, 1323-1330.

Ichimura, Y., Kirisako, T., Takao, T., Satomi, Y., Shimonishi, Y., Ishihara, N., Mizushima, N., Tanida, I., Kominami, E., Ohsumi, M., et al. (2000). A ubiquitin-like system mediates protein lipidation. Nature 408, 488-492.

Kaiser, S.E., Qiu, Y., Coats, J.E., Mao, K., Klionsky, D.J., and Schulman, B.A. (2013). Structures of Atg7-Atg3 and Atg7-Atg10 reveal noncanonical mechanisms of E2 recruitment by the autophagy E1. Autophagy 9, 778-780.

Kaufmann, A., Beier, V., Franquelim, H.G., and Wollert, T. (2014). Molecular Mechanism of Autophagic Membrane-Scaffold Assembly and Disassembly. Cell 156, $469-481$.

Kim, J., Huang, W.-P., and Klionsky, D.J. (2001). Membrane Recruitment of Aut7p in the Autophagy and Cytoplasm to Vacuole Targeting Pathways Requires Aut1p, Aut2p, and the Autophagy Conjugation Complex. The Journal of Cell Biology 152, 51-64. 
Kim, M., Sandford, E., Gatica, D., Qiu, Y., Liu, X., Zheng, Y., Schulman, B.A., Xu, J., Semple, I., Ro, S.-H., et al. (2016). Mutation in ATG5 reduces autophagy and leads to ataxia with developmental delay. ELife 5, e12245.

Klionsky, D.J. (2000). Autophagy as a Regulated Pathway of Cellular Degradation. Science 290, 1717-1721.

Lane, J.D., Korolchuk, V.I., Murray, J.T., Zachari, M., and Ganley, I.G. (2017). The mammalian ULK1 complex and autophagy initiation. Essays Biochem 61, 585-596.

Lenz, H.D., Vierstra, R.D., Nürnberger, T., and Gust, A.A. (2011). ATG7 contributes to plant basal immunity towards fungal infection. Plant Signaling \& Behavior 6, 10401042.

Levy, J.M.M., Towers, C.G., and Thorburn, A. (2017). Targeting autophagy in cancer. Nature Reviews Cancer 17, 528-542.

Li, Q., Zhou, X., Huang, T., Tang, Y., Liu, B., Peng, P., Sun, L., Wang, Y., and Yuan, X. (2017a). The Thr300Ala variant of ATG16L1 is associated with decreased risk of brain metastasis in patients with non-small cell lung cancer. Autophagy 13, 1053-1063.

Li, Y., Huang, J., Pang, S., Wang, H., Zhang, A., Hawley, R.G., and Yan, B. (2017b). Novel and functional ATG12 gene variants in sporadic Parkinson's disease.

Neuroscience Letters 643, 22-26.

Longatti, A., Lamb, C.A., Razi, M., Yoshimura, S., Barr, F.A., and Tooze, S.A. (2012). TBC1D14 regulates autophagosome formation via Rab11- and ULK1-positive recycling endosomes. The Journal of Cell Biology 197, 659-675.

Lyamzaev, K.G., Tokarchuk, A.V., Panteleeva, A.A., Mulkidjanian, A.Y., Skulachev, V.P., and Chernyak, B.V. (2018). Induction of autophagy by depolarization of mitochondria. Autophagy 14, 921-924.

Lystad, A.H., Carlsson, S.R., de la Ballina, L.R., Kauffman, K.J., Nag, S., Yoshimori, T., Melia, T.J., and Simonsen, A. (2019). Distinct functions of ATG16L1 isoforms in membrane binding and LC3B lipidation in autophagy-related processes. Nat Cell Biol 21, $372-383$.

Marsh, J.A., Singh, V.K., Jia, Z., and Forman-Kay, J.D. (2006). Sensitivity of secondary structure propensities to sequence differences between $\alpha$ - and $\gamma$-synuclein: Implications for fibrillation. Protein Sci. 15, 2795-2804.

Martin, L.J., Gupta, J., Jyothula, S.S.S.K., Butsch Kovacic, M., Biagini Myers, J.M., Patterson, T.L., Ericksen, M.B., He, H., Gibson, A.M., Baye, T.M., et al. (2012). Functional Variant in the Autophagy-Related 5 Gene Promotor is Associated with Childhood Asthma. PLoS ONE 7, e33454. 
Maruyama, T., and Noda, N.N. (2018). Autophagy-regulating protease Atg4: structure, function, regulation and inhibition. The Journal of Antibiotics 71, 72-78.

Metlagel, Z., Otomo, C., Takaesu, G., and Otomo, T. (2013). Structural basis of ATG3 recognition by the autophagic ubiquitin-like protein ATG12. PNAS 110, 18844-18849.

Metlagel, Z., Otomo, C., Ohashi, K., Takaesu, G., and Otomo, T. (2014). Structural insights into E2-E3 interaction for LC3 lipidation. Autophagy 10, 522-523.

Metzger, M.B., Liang, Y.-H., Das, R., Mariano, J., Li, S., Li, J., Kostova, Z., Byrd, R.A., Ji, X., and Weissman, A.M. (2013). A Structurally Unique E2-Binding Domain Activates Ubiquitination by the ERAD E2, Ubc7p, through Multiple Mechanisms. Molecular Cell $50,516-527$.

Metzger, S., Saukko, M., Van Che, H., Tong, L., Puder, Y., Riess, O., and Nguyen, H.P. (2010). Age at onset in Huntington's disease is modified by the autophagy pathway: implication of the V471A polymorphism in Atg7. Hum Genet 128, 453-459.

Mizushima, N. (2007). Autophagy: process and function. Genes Dev. 21, 2861-2873.

Mizushima, N., Noda, T., and Ohsumi, Y. (1999). Apg16p is required for the function of the Apg12p-Apg5p conjugate in the yeast autophagy pathway. EMBO J 18, 3888-3896.

Mizushima, N., Levine, B., Cuervo, A.M., and Klionsky, D.J. (2008). Autophagy fights disease through cellular self-digestion. NATURE 451, 1069-1075.

Moloughney, J.G., Monken, C.E., Tao, H., Zhang, H., Thomas, J.D., Lattime, E.C., and Jin, S.V. (2011). Vaccinia virus leads to ATG12-ATG3 conjugation and deficiency in autophagosome formation. Autophagy 7, 1434-1447.

Murshudov, G.N., Vagin, A.A., and Dodson, E.J. (1997). Refinement of Macromolecular Structures by the Maximum-Likelihood Method. Acta Crystallogr D Biol Crystallogr 53, 240-255.

Nakatogawa, H., Ichimura, Y., and Ohsumi, Y. (2007). Atg8, a Ubiquitin-like Protein Required for Autophagosome Formation, Mediates Membrane Tethering and Hemifusion. Cell 130, 165-178.

Nakatogawa, H., Suzuki, K., Kamada, Y., and Ohsumi, Y. (2009). Dynamics and diversity in autophagy mechanisms: lessons from yeast. Nature Reviews Molecular Cell Biology 10, 458-467.

Nath, S., Dancourt, J., Shteyn, V., Puente, G., Fong, W.M., Nag, S., Bewersdorf, J., Yamamoto, A., Antonny, B., and Melia, T.J. (2014). Lipidation of the LC3/GABARAP family of autophagy proteins relies on a membrane-curvature-sensing domain in Atg3. Nat Cell Biol 16, 415-424. 
Noda, T., and Klionsky, D.J. (2008). Chapter 3 The Quantitative Pho8 860 Assay of Nonspecific Autophagy. In Methods in Enzymology, (Elsevier), pp. 33-42.

Noda, N.N., Ohsumi, Y., and Inagaki, F. (2010). Atg8-family interacting motif crucial for selective autophagy. FEBS Letters 584, 1379-1385.

Noda, N.N., Satoo, K., Fujioka, Y., Kumeta, H., Ogura, K., Nakatogawa, H., Ohsumi, Y., and Inagaki, F. (2011). Structural Basis of Atg8 Activation by a Homodimeric E1, Atg7. Molecular Cell 44, 462-475.

Noda, N.N., Fujioka, Y., Hanada, T., Ohsumi, Y., and Inagaki, F. (2013). Structure of the Atg12-Atg5 conjugate reveals a platform for stimulating Atg8-PE conjugation. EMBO Reports 14, 206-211.

Ohashi, K., and Otomo, T. (2015). Identification and characterization of the linear region of ATG3 that interacts with ATG7 in higher eukaryotes. Biochemical and Biophysical Research Communications 463, 447-452.

Ohsumi, Y., and Mizushima, N. (2004). Two ubiquitin-like conjugation systems essential for autophagy. Seminars in Cell \& Developmental Biology 15, 231-236.

Otomo, C., Metlagel, Z., Takaesu, G., and Otomo, T. (2013). Structure of the human ATG12 ATG5 conjugate required for LC3 lipidation in autophagy. Nature Structural \& Molecular Biology 20, 59-66.

Proikas-Cezanne, T., Takacs, Z., Donnes, P., and Kohlbacher, O. (2015). WIPI proteins: essential PtdIns3P effectors at the nascent autophagosome. Journal of Cell Science 128, 207-217.

Puri, P., and Chandra, A. (2014). Autophagy Modulation As a Potential Therapeutic Target for Liver Diseases. Journal of Clinical and Experimental Hepatology 4, 51-59.

Puri, C., Renna, M., Bento, C.F., Moreau, K., and Rubinsztein, D.C. (2013). Diverse Autophagosome Membrane Sources Coalesce in Recycling Endosomes. Cell 154, 12851299.

Qi, Y., Zhou, X., Nath, S.K., Sun, C., Wang, Y., Hou, P., Mu, R., Li, C., Guo, J., Li, Z., et al. (2018). A Rare Variant (rs933717) at FBXO31-MAP1LC3B in Chinese Is Associated With Systemic Lupus Erythematosus. Arthritis Rheumatol 70, 287-297.

Qiu, Y., Hofmann, K., Coats, J.E., Schulman, B.A., and Kaiser, S.E. (2013). Binding to E1 and E3 is mutually exclusive for the human autophagy E2 Atg3. Protein Science 22, 1691-1697.

Rambold, A.S., and Lippincott-Schwartz, J. (2011). Mechanisms of mitochondria and autophagy crosstalk. Cell Cycle 10, 4032-4038. 
Ravikumar, B., Moreau, K., Jahreiss, L., Puri, C., and Rubinsztein, D.C. (2010). Plasma membrane contributes to the formation of pre-autophagosomal structures. Nat Cell Biol $12,747-757$.

Reed, M., Morris, S.H., Owczarczyk, A.B., and Lukacs, N.W. (2015). Deficiency of autophagy protein Map1-LC3b mediates IL-17-dependent lung pathology during respiratory viral infection via ER stress-associated IL-1. Mucosal Immunology 8, 11181130.

Rogov, V., Dötsch, V., Johansen, T., and Kirkin, V. (2014). Interactions between Autophagy Receptors and Ubiquitin-like Proteins Form the Molecular Basis for Selective Autophagy. Molecular Cell 53, 167-178.

Romanov, J., Walczak, M., Ibiricu, I., Schüchner, S., Ogris, E., Kraft, C., and Martens, S. (2012). Mechanism and functions of membrane binding by the Atg5-Atg12/Atg16 complex during autophagosome formation: Role of Atg5-Atg12/Atg16 during autophagy. The EMBO Journal 31, 4304-4317.

Rubinsztein, D.C., Shpilka, T., and Elazar, Z. (2012). Mechanisms of Autophagosome Biogenesis. Current Biology 22, R29-R34.

Saha, A., and Deshaies, R.J. (2008). Multimodal Activation of the Ubiquitin Ligase SCF by Nedd8 Conjugation. Molecular Cell 32, 21-31.

Sakoh-Nakatogawa, M., Matoba, K., Asai, E., Kirisako, H., Ishii, J., Noda, N.N., Inagaki, F., Nakatogawa, H., and Ohsumi, Y. (2013). Atg12-Atg5 conjugate enhances E2 activity of Atg3 by rearranging its catalytic site. Nature Structural \& Molecular Biology 20, 433439.

Satoo, K., Noda, N.N., Kumeta, H., Fujioka, Y., Mizushima, N., Ohsumi, Y., and Inagaki, F. (2009). The structure of Atg4B-LC3 complex reveals the mechanism of LC3 processing and delipidation during autophagy. EMBO J 28, 1341-1350.

Shao, Y., Chen, F., Chen, Y., Zhang, W., Lin, Y., Cai, Y., Yin, Z., Tao, S., Liao, Q., Zhao, J., et al. (2017). Association between genetic polymorphisms in the autophagyrelated 5 gene promoter and the risk of sepsis. Scientific Reports 7, 1-14.

Shen, M., and Lin, L. (2019). Functional variants of autophagy-related genes are associated with the development of hepatocellular carcinoma. Life Sciences 235, 116675.

Shpilka, T., Weidberg, H., Pietrokovski, S., and Elazar, Z. (2011). Atg8: an autophagyrelated ubiquitin-like protein family. Genome Biology 12, 226.

Shpilka, T., Mizushima, N., and Elazar, Z. (2012). Ubiquitin-like proteins and autophagy at a glance. J Cell Sci 125, 2343-2348.

Skytte Rasmussen, M., Mouilleron, S., Kumar Shrestha, B., Wirth, M., Lee, R., Bowitz Larsen, K., Abudu Princely, Y., O’Reilly, N., Sjøttem, E., Tooze, S.A., et al. (2017). 
ATG4B contains a C-terminal LIR motif important for binding and efficient cleavage of mammalian orthologs of yeast Atg8. Autophagy 13, 834-853.

Sou, Y., Tanida, I., Komatsu, M., Ueno, T., and Kominami, E. (2006).

Phosphatidylserine in Addition to Phosphatidylethanolamine Is an in Vitro Target of the Mammalian Atg8 Modifiers, LC3, GABARAP, and GATE-16. J. Biol. Chem. 281, 3017-3024.

Stolz, A., Ernst, A., and Dikic, I. (2014). Cargo recognition and trafficking in selective autophagy. Nature Cell Biology 16, 495-501.

Suzuki, K. (2001). The pre-autophagosomal structure organized by concerted functions of APG genes is essential for autophagosome formation. The EMBO Journal 20, 59715981.

Taherbhoy, A.M., Tait, S.W., Kaiser, S.E., Williams, A.H., Deng, A., Nourse, A., Hammel, M., Kurinov, I., Rock, C.O., Green, D.R., et al. (2011). Atg8 Transfer from Atg7 to Atg3: A Distinctive E1-E2 Architecture and Mechanism in the Autophagy Pathway. Molecular Cell 44, 451-461.

Tanida, I., Mizushima, N., Kiyooka, M., Ohsumi, M., Ueno, T., Ohsumi, Y., and Kominami, E. (1999). Apg7p/Cvt2p: A Novel Protein-activating Enzyme Essential for Autophagy. MBoC 10, 1367-1379.

Tanida, I., Sou, Y., Ezaki, J., Minematsu-Ikeguchi, N., Ueno, T., and Kominami, E. (2004a). HsAtg4B/HsApg4B/Autophagin-1 Cleaves the Carboxyl Termini of Three Human Atg8 Homologues and Delipidates Microtubule-associated Protein Light Chain 3and GABAA Receptor-associated Protein-Phospholipid Conjugates. J. Biol. Chem. 279, $36268-36276$.

Tanida, I., Ueno, T., and Kominami, E. (2004b). Human Light Chain 3/MAP1LC3B Is Cleaved at Its Carboxyl-terminal Met ${ }^{121}$ to Expose Gly ${ }^{120}$ for Lipidation and Targeting to Autophagosomal Membranes. J. Biol. Chem. 279, 47704-47710.

Tanida, I., Ueno, T., and Kominami, E. (2004c). LC3 conjugation system in mammalian autophagy. The International Journal of Biochemistry \& Cell Biology 36, 2503-2518.

Tanida, I., Ueno, T., and Kominami, E. (2008). LC3 and Autophagy. In Autophagosome and Phagosome, V. Deretic, ed. (Totowa, NJ: Humana Press), pp. 77-88.

Tindwa, H., Jo, Y.H., Patnaik, B.B., Noh, M.Y., Kim, D.H., Kim, I., Han, Y.S., Lee, Y.S., Lee, B.L., and Kim, N.J. (2015). DEPLETION OF AUTOPHAGY-RELATED GENES ATG3 AND ATG5 IN Tenebrio molitor LEADS TO DECREASED SURVIVABILITY AGAINST AN INTRACELLULAR PATHOGEN, Listeria monocytogenes. Archives of Insect Biochemistry and Physiology 88, 85-99. 
Tsuboyama, K., Koyama-Honda, I., Sakamaki, Y., Koike, M., Morishita, H., and Mizushima, N. (2016). The ATG conjugation systems are important for degradation of the inner autophagosomal membrane. Science 354, 1036-1041.

Usategui-Martín, R., García-Aparicio, J., Corral-Gudino, L., Calero-Paniagua, I., Del Pino-Montes, J., and González Sarmiento, R. (2015). Polymorphisms in Autophagy Genes Are Associated with Paget Disease of Bone. PLoS ONE 10, e0128984.

van der Vaart, A., Griffith, J., and Reggiori, F. (2010). Exit from the Golgi Is Required for the Expansion of the Autophagosomal Phagophore in Yeast Saccharomyces cerevisiae. MBoC 21, 2270-2284.

Walczak, M., and Martens, S. (2013). Dissecting the role of the Atg12-Atg5-Atg16 complex during autophagosome formation. Autophagy 9, 424-425.

Weissmann, F., Petzold, G., VanderLinden, R., Huis in 't Veld, P.J., Brown, N.G., Lampert, F., Westermann, S., Stark, H., Schulman, B.A., and Peters, J.-M. (2016). biGBac enables rapid gene assembly for the expression of large multisubunit protein complexes. Proc Natl Acad Sci USA 113, E2564-E2569.

Wild, P., McEwan, D.G., and Dikic, I. (2014). The LC3 interactome at a glance. J Cell Sci 127, 3-9.

Wileman, T. (2013). Autophagy as a defence against intracellular pathogens. Essays in Biochemistry 55, 153-163.

Wu, D.-H., Jia, C.-C., Chen, J., Lin, Z.-X., Ruan, D.-Y., Li, X., Lin, Q., Min-Dong, Ma, X.-K., Wan, X.-B., et al. (2014). Autophagic LC3B overexpression correlates with malignant progression and predicts a poor prognosis in hepatocellular carcinoma. Tumor Biol. 35, 12225-12233.

Xue, L., Chiu, S., and Oleinick, N.L. (2010). Atg7 deficiency increases resistance of MCF-7 human breast cancer cells to photodynamic therapy. Autophagy 6, 248-255.

Yamada, Y., Suzuki, N.N., Hanada, T., Ichimura, Y., Kumeta, H., Fujioka, Y., Ohsumi, Y., and Inagaki, F. (2007). The Crystal Structure of Atg3, an Autophagy-related Ubiquitin Carrier Protein (E2) Enzyme that Mediates Atg8 Lipidation. J. Biol. Chem. 282, 8036-8043.

Yamaguchi, M., Noda, N.N., Nakatogawa, H., Kumeta, H., Ohsumi, Y., and Inagaki, F. (2010). Autophagy-related Protein 8 (Atg8) Family Interacting Motif in Atg3 Mediates the Atg3-Atg8 Interaction and Is Crucial for the Cytoplasm-to-Vacuole Targeting Pathway. J. Biol. Chem. 285, 29599-29607.

Yamaguchi, M., Matoba, K., Sawada, R., Fujioka, Y., Nakatogawa, H., Yamamoto, H., Kobashigawa, Y., Hoshida, H., Akada, R., Ohsumi, Y., et al. (2012). Noncanonical recognition and UBL loading of distinct E2s by autophagy-essential Atg7. Nat Struct Mol Biol 19, 1250-1256. 
Yang, Z., and Klionsky, D.J. (2010). Eaten alive: a history of macroautophagy. Nature Cell Biology 12, 814-822.

Zaffagnini, G., and Martens, S. (2016). Mechanisms of Selective Autophagy. Journal of Molecular Biology 428, 1714-1724.

Zheng, N., and Shabek, N. (2017). Ubiquitin Ligases: Structure, Function, and Regulation. Annu. Rev. Biochem. 86, 129-157.

Zheng, M., Yu, H., Zhang, L., Li, H., Liu, Y., Kijlstra, A., and Yang, P. (2015). Association of ATG5 Gene Polymorphisms With Behçet's Disease and ATG10 Gene Polymorphisms With VKH Syndrome in a Chinese Han Population. Invest. Ophthalmol. Vis. Sci. 56, 8280 .

Zheng, Y., Qiu, Y., Gunderson, J.E.C., and Schulman, B.A. (2017). Production of Human ATG Proteins for Lipidation Assays. In Methods in Enzymology, (Elsevier), pp. 97-113.

Zheng, Y., Qiu, Y., Grace, C.R.R., Liu, X., Klionsky, D.J., and Schulman, B.A. (2019). A switch element in the autophagy E2 Atg3 mediates allosteric regulation across the lipidation cascade. Nature Communications 10, 1-14.

Zhou, X. -j., Lu, X. -1., Lv, J. -c., Yang, H. -z., Qin, L. -x., Zhao, M. -h., Su, Y., Li, Z. -g., and Zhang, H. (2011). Genetic association of PRDM1-ATG5 intergenic region and autophagy with systemic lupus erythematosus in a Chinese population. Annals of the Rheumatic Diseases 70, 1330-1337. 


\section{VITA}

Yumei Zheng was born in Wuhan, China in 1990. She received her Bachelor of Science from Huazhong University of Science and Technology in Wuhan, China, with a major in Biotechnology. She moved to United States and started her doctoral study in the Integrated Biomedical Sciences Program at University of Tennessee Health Science Center in 2013. She is expected to receive her Ph.D. degree in May of 2020. 